\title{
Machine Learning for Biomarker Discovery: Significant Pattern
} Mining

\section{Book Chapter}

Author(s):

Llinares-López, Felipe; Borgwardt, Karsten

Publication date:

2019

Permanent link:

https://doi.org/10.3929/ethz-b-000390151

\section{Rights / license:}

Creative Commons Attribution-NonCommercial-NoDerivatives 4.0 International

Originally published in:

https://doi.org/10.1017/9781108377706.009

\section{Funding acknowledgement:}

316861 - Machine Learning for Personalized Medicine (EC)

155913 - Significant Pattern Mining (SNF) 


\section{Machine Learning for Biomarker Discovery: Significant Pattern Mining}

\section{Felipe Llinares-López and Karsten Borgwardt}

\subsection{Introduction}

Biomarker discovery, the search for measurable biological indicators of a phenotypic trait of interest, is one of the fundamental data analysis problems in computational biology and healthcare. The challenging nature of datasets in those fields, typically containing many more features than samples, has motivated the development of novel tools for statistical inference in high-dimensional spaces. An example of such datasets may contain gene expression profiles of hundreds of individuals (samples), in which tens of thousands of genes (features) are surveyed for each individual. In this example, the goal would be to identify the biomarkers (e.g., genes) that help us differentiate between individuals of distinct phenotypes. Existing work has predominantly focused on either univariate feature selection methods (e.g., [1]), which consider the effect of each candidate marker in isolation from the others, or multivariate linear models with sparsity-inducing regularisers (e.g., $[2,3,4,5,6,7]$ ), which jointly model the effect of all candidate markers as a weighted additive combination of individual effects. The application of these techniques in biology and medicine has led to many successes. For instance, they have been extensively used in genome-wide association studies, leading to the discovery of more than 30,000 variant-trait associations [8], many of which led to substantial biological insight and even clinical applications [9]. However, a common limitation of both families of approaches is their inability to discover nonlinear signals due to interactions between features. For instance, this "blind spot" has been hypothesized as a factor that could account for at least a fraction of the "missing heritability" in genome-wide association studies [10, 11, 12, 13], that is, the phenomenon that loci discovered by genome-wide association studies only account for a small proportion of the estimated heritability of the phenotypes.

However, assessing the statistical association of all high-order feature interactions with a phenotypic trait of interest is a difficult problem. By explicitly considering all 
high-order feature interactions, the effective number of features in the model explodes combinatorially, further exacerbating the gap between sample size and number of features. To give a sense of scale, in a dataset with $p=266$ features, which could be considered small by current standards, one could enumerate up to $2^{p} \approx 10^{80}$ high-order feature interactions; as many as the estimated number of electrons in the observable universe [14, appendix C.4]. The enormous number of feature interactions that would need to be tested for association with the trait of interest creates two fundamental challenges:

1. A statistical challenge, caused by the need to perform statistical inference in the extremely high-dimensional space resulting from explicitly considering all high-order feature interactions. This is the so called multiple hypothesis testing problem, a phenomenon that makes it considerably challenging to control the probability to report false associations while maintaining enough statistical power to discover the truly significant high-order feature interactions.

2. A computational challenge, derived from the need to explore the vast search space consisting of all candidate feature interactions.

Significant pattern mining is a novel branch of machine learning developed specifically to tackle these challenges $[15,16,17,18,19,20]$. The goal of this chapter is to provide readers with the necessary tools to understand state-of-the-art significant pattern mining approaches and to apply these algorithms in data analysis tasks in their respective fields of research. The rest of this chapter is organised as follows:

Section 8.2 is devoted to introduce, in a self-contained manner, the key background concepts the rest of this chapter relies on. Section 8.2.1 defines a general framework for significant pattern mining and details two of its most common instances: (i) significant itemset mining, which aims at finding significant high-order interactions between binary features, and ii) significant subgraph mining, which looks for significant subgraphs among a database of graph-structured samples. Sections 8.2.2 and 8.2.3 deal with the statistical aspects of significant pattern mining. Section 8.2.2 provides background on statistical association testing for binary random variables, one of the key sub-components of significant pattern mining. Section 8.2.3 discusses the multiple hypothesis testing problem, the main statistical difficulty that significant pattern mining faces, and presents Tarone's improved Bonferroni correction for discrete data. This is the key statistical tool that significant pattern mining algorithms use to solve that difficulty.

Section 8.3 builds upon previous sections and details how to combine Tarone's improved Bonferroni correction with classical data mining techniques, leading to an efficient approach to analyse large databases using Tarone's method.

Sections 8.4 and 8.5 present recent advances in significant pattern mining: Section 8.4 shows how the inherent redundancy between candidate feature interactions can be exploited to gain additional statistical power and Section 8.5 describes how confounding factors such as age, gender, or population structure can be accounted for in significant pattern mining.

Finally, Section 8.6 synthesizes the main concepts of this chapter, gives pointers to existing software and discusses the main open problems in the field. 


\subsection{The Problem of Significant Pattern Mining}

\subsubsection{Terminology and Problem Statement}

Suppose we are given a dataset $\mathcal{D}=\left\{\left(x_{i}, y_{i}\right)\right\}_{i=1}^{n}$ consisting of $n$ independent and identically distributed (i.i.d.) samples $x$ taking values in an input domain $\mathcal{X}$ and assigned to one of two classes $y \in\{0,1\}$. Informally, we call any discrete substructure of the input samples $x \in \mathcal{X}$ a pattern.

The goal of significant pattern mining is to find, given a search space $\mathcal{M}$ containing candidate patterns of interest, all patterns $\mathcal{S} \in \mathcal{M}$ that occur statistically significantly more often in one class of samples than in the other.

Let the pattern occurrence indicator $g_{\mathcal{S}}(x)$ be defined as the binary random variable that indicates whether pattern $\mathcal{S}$ occurs in an input sample $x$ or not:

$$
g_{\mathcal{S}}(x)= \begin{cases}1, & \text { if } \mathcal{S} \subseteq x \\ 0, & \text { otherwise }\end{cases}
$$

Then, determining if pattern $\mathcal{S}$ is statistically significantly enriched in input samples $x$ belonging to one of the two classes is equivalent to testing the statistical association of two binary random variables, the class labels $y$ and the indicator $g_{\mathcal{S}}(x)$, based on $n$ observations $\left\{\left(g_{\mathcal{S}}\left(x_{i}\right), y_{i}\right)\right\}_{i=1}^{n}$ from $\mathcal{D}$.

The precise definition of a pattern $\mathcal{S}$, the corresponding search space of candidate patterns $\mathcal{M}$ and the concept of inclusion $\mathcal{S} \subseteq x$ all depend on the nature on the input domain $\mathcal{X}$. This leads to multiple instances of significant pattern mining, all of which fit in the same abstract framework described above.

\subsubsection{Significant Itemset Mining}

One of the most common instances of significant pattern mining arises when the input samples are $p$-dimensional binary vectors, i.e., $\mathcal{X}=\{0,1\}^{p}$. Conceptually, this means that each input sample $x$ is being represented by $p$ distinct features $x=\left(u_{1}, u_{2}, \ldots, u_{p}\right)$, each of which can be either active $\left(u_{j}=1\right)$ or inactive $\left(u_{j}=0\right)$.

This type of representation can be readily applied to a wide variety of applications of interest to computational biology and clinical data analysis. For instance, in a genome-wide association study, each of these $p$ binary features could be the result of a dominant/recessive/over-dominant encoding of single nucleotide polymorphisms (SNPs) or be obtained based on prior knowledge such as functional annotations. In functional genomics, each of the $n$ input samples could correspond to a different genomic region while each of the $p$ binary features could indicate whether a certain property applies to the genomic region or not, such as containing a specific transcription factor binding motif or exhibiting a particular chromatin modification in a given cell type. As another example, in a clinical setting, this type of representation could be used to describe the results of laboratory tests for a set of patients, with each of the $p$ binary features encoding the result of a certain test as either abnormal or normal. Electronic health records (EHRs) can also be partly represented in this form, with each of the $p$ binary features indicating which medical codes from a certain medical ontology (e.g., ICD-9, SNOMED-CT) apply to the record. 
In significant itemset mining, each pattern $\mathcal{S}$ corresponds to a specific high-order feature interaction. Let $\mathcal{S} \subseteq\{1,2, \ldots, p\}$ index an arbitrary subset of the $p$ binary features. Define the high-order interaction between the features indexed by $\mathcal{S}$ as $z_{\mathcal{S}}(x)=$ $\prod_{j \in \mathcal{S}} u_{j}$. Note that $z_{\mathcal{S}}(x)=1$ if and only if all features in $\mathcal{S}$ are simultaneously active in the input sample $x$ and $z_{\mathcal{S}}(x)=0$ otherwise. We define a pattern $\mathcal{S}$ to occur in a sample $x \in\{0,1\}^{p}$, i.e., $\mathcal{S} \subseteq x$, if the high-order feature interaction induced by $\mathcal{S}$ is active $\left(z_{\mathcal{S}}(x)=1\right)$. In other words, $g_{\mathcal{S}}(x)=z_{\mathcal{S}}(x)$. This definition can be justified as follows. Suppose each input sample $x \in\{0,1\}^{p}$ is alternatively represented as the set containing the indices of active features in $x$. For example, with $p=5$, we can represent an input sample $x_{1}=(1,0,1,1,0)$ as $x_{1}=\{1,3,4\}$ while $x_{2}=(0,1,0,1,0)$ can be represented as $x_{2}=\{2,4\}$. Since this is a one-to-one mapping between the set of $p$-dimensional binary vectors $\{0,1\}^{p}$ and the set of all subsets of $\{1,2, \ldots, p\}$, denoted the powerset $\mathcal{P}(\{1,2, \ldots, p\})$, both representations are equivalent and the input domain can also be defined as $\mathcal{X}=\mathcal{P}(\{1,2, \ldots, p\})$. However, we have $\mathcal{S} \subseteq x$, with $x$ represented as a set of active features and inclusion defined in the traditional sense, if and only if the high-order feature interaction $z_{\mathcal{S}}(x)$ as defined above takes value 1. Hence, both representations lead to the same pattern occurrence indicator $g_{\mathcal{S}}(x)$. This notation, which represents input samples as a set of active features or items, is widespread in the data mining field. This is primarily the reason why this particular instance of significant pattern mining is commonly referred to as significant itemset mining.

In applications in which all feature interactions are of potential interest, the search space of candidate patterns $\mathcal{M}$ would contain all possible feature subsets, i.e., $\mathcal{M}=\mathcal{P}(\{1,2, \ldots, p\})$. Thus, the search space would contain $|\mathcal{M}|=2^{p}$ patterns. ${ }^{1}$ While this is the most common case, one can incorporate certain forms of prior knowledge by explicitly restricting the feature interactions contained in $\mathcal{M}$. For example, in the context of a genome-wide association study, a researcher might wish to focus the analysis on feature interactions occurring between variants belonging to the same gene or between variants belonging to the same biological pathway. This flexibility to encode prior knowledge leads to a reduction in the number of candidate patterns, thus improving the statistical power and reducing runtime. It also opens the door to the development of novel variations of significant itemset mining, targeting specific applications in computational biology and medicine.

Figure 8.1 illustrates a canonical application of significant itemset mining to a toy dataset containing $n=12$ samples, $n_{1}=6$ of which belong to class $y=1$ (e.g., cases) and $n_{0}=6$ of which belong to class $y=0$ (e.g., controls). Each sample is represented by a binary vector with $p=10$ features, $u_{1}, u_{2}, \ldots, u_{10}$. Two patterns, $\mathcal{S}_{1}=\{1,3,5,6\}$ (yellow) and $\mathcal{S}_{2}=\{2,9,10\}$ (blue), and their respective high-order feature interactions, $z_{\mathcal{S}_{1}}(x)$ and $z_{\mathcal{S}_{2}}(x)$, are highlighted in the figure. In this example, none of the 10 features is individually associated with the class labels. Therefore, a univariate analysis or a multivariate linear model would not capture any association. However, visual inspection reveals that the high-order feature interactions $z_{\mathcal{S}_{1}}(x)$ and $z_{\mathcal{S}_{2}}(x)$ are active considerably more often for samples in class $y=1$ than for samples in class $y=0$. The fact that significant high-order interactions can be present

1 We denote by $|\mathcal{M}|$ the cardinality of a set $\mathcal{M}$, that is, the number of elements in $\mathcal{M}$. 


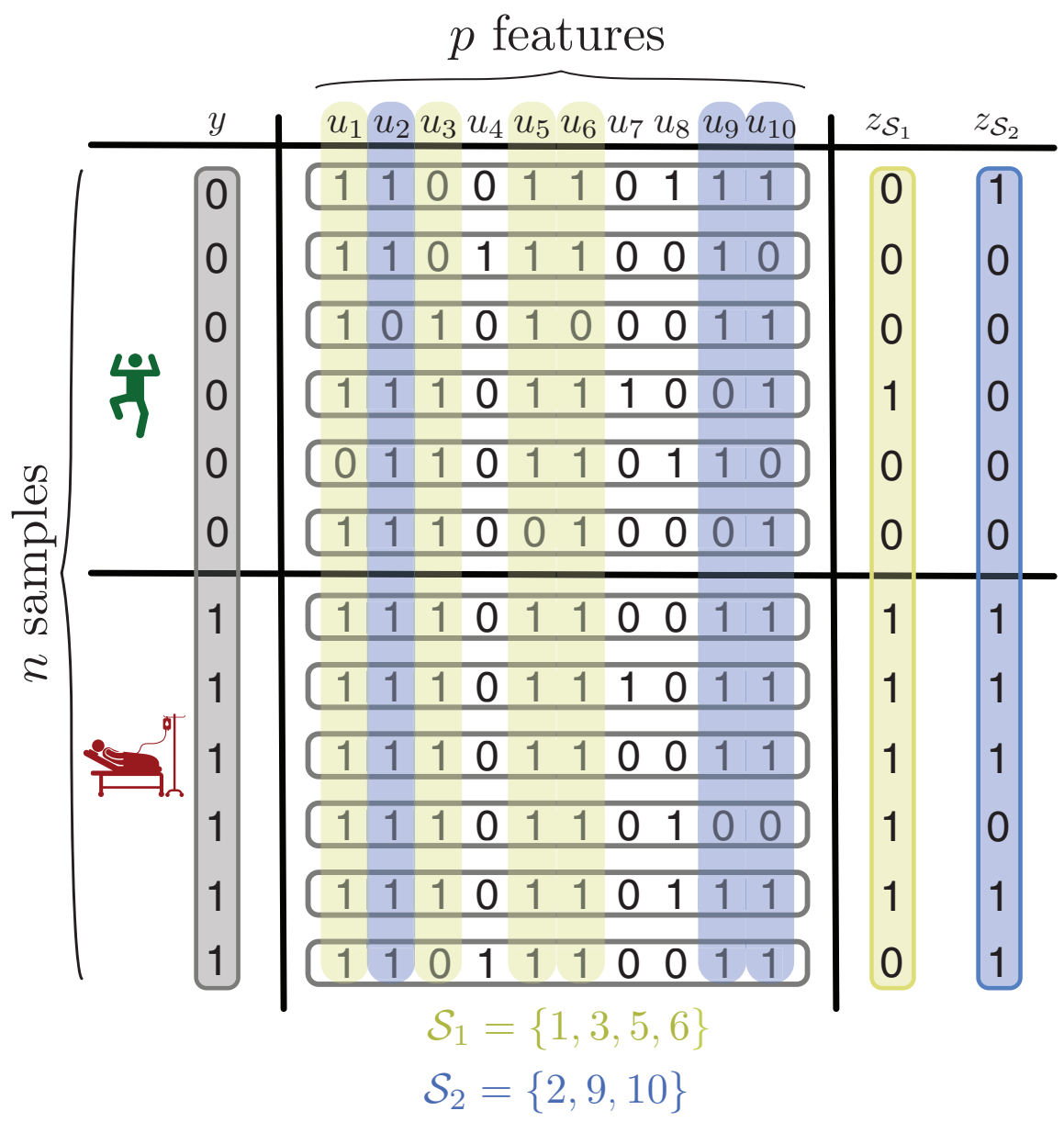

Figure 8.1: Illustration of significant itemset mining. A toy dataset with $n=12$ samples, divided into $n_{1}=6$ cases and $n_{0}=6$ controls, is shown. Each sample is represented by $p=10$ binary features, $u_{1}, u_{2}, \ldots, u_{10}$. The high-order interactions $z_{\mathcal{S}_{1}}(x)$ and $z_{\mathcal{S}_{2}}(x)$, indexed by patterns $\mathcal{S}_{1}=\{1,3,5,6\}$ (yellow) and $\mathcal{S}_{2}=\{2,9,10\}$ (blue), can be seen to be active considerably more often in cases than controls. However, none of the binary features in these patterns are individually associated with the case/control status.

even in the absence of univariate associations strongly motivates the development of methods that are able to explore arbitrary feature interactions in high-dimensional datasets. Otherwise, many signals of practical importance might remain undiscovered by standard analyses.

\subsubsection{Significant Subgraph Mining}

Another instance of significant pattern mining of great relevance occurs when each input sample is a graph, i.e., $\mathcal{X}=\left\{x \mid x=\left(V, E, l_{V}, l_{E}\right)\right\}$ where $V$ is a set of vertices, $E \subseteq V \times V$ is a set of edges, $l_{V}: V \rightarrow \Sigma_{V}$ is a function that labels each vertex in the 
graph with a categorical attribute from a finite set of categories $\Sigma_{V}$ and $l_{E}: E \rightarrow \Sigma_{E}$ is a function that labels each edge in the graph with a categorical attribute from a finite set of categories $\Sigma_{E}$. In some circumstances either edges or vertices (or both) might be unlabeled, in which case $l_{E}$ and/or $l_{V}$ can be removed from the definition of $\mathcal{X}$.

Graphs are one of the most important types of structured data. For instance, they are ubiquitous in chemoinformatics, where they are often used to represent molecules by associating atoms with vertices, labeled by the atomic symbol, and edges with bonds, labeled according to the bond type [21]. Other molecular properties might be incorporated as additional labels into vertices and edges as well. Graphs can also be used to represent a wide variety of datasets such as biological pathways, co-expression networks or protein structures, among others. Graph-structured samples are also common in a clinical setting. As an example, they are often used to represent the result of scanning the brain of a patient using magnetic resonance imaging (MRI), with vertices corresponding to a set of predefined brain regions and edges modelling the connectivity between them [22, 23].

In significant subgraph mining, a pattern $\mathcal{S}$ corresponds to a specific induced subgraph of at least one input sample in the graph dataset $\mathcal{D} .^{2}$ Typically, the search space of candidate patterns $\mathcal{M}$ is defined as the set of all distinct subgraphs of input graphs in the dataset of interest $\mathcal{D}$. Analogously to the case of significant itemset mining, the number of subgraphs one can enumerate grows combinatorially with the size of the input graphs, resulting in an enormous search space $\mathcal{M}$. A pattern $\mathcal{S}$ occurs in an input sample $x \in \mathcal{X}$, i.e. $\mathcal{S} \subseteq x$, if and only if $\mathcal{S}$ is an induced subgraph of $x$. Therefore, given a labeled graph database $\mathcal{D}$, significant subgraph mining aims to find those subgraphs $\mathcal{S}$ that occur statistically significantly more often in one class of graphs than in the other.

Figure 8.2 shows a standard application of significant subgraph mining to a toy dataset with $n=8$ graphs, $n_{1}=4$ of which belong to class $y=1$ (successful drugs) and $n_{0}=4$ of which belong to class $y=0$ (drugs that generate an adverse reaction). Each sample (drug) is represented by a graph with labeled vertices $\left(\left|\Sigma_{V}\right|=3\right)$ and labeled edges $\left(\left|\Sigma_{E}\right|=2\right.$ ). Two patterns, or subgraphs, $\mathcal{S}_{1}$ (yellow) and $\mathcal{S}_{2}$ (blue), are highlighted in the figure. Their respective occurrence indicators $g_{\mathcal{S}_{1}}$ and $g_{\mathcal{S}_{2}}$ are shown on the right of the figure, with entries in the vectors corresponding to graphs in clockwise order for each of the two classes separately. In this example, subgraph $\mathcal{S}_{1}$ is strongly overrepresented in class $y=1$ while $\mathcal{S}_{2}$ is enriched in class $y=0$. Note that, as in significant itemset mining, univariate associations analyses that only inspect individual nodes and/or edges would miss those associations.

\subsubsection{Statistical Association Testing in Significant Pattern Mining}

Regardless of the specific type of input domain $\mathcal{X}$ and patterns $\mathcal{S} \in \mathcal{M}$ of interest, the general problem to be solved in significant pattern mining is to efficiently find

2 An induced subgraph $\mathcal{S}$ of a graph $x$ is another graph, formed by selecting a subset of vertices in $x$ and keeping only the edges which connect any pair of the selected vertices. 


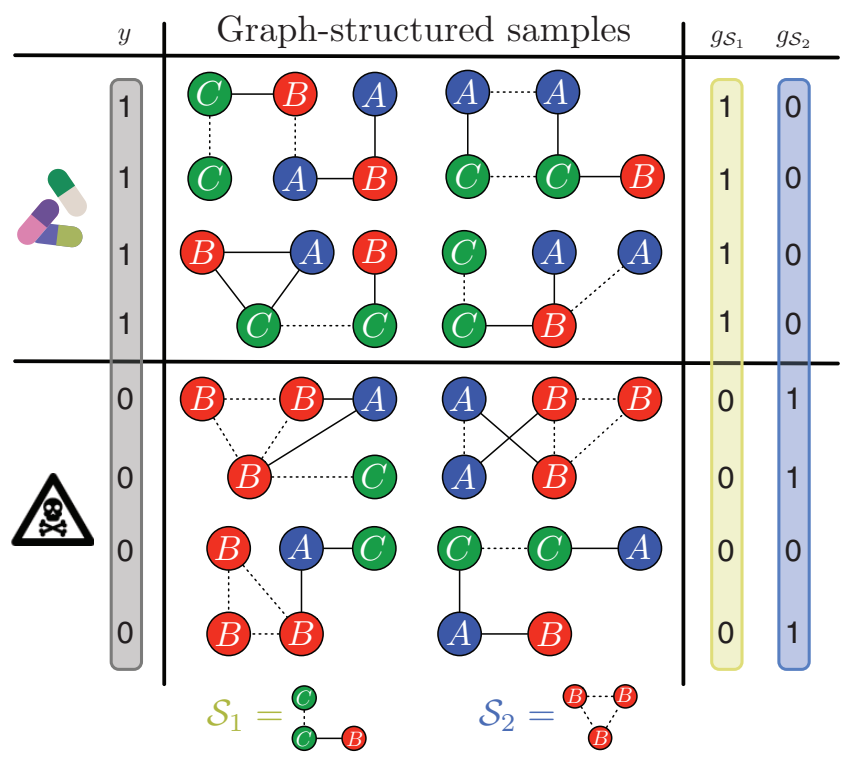

Figure 8.2: Illustration of significant subgraph mining. A toy dataset with $n=8$ samples, divided into $n_{1}=4$ successful drugs and $n_{0}=4$ drugs that trigger an adverse reaction, is shown. Each sample is represented by a graph with labeled vertices and edges. In this simple example, vertex labels belong to an alphabet with $\left|\Sigma_{V}\right|=3$ characters (blue/red/green) while edge labels belong to an alphabet with $\left|\Sigma_{E}\right|=2$ characters (solid/discontinuous). Two patterns, $\mathcal{S}_{1}$ (yellow) and $\mathcal{S}_{2}$ (blue), can be seen to occur (be induced subgraphs) significantly more often in one class of drugs than in the other.

all patterns $\mathcal{S} \in \mathcal{M}$ such that the random variable $G_{\mathcal{S}}(X)$ is statistically associated with the random variable $Y$. This naturally leads to the problem of testing the statistical association of two binary random variables, which we discuss in detail in this section.

Two random variables $G$ and $Y$ are said to be statistically associated if they are not statistically independent, i.e., if their joint probability distribution $\operatorname{Pr}(G=g, Y=y)$ does not factorize as $\operatorname{Pr}(G=g, Y=y)=\operatorname{Pr}(G=g) \operatorname{Pr}(Y=y)$. This is equivalent to the conditional probability distributions not being identical to the marginal distributions, i.e., if there exists $(g, y)$ such that $\operatorname{Pr}(G=g \mid Y=y) \neq \operatorname{Pr}(G=g)$ and there exists $(g, y)$ such that $\operatorname{Pr}(Y=y \mid G=g) \neq \operatorname{Pr}(Y=y)$. We denote the fact that random variables $G_{\mathcal{S}}(X)$ and $Y$ are statistically independent as $G_{\mathcal{S}}(X) \Perp Y$. See [24] for a general introduction to statistical independence. (See Box 8.1.)

When both random variables $G$ and $Y$ are binary, as is the case in significant pattern mining, their joint distribution $\operatorname{Pr}(G=g, Y=y)$ is fully determined by four quantities:

1. $p_{1,1}=\operatorname{Pr}(G=1, Y=1)$.

2. $p_{0,1}=\operatorname{Pr}(G=0, Y=1)$.

3. $p_{0,0}=\operatorname{Pr}(G=0, Y=0)$.

4. $p_{1,0}=\operatorname{Pr}(G=1, Y=0)$. 
Box 8.1: Joint, marginal, and conditional probabilities

Let $G$ and $Y$ be two random variables taking values in finite alphabets $\mathcal{G}$ and $\mathcal{Y}$, respectively.

- For each ordered pair $(g, y) \in \mathcal{G} \times \mathcal{Y}$, the joint probability of $g$ and $y$,

$\operatorname{Pr}(G=g, Y=y)$, denotes the probability of the joint event

$[G=g] \cap[Y=y]$. These probabilities must satisfy $\operatorname{Pr}(G=g, Y=y) \geq 0$ for all $(g, y) \in \mathcal{G} \times \mathcal{Y}$ and $\sum_{g \in \mathcal{G}} \sum_{y \in \mathcal{Y}} \operatorname{Pr}(G=g, Y=y)=1$.

- The marginal probabilities of $g$ and $y, \operatorname{Pr}(G=g)$ and $\operatorname{Pr}(Y=Y)$, denote the probabilities of the events $[G=g]$ and $[Y=y]$, respectively. Since the event $[G=g]$ can be expressed as a union of $|\mathcal{Y}|$ disjoint events, $[G=g]=\bigcup_{y \mathcal{Y}}[G=g] \cap[Y=y]$, it follows from the axioms of probability that the joint and marginal probabilities are related by $\operatorname{Pr}(G=g)=\sum_{y \in \mathcal{Y}} \operatorname{Pr}(G=g, Y=y)$, an operation commonly referred to as marginalization. Marginalization leads to valid probabilities, as it can be readily seen that $\operatorname{Pr}(G=g) \geq 0$ for all $g \in \mathcal{G}$ and that $\sum_{g \in \mathcal{G}} \operatorname{Pr}(G=g)=1$. Analogously, $\operatorname{Pr}(Y=y)=\sum_{g \in \mathcal{G}} \operatorname{Pr}(G=g, Y=y)$, where $\operatorname{Pr}(Y=y) \geq 0$ for all $y \in \mathcal{Y}$ and $\sum_{y \in \mathcal{Y}} \operatorname{Pr}(Y=y)=1$.

- Finally, the conditional probabilities of $g$ given that $[Y=y]$ and of $y$ given that $[G=g], \operatorname{Pr}(G=g \mid Y=y)$ and $\operatorname{Pr}(Y=y \mid G=g)$, denote the probability of the joint event $[G=g] \cap[Y=y]$ when the sample space has been reduced from the original set of all possible outcomes, $\bigcup_{(g, y) \in \mathcal{G} \times \mathcal{Y}}[G=g] \cap[Y=y]$, to the sets of outcomes $[Y=y]=\bigcup_{g \in \mathcal{G}}[G=g] \cap[Y=y]$ and $[G=g]=\bigcup_{g \in \mathcal{G}}[G=g] \cap[Y=y]$, respectively. Owing to the definition of conditional probability, it follows that $\operatorname{Pr}(G=g \mid Y=y)=\frac{\operatorname{Pr}(G=g, Y=y)}{\operatorname{Pr}(Y=y)}$ and $\operatorname{Pr}(Y=y \mid G=g)=\frac{\operatorname{Pr}(G=g, Y=y)}{\operatorname{Pr}(G=g)}$. Note that these probabilities are undefined if $\operatorname{Pr}(Y=y)=0$ or $\operatorname{Pr}(G=g)=0$, respectively.

This can be represented by means of a $2 \times 2$ contingency table:

\begin{tabular}{|c|c|c|c|}
\hline Variables & $G=1$ & $G=0$ & Row totals \\
\hline$Y=1$ & $p_{1,1}$ & $p_{0,1}$ & $p_{Y}$ \\
\hline$Y=0$ & $p_{1,0}$ & $p_{0,0}$ & $1-p_{Y}$ \\
\hline Col. totals & $p_{G}$ & $1-p_{G}$ & 1 \\
\hline
\end{tabular}

Since $\operatorname{Pr}(G=g, Y=y)$ must be a valid probability distribution, $p_{1,1}+p_{0,1}+p_{0,0}+p_{1,0}=$ 1 , thus leaving three degrees of freedom. The marginal distributions of $G$ and $Y$ follow from the joint distribution: $\operatorname{Pr}(G=1)=p_{1,1}+p_{1,0}=p_{G}$ and $\operatorname{Pr}(Y=1)=p_{1,1}+p_{0,1}=p_{Y}$. 
The binary random variables $G$ and $Y$ will be statistically independent and, hence, not statistically associated, if the joint distribution factorizes as $\operatorname{Pr}(G=g, Y=y)=$ $\operatorname{Pr}(G=g) \operatorname{Pr}(Y=y)$ for all $(g, y) \in\{0,1\}^{2}$. It can be shown that if the distribution factorizes for any of the four possible values of $(g, y) \in\{0,1\}^{2}$, it will also factorize for the other three. ${ }^{3}$ Therefore, without loss of generality, two binary random variables $G$ and $Y$ are statistically independent if and only if $\operatorname{Pr}(G=1, Y=1)=\operatorname{Pr}(G=1) \operatorname{Pr}(Y=$ $1)$, that is, if and only if $p_{1,1}=p_{Y} p_{G}$. Equivalently, the independence condition can also be expressed in terms of conditional distributions. Two binary random variables $G$ and $Y$ are statistically independent if and only if $\operatorname{Pr}(G=1 \mid Y=1)=\operatorname{Pr}(G=1)$. Again, the condition needs to be verified only for one of the four possible values of $(g, y) \in\{0,1\}^{2}$ as all other cases follow automatically. ${ }^{4}$

In practice, however, the joint distribution between the two binary random variables whose association is to be tested is unknown. Therefore, none of those independence conditions can be readily checked. In the context of significant pattern mining, when testing the association between the occurrence of a pattern $G_{\mathcal{S}}(X)$ and the class labels $Y$, all we have access to are $n$ i.i.d. samples from the joint distribution $\left\{\left(g_{\mathcal{S}}\left(x_{i}\right), y_{i}\right)\right\}_{i=1}^{n}$ obtained from a dataset $\mathcal{D}=\left\{\left(x_{i}, y_{i}\right)\right\}_{i=1}^{n}$. Using those $n$ samples, the unknown joint distribution $\operatorname{Pr}\left(G_{\mathcal{S}}(X)=g_{\mathcal{S}}(x), Y=y\right)$ can be approximated by counting the number of times the events $\left(g_{\mathcal{S}}(x)=1, y=1\right),\left(g_{\mathcal{S}}(x)=0, y=1\right)$, $\left(g_{\mathcal{S}}(x)=0, y=0\right)$, and $\left(g_{\mathcal{S}}(x)=1, y=0\right)$ are observed. This procedure is also typically represented as a $2 \times 2$ contingency table:

\begin{tabular}{|c|c|c|c|}
\hline Variables & $g_{\mathcal{S}}(x)=1$ & $g_{\mathcal{S}}(x)=0$ & Row totals \\
\hline$y=1$ & $a_{\mathcal{S}}$ & $b_{\mathcal{S}}$ & $n_{1}$ \\
\hline$y=0$ & $d_{\mathcal{S}}$ & $c_{\mathcal{S}}$ & $n_{0}$ \\
\hline Col. totals & $r_{\mathcal{S}}$ & $q_{\mathcal{S}}$ & $n$ \\
\hline
\end{tabular}

where,

1. $a_{\mathcal{S}}=\sum_{i=1}^{n} g_{\mathcal{S}}\left(x_{i}\right) y_{i}$ is the number of times the event $\left(g_{\mathcal{S}}=1, y=1\right)$ occurs.

2. $b_{\mathcal{S}}=\sum_{i=1}^{n}\left(1-g_{\mathcal{S}}\left(x_{i}\right)\right) y_{i}$ is the number of times the event $\left(g_{\mathcal{S}}=0, y=1\right)$ occurs.

3. $c_{\mathcal{S}}=\sum_{i=1}^{n}\left(1-g_{\mathcal{S}}\left(x_{i}\right)\right)\left(1-y_{i}\right)$ is the number of times the event $\left(g_{\mathcal{S}}=0, y=0\right)$ occurs.

4. $d_{\mathcal{S}}=\sum_{i=1}^{n} g_{\mathcal{S}}\left(x_{i}\right)\left(1-y_{i}\right)$ is the number of times the event $\left(g_{\mathcal{S}}=1, y=0\right)$ occurs.

These counts can be used to construct a frequentist approximation to the unknown joint probability distribution $\operatorname{Pr}\left(G_{\mathcal{S}}(X)=g_{\mathcal{S}}(x), Y=y\right): \hat{p}_{1,1}=a_{\mathcal{S}} / n, \hat{p}_{0,1}=b_{\mathcal{S}} / n$, $\hat{p}_{0,0}=c_{\mathcal{S}} / n$ and $\hat{p}_{1,0}=d_{\mathcal{S}} / n$. Again, all four quantities must add up to the total number of observations, $a_{\mathcal{S}}+b_{\mathcal{S}}+c_{\mathcal{S}}+d_{\mathcal{S}}=n$. Thus, the empirical approximation to the joint distribution also has three degrees of freedom. The empirical approximations of the marginal distributions of $G_{\mathcal{S}}(X)$ and $Y$ both follow from the approximation of the joint distribution: $\hat{p}_{G}=\left(a_{\mathcal{S}}+d_{\mathcal{S}}\right) / n=r_{\mathcal{S}} / n$ and $\hat{p}_{Y}=\left(a_{\mathcal{S}}+b_{\mathcal{S}}\right) / n=n_{1} / n$.

3 See Exercise 8.2.

4 See Exercise 8.3. 
Since the empirical approximation to the joint distribution will always incur some error due to random sampling, it is necessary to derive a procedure that accounts for the uncertainty introduced by the approximation. Roughly, statistical association testing involves the following sequence of steps:

1. Define a test statistic $T:\left\{\left(g_{\mathcal{S}}\left(x_{i}\right), y_{i}\right)\right\}_{i=1}^{n} \rightarrow \mathbb{R}$, i.e., a function that maps the $n$ i.i.d. samples to a scalar value. While any such function is a valid test statistic, not all choices will be equally useful. Qualitatively, a good test statistic must produce output values for data samples $\left\{\left(g_{\mathcal{S}}\left(x_{i}\right), y_{i}\right)\right\}_{i=1}^{n}$ obtained from statistically independent $\left(G_{\mathcal{S}}(X), Y\right)$ which are sufficiently different from the output values it produces for data samples $\left\{\left(g_{\mathcal{S}}\left(x_{i}\right), y_{i}\right)\right\}_{i=1}^{n}$ obtained from statistically associated $\left(G_{\mathcal{S}}(X), Y\right)$. In short, a useful test statistic can be thought as a scalar measure of association based on the $n$ observed samples.

2. Derive the null distribution of the test statistic $T, \operatorname{Pr}\left(T=t \mid H_{0}\right)$. This is the probability distribution of the test statistic $T$ under the null hypothesis $H_{0}=G_{\mathcal{S}}(X) \Perp Y$, i.e., under the assumption that the sample $\left\{\left(g_{\mathcal{S}}\left(x_{i}\right), y_{i}\right)\right\}_{i=1}^{n}$ is obtained from statistically independent $G_{\mathcal{S}}(X)$ and $Y$.

3. Evaluate the test statistic $T$ on the real data sample $\left\{\left(g_{\mathcal{S}}\left(x_{i}\right), y_{i}\right)\right\}_{i=1}^{n}$, obtaining a scalar value $t$. In order to take the uncertainty due to random sampling into account, the value of $t$ is related to the null distribution $\operatorname{Pr}\left(T=t \mid H_{0}\right)$ by computing a $P$-value. The $P$-value is defined as the probability that the test statistic takes a value at least as extreme as $t$, i.e., a value representing an association at least as strong, under the null hypothesis of independence. Assuming that larger values of $t$ correspond to stronger associations, the $P$-value is then computed as $p=\operatorname{Pr}\left(T \geq t \mid H_{0}\right)$.

4. If the $P$-value falls below a predefined significance threshold $\alpha$, i.e., $p \leq \alpha$, the random variables $G_{\mathcal{S}}(X)$ and $Y$ are deemed significantly associated. When testing a single pair of random variables for association, the significance threshold $\alpha$ has a clear interpretation as the Type I error of the procedure: it is the probability that random variables $G_{\mathcal{S}}(X)$ and $Y$ are deemed to be significantly associated given that they are actually statistically independent. Therefore, adjusting $\alpha$ allows the practitioner to trade off Type I error and statistical power, that is, to trade off the probability to report an association given that no association exists with the probability to discover an association given that it exists.

One must proceed with care when reporting an association according to the approach described above. From a statistical point of view, a low $P$-value only means that the null distribution $\operatorname{Pr}\left(T=t \mid H_{0}\right)$ is a poor fit to the observed value $t$. While this can occur due to $G_{\mathcal{S}}(X)$ and $Y$ being in fact statistically associated and, hence, the null hypothesis $H_{0}$ being false, there are other reasons why the model fit might be poor. For example, often the null distribution $\operatorname{Pr}\left(T=t \mid H_{0}\right)$ cannot be derived exactly and one must resort to approximations. Low $P$-values can therefore arise also as a consequence of the approximation to the null distribution being insufficiently precise. Besides, the statistical model chosen for the null distribution might be a poor fit to the observed value $t$ even when $H_{0}$ is true and $\operatorname{Pr}\left(T=t \mid H_{0}\right)$ can be evaluated exactly. This can occur due to mismatches between model assumptions and the ground-truth 
such as assuming the data to be Gaussian-distributed when the real distribution exhibits heavy tails or if the $n$ samples are not i.i.d. draws. In summary, statistically significant associations should not be treated as unquestionable discoveries but rather as potential associations that are worth investigating further.

In the remainder of this section, we will introduce two of the most commonly used test statistics for statistical association testing between two binary random variables: Pearson's $\chi^{2}$ test [25] and Fisher's exact test [26]. While a mathematically rigorous derivation of either test is outside the scope of this chapter, we will provide informal derivations, with emphasis on building intuition about both test statistics. For a formal derivation of the tests, we refer the reader to the original articles [25, 26].

Both Pearson's $\chi^{2}$ test and Fisher's exact test are based on $2 \times 2$ contingency tables. Without loss of generality, the data sample $\left\{\left(g_{\mathcal{S}}\left(x_{i}\right), y_{i}\right)\right\}_{i=1}^{n}$ can be summarized by the counts $a_{\mathcal{S}}, r_{\mathcal{S}}$, and $n_{1}$ which, together with the sample size $n$, uniquely determine all other counts in the contingency table. Both Pearson's $\chi^{2}$ test and Fisher's exact test make the assumption that the counts $r_{\mathcal{S}}$ and $n_{1}$ contain little information about the potential existence of an association between the two binary random variables. This is motivated by the observation that $r_{\mathcal{S}}$ is related to the marginal distribution of $G_{\mathcal{S}}(X)$ while $n_{1}$ is related to the marginal distribution of $Y$. As a consequence, both test statistics consider $r_{\mathcal{S}}$ and $n_{1}$ as fixed quantities, leaving the count $a_{\mathcal{S}}$ as the only random quantity in the model. Thus, the null distribution for both tests will be of the form $\operatorname{Pr}\left(T\left(A_{\mathcal{S}}\right)=t \mid R_{\mathcal{S}}=r_{\mathcal{S}}, N_{1}=n_{1}, H_{0}\right)$, with the specific choice of function $T\left(A_{\mathcal{S}}\right)$ being the only aspect differentiating both tests.

In order to derive the null distributions of Pearson's $\chi^{2}$ test and Fisher's exact test, we will first derive the conditional probability distribution $\operatorname{Pr}\left(A_{\mathcal{S}}=a_{\mathcal{S}} \mid R_{\mathcal{S}}=\right.$ $\left.r_{\mathcal{S}}, N_{1}=n_{1}, H_{0}\right)$ under the null hypothesis of independence $H_{0}=G_{\mathcal{S}}(X) \Perp Y$. We summarize the result in the following proposition.

Proposition 8.1 The conditional probability distribution of $A_{\mathcal{S}}$ given that $R_{\mathcal{S}}=r_{\mathcal{S}}$, $N_{1}=n_{1}$ and sample size $n$ under the null hypothesis of independence $H_{0}=G_{\mathcal{S}}(X) \Perp Y$ is a hypergeometric distribution with parameters $n, n_{1}$ and $r_{\mathcal{S}}$ :

$$
\begin{aligned}
\operatorname{Pr}\left(A_{\mathcal{S}}=a_{\mathcal{S}} \mid R_{\mathcal{S}}=r_{\mathcal{S}}, N_{1}=n_{1}, H_{0}\right) & =\operatorname{Hypergeom}\left(a_{\mathcal{S}} \mid n, n_{1}, r_{\mathcal{S}}\right) \\
& =\frac{\left(\begin{array}{c}
n_{1} \\
a_{\mathcal{S}}
\end{array}\right)\left(\begin{array}{c}
n-n_{1} \\
r_{\mathcal{S}}-a_{\mathcal{S}}
\end{array}\right)}{\left(\begin{array}{c}
n \\
r_{\mathcal{S}}
\end{array}\right)} .
\end{aligned}
$$

Proof By definition of conditional probability distribution we have:

$$
\operatorname{Pr}\left(A_{\mathcal{S}}=a_{\mathcal{S}} \mid R_{\mathcal{S}}=r_{\mathcal{S}}, N_{1}=n_{1}, H_{0}\right)=\frac{\operatorname{Pr}\left(A_{\mathcal{S}}=a_{\mathcal{S}}, R_{\mathcal{S}}=r_{\mathcal{S}} \mid N_{1}=n_{1}, H_{0}\right)}{\operatorname{Pr}\left(R_{\mathcal{S}}=r_{\mathcal{S}} \mid N_{1}=n_{1}, H_{0}\right)} .
$$

Since $R_{\mathcal{S}}=A_{\mathcal{S}}+D_{\mathcal{S}}$, the joint distribution in the numerator can be rewritten as $\operatorname{Pr}\left(A_{\mathcal{S}}=a_{\mathcal{S}}, R_{\mathcal{S}}=r_{\mathcal{S}} \mid N_{1}=n_{1}, H_{0}\right)=\operatorname{Pr}\left(A_{\mathcal{S}}=a_{\mathcal{S}}, D_{\mathcal{S}}=r_{\mathcal{S}}-a_{\mathcal{S}} \mid N_{1}=n_{1}, H_{0}\right)$. Note that $A_{\mathcal{S}}$ depends only on samples for which $y_{i}=1$ while $D_{\mathcal{S}}$ depends only on samples for which $y_{i}=0$. Therefore, under the assumption that all $n$ samples are i.i.d. draws, the random variables $A_{\mathcal{S}}$ and $D_{\mathcal{S}}$ are statistically independent. This allows the joint distribution in the numerator to be decomposed as $\operatorname{Pr}\left(A_{\mathcal{S}}=a_{\mathcal{S}}, R_{\mathcal{S}}=r_{\mathcal{S}} \mid N_{1}=\right.$ $\left.n_{1}, H_{0}\right)=\operatorname{Pr}\left(A_{\mathcal{S}}=a_{\mathcal{S}} \mid N_{1}=n_{1}, H_{0}\right) \operatorname{Pr}\left(D_{\mathcal{S}}=r_{\mathcal{S}}-a_{\mathcal{S}} \mid N_{1}=n_{1}, H_{0}\right)$. 
Let $p_{1 \mid 1}=\operatorname{Pr}\left(G_{\mathcal{S}}=1 \mid Y=1\right)$ and $p_{1 \mid 0}=\operatorname{Pr}\left(G_{\mathcal{S}}=1 \mid Y=0\right)$. If the null hypothesis of independence $H_{0}$ holds, then $p_{1 \mid 1}=p_{1 \mid 0}=p_{G}$. If the $n$ samples are obtained as i.i.d. draws, $A_{\mathcal{S}}$ can be modeled as the sum of $n_{1}$ independent Bernoulli random variables, ${ }^{5}$ each with success probability $p_{G}$. Hence, ${ }^{6} \operatorname{Pr}\left(A_{\mathcal{S}}=a_{\mathcal{S}} \mid N_{1}=n_{1}, H_{0}\right)=\operatorname{Binomial}\left(a_{\mathcal{S}} \mid\right.$ $\left.n_{1}, p_{G}\right)=\left(\begin{array}{l}n_{1} \\ a_{\mathcal{S}}\end{array}\right) p_{G}^{a_{\mathcal{S}}}\left(1-p_{G}\right)^{n-a_{\mathcal{S}}}$. Analogously, $D_{\mathcal{S}}$ can be modeled as the sum of $n-$ $n_{1}$ independent Bernoulli random variables, each with success probability $p_{G}$. Hence, $\operatorname{Pr}\left(D_{\mathcal{S}}=r_{\mathcal{S}}-a_{\mathcal{S}} \mid N_{1}=n_{1}, H_{0}\right)=\operatorname{Binomial}\left(r_{\mathcal{S}}-a_{\mathcal{S}} \mid n-n_{1}, p_{G}\right)=\left(\begin{array}{c}n-n_{1} \\ r_{\mathcal{S}}-a_{\mathcal{S}}\end{array}\right) p_{G}^{r_{\mathcal{S}}-a_{\mathcal{S}}}(1-$ $\left.p_{G}\right)^{\left(n-n_{1}\right)-\left(r_{\mathcal{S}}-a_{\mathcal{S}}\right)}$. Finally, since $R_{\mathcal{S}}=A_{\mathcal{S}}+D_{\mathcal{S}}$, with $A_{\mathcal{S}} \Perp D_{\mathcal{S}}, R_{\mathcal{S}}$ corresponds to a sum of $n$ Bernoulli variables with success probability $p_{G}$, leading to $\operatorname{Pr}\left(R_{\mathcal{S}}=r_{\mathcal{S}} \mid N_{1}=\right.$ $\left.n_{1}, H_{0}\right)=\operatorname{Binomial}\left(r_{\mathcal{S}} \mid n, p_{G}\right)=\left(\begin{array}{c}n \\ r_{\mathcal{S}}\end{array}\right) p_{G}^{r_{\mathcal{S}}}\left(1-p_{G}\right)^{n-r_{\mathcal{S}}}$.

Substituting those distributions into Equation (8.3) leads to the final result. In particular, the conditioning on $R_{\mathcal{S}}=r_{\mathcal{S}}$ eliminates the influence of the nuisance parameter $p_{G}$, leading to a distributional form that depends only on $n_{1}, r_{\mathcal{S}}$, and the sample size $n$.

Building on Proposition 8.1, Pearson's $\chi^{2}$ test and Fisher's exact test can be motivated as shown in the following subsections.

\subsubsection{Pearson's $\chi^{2}$ Test}

Pearson's $\chi^{2}$ test can be understood as a Z-score squared:

$$
T_{\text {Pearson }}\left(a_{\mathcal{S}} \mid n, n_{1}, r_{\mathcal{S}}\right)=\left(\frac{a_{\mathcal{S}}-\mathbb{E}\left[a_{\mathcal{S}} \mid R_{\mathcal{S}}=r_{\mathcal{S}}, N_{1}=n_{1}, H_{0}\right]}{\operatorname{Std}\left[a_{\mathcal{S}} \mid R_{\mathcal{S}}=r_{\mathcal{S}}, N_{1}=n_{1}, H_{0}\right]}\right)^{2},
$$

where $\mathbb{E}\left[a_{\mathcal{S}} \mid R_{\mathcal{S}}=r_{\mathcal{S}}, N_{1}=n_{1}, H_{0}\right]=r_{\mathcal{S}} \frac{n_{1}}{n}$ and $\operatorname{Std}\left[a_{\mathcal{S}} \mid R_{\mathcal{S}}=r_{\mathcal{S}}, N_{1}=n_{1}, H_{0}\right]=$ $\sqrt{\frac{r_{S}}{n} \frac{n-r_{\mathcal{S}}}{n} \frac{n-n_{1}}{n-1} n_{1}}$ are the mean and standard deviation of a hypergeometric distribution with parameters $n, n_{1}$, and $r_{\mathcal{S}}$. This leads to the following expression for the test statistic:

$$
T_{\text {Pearson }}\left(a_{\mathcal{S}} \mid n, n_{1}, r_{\mathcal{S}}\right)=\frac{\left(a_{\mathcal{S}}-r_{\mathcal{S}} \frac{n_{1}}{n}\right)^{2}}{\frac{r_{\mathcal{S}}}{n} \frac{n-r_{\mathcal{S}}}{n} \frac{n-n_{1}}{n-1} n_{1}}
$$

Intuitively, large values of $T_{\text {Pearson }}\left(a_{\mathcal{S}} \mid n, n_{1}, r_{\mathcal{S}}\right)$ are less likely to occur under the null hypothesis, hence being suggestive of the potential existence of an association.

If the sample size $n$ is sufficiently large, the central limit theorem implies that the underlying Z-score defining Pearson's $\chi^{2}$ test will be approximately distributed as a standard Gaussian under the null hypothesis $H_{0} .{ }^{7}$ Therefore, for large $n$, the null

5 The Bernoulli distribution represents the probability distribution of a random variable $Y$ which can only attain two possible outcomes: $Y=1$, often generically denoted as "success" and $Y=0$, often referred to as "failure." Typical examples of phenomena which can be modelled with a Bernoulli distribution include a coin toss or the answer to a yes/no question. Given the success probability $p$, the Bernoulli distribution can be written as $\operatorname{Pr}(Y=y \mid p)=\operatorname{Bernoulli}(y \mid p)=p^{y}(1-p)^{1-y}$.

6 The binomial distribution arises as the probability distribution of an integer-valued random variable $Z$ describing the total number of "successes" out of $n$ independent Bernoulli trials, all having the same success probability $p$. The relationship between the Bernoulli distribution and the binomial distribution is explored in-depth in Exercise 8.7.

7 Informally, the central limit theorem asserts that the average of $n$ i.i.d. random variables with zero mean and unit variance is approximately distributed as a standard Gaussian provided that $n$ is large enough. 
distribution of the test statistic $\operatorname{Pr}\left(T_{\text {Pearson }}\left(A_{\mathcal{S}}\right)=t \mid R_{\mathcal{S}}=r_{\mathcal{S}}, N_{1}=n_{1}, H_{0}\right)$ can be approximated as a $\chi_{1}^{2}$ distribution. The corresponding two-tailed $P$-value can then be computed from the survival function of a $\chi_{1}^{2}$ distribution, i.e.,

$$
p_{\text {Pearson }}\left(a_{\mathcal{S}} \mid n, n_{1}, r_{\mathcal{S}}\right)=1-F_{\chi_{1}^{2}}\left(T_{\text {Pearson }}\left(a_{\mathcal{S}} \mid n, n_{1}, r_{\mathcal{S}}\right)\right)
$$

where $F_{\chi_{1}^{2}}(\bullet)$ is the cumulative density function of a $\chi_{1}^{2}$ distribution.

\subsubsection{Fisher's Exact Test}

While there are multiple ways to compute two-tailed $P$-values for Fisher's exact test, in this chapter we focus on one particular form for the sake of clarity. However, all subsequent derivations can be adapted to work with other definitions of the two-tailed $P$-value, as well as for one-tailed $P$-values.

A concise way to motivate Fisher's exact test is to consider the probability $\operatorname{Pr}\left(A_{\mathcal{S}}=\right.$ $\left.a_{\mathcal{S}} \mid R_{\mathcal{S}}=r_{\mathcal{S}}, N_{1}=n_{1}, H_{0}\right)$ as the test statistic itself:

$$
T_{\text {Fisher }}\left(a_{\mathcal{S}} \mid n, n_{1}, r_{\mathcal{S}}\right)=\operatorname{Hypergeom}\left(a_{\mathcal{S}} \mid n, n_{1}, r_{\mathcal{S}}\right)=\frac{\left(\begin{array}{c}
n_{1} \\
a_{\mathcal{S}}
\end{array}\right)\left(\begin{array}{c}
n-n_{1} \\
r_{\mathcal{S}}-a_{\mathcal{S}}
\end{array}\right)}{\left(\begin{array}{c}
n \\
r_{\mathcal{S}}
\end{array}\right)} .
$$

This definition is intuitive, as $T_{\text {Fisher }}\left(a_{\mathcal{S}} \mid n, n_{1}, r_{\mathcal{S}}\right)$ will be small for values of $a_{\mathcal{S}}$ which are improbable under the conditional null distribution $\operatorname{Pr}\left(A_{\mathcal{S}}=a_{\mathcal{S}} \mid R_{\mathcal{S}}=\right.$ $\left.r_{\mathcal{S}}, N_{1}=n_{1}, H_{0}\right)$. Thus, in this case, low values of the test statistic $T_{\text {Fisher }}\left(a_{\mathcal{S}} \mid n, n_{1}, r_{\mathcal{S}}\right)$, rather than large values, are indicative of a potential association. As a consequence, to obtain a two-tailed $P$-value, we will compute $p=\operatorname{Pr}\left(T \leq t \mid H_{0}\right)$ rather than $p=\operatorname{Pr}\left(T \geq t \mid H_{0}\right)$.

Let $\mathcal{A}\left(a_{\mathcal{S}}\right)=\left\{a_{\mathcal{S}}^{\prime} \mid\right.$ Hypergeom $\left.\left(a_{\mathcal{S}}^{\prime} \mid n, n_{1}, r_{\mathcal{S}}\right) \leq \operatorname{Hypergeom}\left(a_{\mathcal{S}} \mid n, n_{1}, r_{\mathcal{S}}\right)\right\}$ be the set of all possible counts $a_{\mathcal{S}}^{\prime}$ that are at least as improbable as $a_{\mathcal{S}}$ under the conditional null distribution. Then:

$$
p_{\text {Fisher }}\left(a_{\mathcal{S}} \mid n, n_{1}, r_{\mathcal{S}}\right)=\sum_{a_{\mathcal{S}}^{\prime} \in \mathcal{A}\left(a_{\mathcal{S}}\right)} \operatorname{Hypergeom}\left(a_{\mathcal{S}}^{\prime} \mid n, n_{1}, r_{\mathcal{S}}\right)
$$

Unlike Pearson's $\chi^{2}$ test and as its name indicates, Fisher's exact test does not need to resort to approximations to model the null distribution. Therefore, it is often the preferred choice, specially in situations where the sample size $n$ is small.

\subsubsection{Multiple Testing Correction}

In the previous section, we introduced Pearson's $\chi^{2}$ test and Fisher's exact test, two methods to test the statistical association of two binary random variables based on $n$ i.i.d. samples. In the context of significant pattern mining, these can be used to test if the occurrence of a pattern $\mathcal{S} \in \mathcal{M}$ in an input sample $x \in \mathcal{X}$ is statistically associated with the sample class $y$ based on a dataset $\mathcal{D}=\left\{\left(x_{i}, y_{i}\right)\right\}_{i=1}^{n}$. In principle, a $P$-value $p_{\mathcal{S}}$ could be computed for each pattern $\mathcal{S} \in \mathcal{M}$, deeming those patterns $\mathcal{S} \in \mathcal{M}$ for which $p_{\mathcal{S}} \leq \alpha$ statistically significant. However, leaving the matter of 
computational feasibility temporarily aside, such an approach also has an important statistical caveat. A procedure that, independently of the number $|\mathcal{M}|$ of association tests being performed, uses a fixed significance threshold $\alpha$ to assess the significance of each pattern $\mathcal{S} \in \mathcal{M}$, will generate on average $\alpha\left|\mathcal{M}_{\text {null }}\right|$ false positives, where $\mathcal{M}_{\text {null }}$ is the set of patterns that are statistically independent of the class labels. In most applications of interest, there is an enormous number $|\mathcal{M}|$ of candidate patterns to be tested, often in the order of trillions, most of which belong to the set of null patterns $\mathcal{M}_{\text {null }}$. Therefore, if the statistical association testing procedure is not modified to account for the vast number of association tests being performed simultaneously, billions of false positives will be reported on average, rendering the results entirely unreliable. This phenomenon, commonly referred to as the multiple hypothesis testing problem, has been thoroughly studied by statisticians (e.g., [27, 28, 29]). However, significant pattern mining takes the multiple hypothesis testing problem to a completely new level, dealing with a number $|\mathcal{M}|$ of association tests many orders of magnitude larger than it had ever been considered before in other domains.

A key concept when dealing with the multiple hypothesis testing problem is the family-wise error rate (FWER), defined as the probability of producing any false positives in the analysis. Mathematically, $\operatorname{FWER}(\delta)=\operatorname{Pr}(\operatorname{FP}(\delta)>0)$, where $\operatorname{FP}(\delta)$ is the number of false positives at adjusted significance threshold $\delta$, i.e., the number of patterns $\mathcal{S} \in \mathcal{M}_{\text {null }}$ for which $p_{\mathcal{S}} \leq \delta$. A common way to account for the multiple hypothesis testing problem is to modify the statistical association testing procedure in order to control the FWER at level $\alpha$, i.e., to guarantee that the FWER is bounded above by a user-defined level $\alpha$. Since, by definition, $\operatorname{FWER}(\delta)$ is monotonically increasing in the adjusted significance threshold $\delta$, the optimal significance threshold that controls the FWER at level $\alpha$ is given by:

$$
\delta^{*}=\max \{\delta \mid \operatorname{FWER}(\delta) \leq \alpha\}
$$

Any values of the adjusted significance threshold $\delta$ that are larger than $\delta^{*}$ will fail the control the FWER at level $\alpha$, while values that are smaller than $\delta^{*}$ will also control the FWER at level $\alpha$ but yield less statistical power to discover the true associations.

While FWER control offers an elegant framework to account for the multiple hypothesis testing problem, a fundamental limitation remains: $\operatorname{FWER}(\delta)$ is an unknown quantity that depends on the (unknown) joint distribution of $\left\{p_{\mathcal{S}} \mid \mathcal{S} \in\right.$ $\left.\mathcal{M}_{\text {null }}\right\}$. Therefore, neither FWER $(\delta)$ nor $\delta^{*}$ can be computed exactly. Existing approaches resort to approximating $\operatorname{FWER}(\delta)$, often with a quantity that is provably an upper bound, and use that approximation to propose an adjusted significance threshold $\hat{\delta}^{*}$. Distinct procedures differ on the quality of their approximation of the FWER, which ultimately determines how close the resulting $\hat{\delta}^{*}$ is to the optimal adjusted significance threshold $\delta^{*}$.

\subsubsection{The Bonferroni Correction}

The most popular procedure to control the FWER is the Bonferroni correction [30, 31]. The Bonferroni correction approximates the unknown value of $\operatorname{FWER}(\delta)$ with 
$\widehat{\operatorname{FWER}}(\delta)=\delta|\mathcal{M}|$, a quantity that can be readily shown to be an upper bound on $\operatorname{FWER}(\delta)$ :

$$
\begin{aligned}
\operatorname{FWER}(\delta)=\operatorname{Pr}\left(\bigcup_{\mathcal{S} \in \mathcal{M}_{\text {null }}}\left\{p_{\mathcal{S}} \leq \delta\right\}\right) & \leq \sum_{\mathcal{S} \in \mathcal{M}_{\text {null }}} \operatorname{Pr}\left(p_{\mathcal{S}} \leq \delta\right) \\
& \leq \delta\left|\mathcal{M}_{\text {null }}\right| \leq \delta|\mathcal{M}|
\end{aligned}
$$

where $\left\{p_{\mathcal{S}} \leq \delta\right\}$ is the event that pattern $\mathcal{S}$ is deemed significantly associated. Based on the upper bound $\widehat{\operatorname{FWER}}(\delta)=\delta|\mathcal{M}|$, the Bonferroni correction proposes an adjusted significance threshold $\delta_{\text {bonf }}=\max \{\delta|\delta| \mathcal{M} \mid \leq \alpha\}=\alpha /|\mathcal{M}|$. Since $\widehat{\operatorname{FWER}}(\delta) \geq \operatorname{FWER}(\delta)$, it follows that $\delta_{\text {bonf }} \leq \delta^{*}$ and, therefore, the Bonferroni correction controls the FWER at level $\alpha$.

In practice, the Bonferroni correction tends to be an over-conservative procedure. Its approximation $\widehat{\operatorname{FWER}}(\delta)=\delta|\mathcal{M}|$ to the FWER often greatly overestimates the real value of $\operatorname{FWER}(\delta)$, causing $\delta_{\text {bonf }}$ to be considerably smaller than $\delta^{*}$ and leading to a loss of statistical power. However, the Bonferroni correction has strong points due to its simplicity: It introduces no computational overhead and ensures FWER-control for any kind of data, without the need to satisfy any assumptions. Those aspects have made the Bonferroni correction the most widespread tool for FWER-control, making the loss of statistical power it entails a price happily paid by many practitioners.

Unfortunately, the multiple hypothesis testing problem that arises in significant pattern mining is way beyond what the Bonferroni correction can successfully handle. Since the number $|\mathcal{M}|$ of candidate patterns in a typical significant pattern mining problem is enormous, the resulting adjusted significance threshold $\delta_{\text {bonf }}$ will be too small and the resulting procedure will have virtually no statistical power. The lack of approaches to control the FWER when performing such a large number of association tests caused traditional pattern mining methods to either (1) ignore the multiple hypothesis testing problem, providing a ranking of patterns by association but no statistical guarantees $[32,33,34]$ or (2) artificially limit the size of the search space $\mathcal{M}$ by setting an arbitrary maximum pattern size, in order to be able to apply a Bonferroni correction in the much smaller resulting search space [35, 36, 37].

\subsubsection{Tarone's Improved Bonferroni Correction for Discrete Data}

It was only recently that the first algorithm able to control the FWER in significant pattern mining without imposing any limits on the search space $\mathcal{M}$ was proposed [15]. A key component of their approach is the use of Tarone's improved Bonferroni correction for discrete data [38], an alternative way of approximating the FWER that results in a drastically more accurate bound when applying the procedure to pattern mining problems.

The core idea behind Tarone's method is to exploit the nature of test statistics for discrete data. Consider a $2 \times 2$ contingency table summarizing $n$ i.i.d. samples of two binary random variables with the margins $n_{1}$ and $r_{\mathcal{S}}$ treated as fixed. In order to be consistent with the fixed margins, the cell count $a_{\mathcal{S}}$ must be smaller or equal than $a_{\mathcal{S}, \max }=\min \left(n_{1}, r_{\mathcal{S}}\right)$ and larger or equal than $a_{\mathcal{S}, \min }=\max \left(0, r_{\mathcal{S}}-\left(n-n_{1}\right)\right)$. Therefore, 
$a_{\mathcal{S}}$ can only take $a_{\mathcal{S}, \max }-a_{\mathcal{S}, \min }+1$ distinct values. In turn, this implies that there are at most $a_{\mathcal{S}, \max }-a_{\mathcal{S}, \min }+1$ distinct $P$-values that can be obtained as an outcome of applying a test statistic such as Pearson's $\chi^{2}$ test or Fisher's exact test to the $2 \times 2$ contingency table. Since there is a finite number of $P$-values that can be observed, there exists a minimum attainable P-value, $p_{\mathcal{S}, \min }=\min \left\{p_{\mathcal{S}}\left(a_{\mathcal{S}}^{\prime} \mid n, n_{1}, r_{\mathcal{S}}\right) \mid a_{\mathcal{S}}^{\prime} \in \llbracket a_{\mathcal{S}, \min ,}, a_{\mathcal{S}, \max } \rrbracket\right\}$, where $p_{\mathcal{S}}\left(a_{\mathcal{S}}^{\prime} \mid n, n_{1}, r_{\mathcal{S}}\right)$ is the $P$-value obtained by applying the test statistic of choice to a $2 \times 2$ contingency table with cell count $a_{\mathcal{S}}^{\prime}$, fixed margins $n_{1}$ and $r_{\mathcal{S}}$ and sample size $n$. The existence of a minimum attainable $P$-value $p_{\mathcal{S} \text {, min }}$ strictly larger than zero is a special property of discrete data. In principle, $P$-values obtained when testing the association between two continuous random variables can be arbitrarily close to zero. Moreover, the minimum attainable $P$-value $p_{\mathcal{S} \text {, min }}$ depends only on $n, n_{1}$ and $r_{\mathcal{S}}$. In particular, $p_{\mathcal{S} \text {, min }}$ does not depend on the actual value of the cell count $a_{\mathcal{S}}$.

The existence of a minimum attainable $P$-value $p_{\mathcal{S} \text {, min }}$ strictly larger than zero has profound implications. Suppose that the adjusted significance threshold $\delta$ is smaller than the minimum attainable $P$-value, $p_{\mathcal{S}, \min }>\delta$. Then, by definition, regardless of the value of $a_{\mathcal{S}}$, the corresponding association will not be deemed statistically significant. Consequently, it can also never cause a false positive at adjusted significance threshold $\delta$. Patterns $\mathcal{S} \in \mathcal{M}$ for which this occurs are said to be untestable at level $\delta$ while the remaining patterns are said to be testable at level $\delta$. Let $\mathcal{M}_{\text {test }}(\delta)=\left\{\mathcal{S} \in \mathcal{M} \mid p_{\mathcal{S} \text {, min }} \leq \delta\right\}$ be the set of testable patterns at level $\delta$. Tarone's improved Bonferroni correction for discrete data approximates $\operatorname{FWER}(\delta)$ as $\widehat{\operatorname{FWER}}(\delta)=\delta\left|\mathcal{M}_{\text {test }}(\delta)\right|$, which is also an upper bound on $\operatorname{FWER}(\delta)$ :

$$
\begin{aligned}
\operatorname{FWER}(\delta) & =\operatorname{Pr}\left(\bigcup_{\mathcal{S} \in \mathcal{M}_{\text {null }}}\left\{p_{\mathcal{S}} \leq \delta\right\}\right)=\operatorname{Pr}\left(\bigcup_{\mathcal{S} \in \mathcal{M}_{\text {test }}(\delta)}\left\{p_{\mathcal{S}} \leq \delta\right\}\right) \\
& \leq \sum_{\mathcal{S} \in \mathcal{M}_{\text {test }}(\delta)} \operatorname{Pr}\left(p_{\mathcal{S}} \leq \delta\right) \leq \delta\left|\mathcal{M}_{\text {test }}(\delta)\right|,
\end{aligned}
$$

where the first step follows from the fact that untestable patterns $\mathcal{S} \in \mathcal{M} \backslash \mathcal{M}_{\text {test }}(\delta)$ cannot cause a false positive at adjusted significance threshold $\delta$. Thus, Tarone's method also guarantees FWER control without the need for additional assumptions.

In significant pattern mining, often the number of testable patterns $\left|\mathcal{M}_{\text {test }}(\delta)\right|$ is drastically smaller than the total number of candidate patterns $|\mathcal{M}|$. By exploiting the discrete nature of the data, Tarone's method often results in a much tighter upper bound of $\operatorname{FWER}(\delta)$ than the original Bonferroni correction. This leads to the adjusted significance threshold $\delta_{\text {tar }}=\max \left\{\delta|\delta| \mathcal{M}_{\text {test }}(\delta) \mid \leq \alpha\right\}$ being much closer to the optimal $\delta^{*}$ than $\delta_{\text {bonf }}$, bringing forth a drastic gain of statistical power over the original Bonferroni correction and making significant pattern mining on the entire search space $\mathcal{M}$ statistically feasible.

8 Given any two integers $a$ and $b$ such that $a<b$, we denote by $\llbracket a, b \rrbracket$ the set of consecutive integers $\{a, a+1, \ldots, b\}$. 


\subsection{A Framework for Significant Pattern Mining Using Tarone's Method}

In the previous section, we have identified the multiple hypothesis testing problem as the main statistical challenge that needs to be overcome to make significant pattern mining a reality. We introduced the concept of FWER control at level $\alpha$ as a criterion for statistical association testing that corrects for the multiple hypothesis testing problem and discussed the feasibility of controlling the FWER in the setting of significant pattern mining. Standard techniques, such as the ubiquitous Bonferroni correction, are unable to maintain statistical power when exploring the massive search space of candidate patterns $\mathcal{M}$ present in typical significant pattern mining problems. However, Tarone's method leverages the concept of testability, the phenomenon that only a subset $\mathcal{M}_{\text {test }}(\delta) \subseteq \mathcal{M}$ of patterns $\left(\left|\mathcal{M}_{\text {test }}(\delta)\right| \ll|\mathcal{M}|\right)$ can be significant or cause a false positive, to dramatically enhance statistical power. However, evaluating the adjusted significance threshold $\delta_{\text {tar }}$ naively is computationally an extremely demanding task.

In order to obtain $\delta_{\text {tar, }}$, the largest value of $\delta$ that satisfies $\delta\left|\mathcal{M}_{\text {test }}(\delta)\right| \leq \alpha$ needs to be found. Obtaining $\mathcal{M}_{\text {test }}(\delta)$ by brute force would require enumerating all patterns $\mathcal{S} \in$ $\mathcal{M}$, computing all corresponding minimum attainable $P$-values $p_{\mathcal{S} \text {, min }}$ and keeping those patterns $\mathcal{S} \in \mathcal{M}$ for which $p_{\mathcal{S} \text {, min }} \leq \delta$. Due to the enormous number $|\mathcal{M}|$ of candidate patterns, this strategy is computationally unfeasible except in very small datasets. This difficulty kept Tarone's method from being used in significant pattern mining until the recent breakthrough presented in [15]. In their work, the authors propose a way to exploit properties of specific test statistics, such as Pearson's $\chi^{2}$ test and Fisher's exact test, to derive an algorithm that can efficiently compute $\delta_{\text {tar }}$ by exploring only a small subset of all candidate patterns in the search space $\mathcal{M}$. Since its publication, the original algorithm in [15] has been subsequently enhanced by followup work [16,17], improving its computational efficiency even further. In the remainder of this section, we will present a generic significant pattern mining algorithm that incorporates all these recent developments.

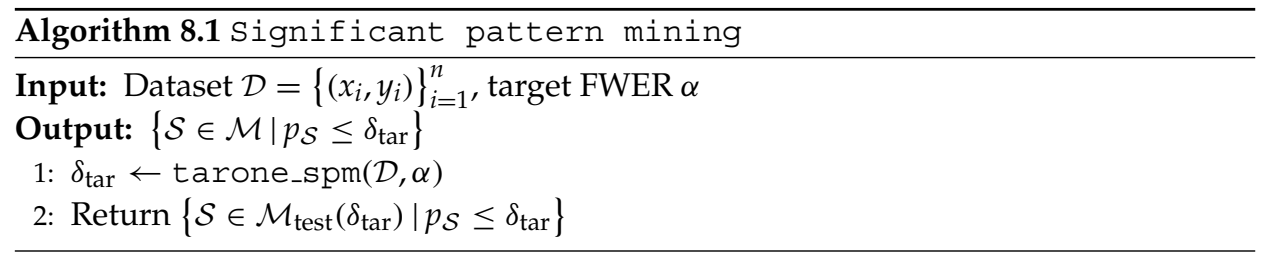

Algorithm 8.1 describes a generic significant pattern mining algorithm making use of Tarone's method. Mainly, it proceeds in two key steps:

1. Obtain Tarone's corrected significance threshold $\delta_{\mathrm{tar}}$. This step is performed in Line 1, which invokes the routine tarone_spm. This routine makes use of certain properties of the minimum attainable $P$-value to dynamically prune a large proportion of the search space $\mathcal{M}$ as patterns are enumerated.

Effectively, this allows to exactly compute $\delta_{\text {tar }}$ while only enumerating a small subset of patterns comprising the search space, as described in detail in Algorithm 8.2 below. 


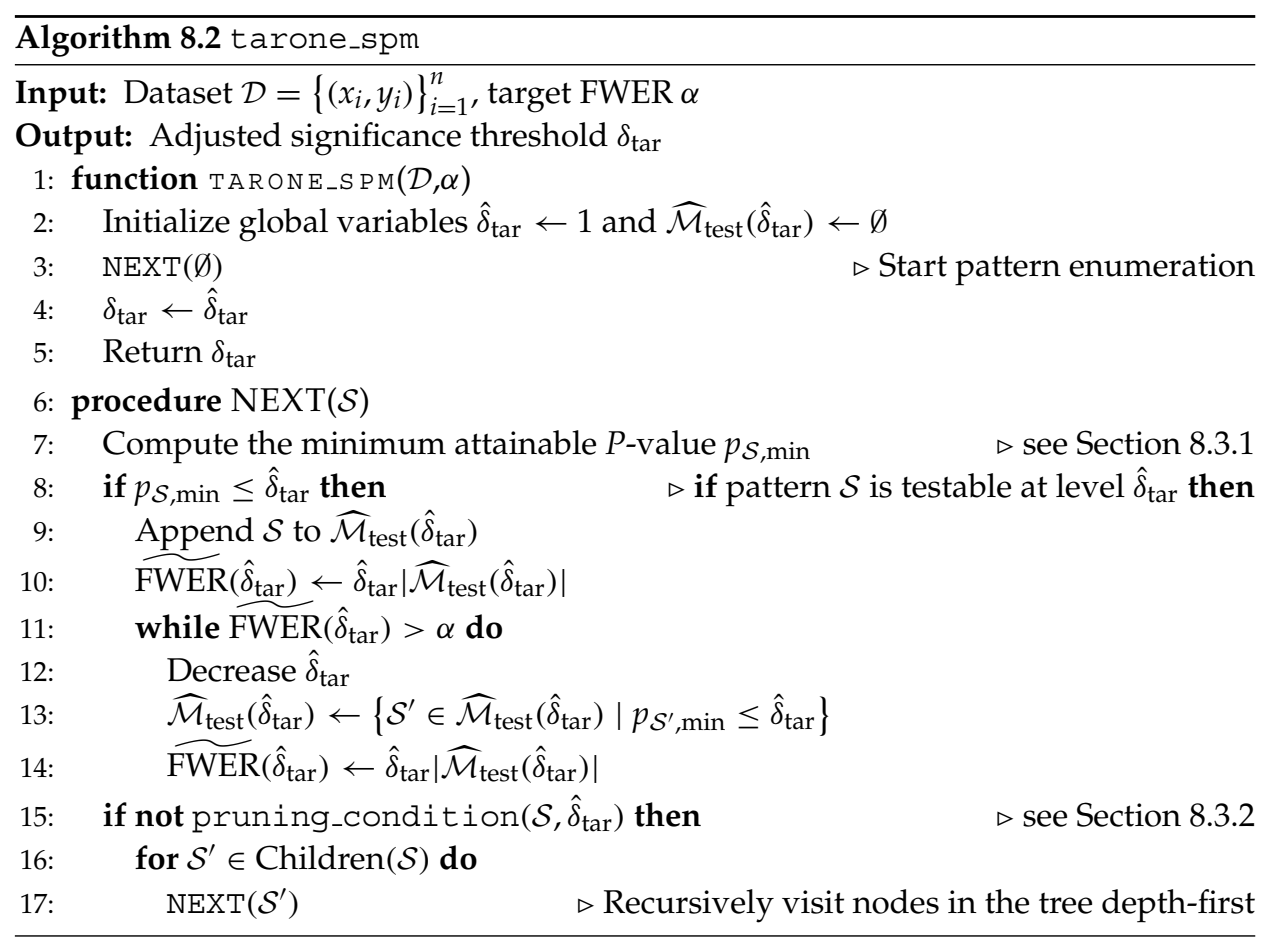

2. Compute the $P$-value $p_{\mathcal{S}}$ for all testable patterns $\mathcal{S} \in \mathcal{M}_{\text {test }}\left(\delta_{\text {tar }}\right)$, using a test statistic such as Pearson's $\chi^{2}$ test or Fisher's exact test, and output those that are statistically significant at level $\delta_{\text {tar }}$. Note that, by definition, untestable patterns $\mathcal{S} \in \mathcal{M} \backslash \mathcal{M}_{\text {test }}\left(\delta_{\text {tar }}\right)$ cannot be statistically significant at adjusted significance threshold $\delta_{\text {tar }}$ and, therefore, do not need to be taken into account in this step. Since the number of testable patterns $\left|\mathcal{M}_{\text {test }}\left(\delta_{\text {tar }}\right)\right|$ tends to be much smaller than the total number of patterns $|\mathcal{M}|$, the computational complexity of this step, performed in Line 2, is less critical than the previous step.

The routine tarone_spm is the core of the significant pattern mining algorithm described in Algorithm 8.1. The method explores the search space of candidate patterns $\mathcal{S} \in \mathcal{M}$ recursively, arranging all patterns as nodes of a tree such that the descendants $\mathcal{S}^{\prime}$ of a pattern $\mathcal{S}$ are super-patterns of $\mathcal{S}$, i.e., $\mathcal{S} \subseteq \mathcal{S}^{\prime}$. We call such a tree a pattern enumeration tree. This particular way of arranging the search space of patterns has been extensively used in pattern mining [39], and is applicable in most instances of significant pattern mining, including both significant itemset mining and significant subgraph mining. For instance, Figure 8.3 illustrates one of the many possible ways to obtain a pattern enumeration tree in a significant itemset mining problem with $p=5$ features. In this example, each node in the tree corresponds to a high-order feature interaction $\mathcal{S}$ and children $\mathcal{S}^{\prime}$ of a feature interaction $\mathcal{S}$ are obtained by adding an additional feature to the interaction. Note that, depending on the ordering of features, multiple valid trees can be obtained. Analogously, in significant subgraph mining, 


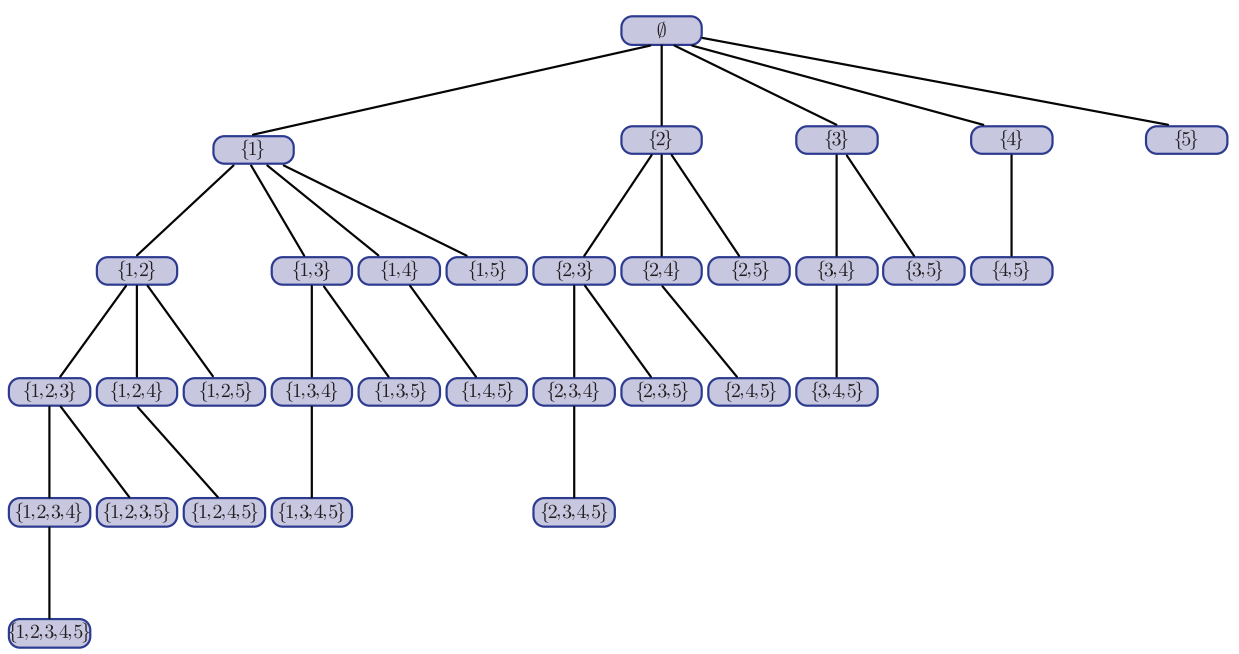

Figure 8.3: Depiction of a valid pattern enumeration tree for a significant itemset mining problem with $p=5$ features. Each of the $|\mathcal{M}|=2^{5}$ high-order interactions $\mathcal{S} \in \mathcal{M}$ is mapped to a node of the tree in such a way that $\mathcal{S}^{\prime} \in \operatorname{Children}(\mathcal{S})$ implies that $\mathcal{S} \subseteq \mathcal{S}^{\prime}$.

each node in the tree corresponds to a subgraph $\mathcal{S}$ and children $\mathcal{S}^{\prime}$ of $\mathcal{S}$ can be obtained by appending additional nodes to subgraph $\mathcal{S}$.

A consequence of this particular way to arrange the search space $\mathcal{M}$, with profound algorithmic implications, is the so called apriori property of pattern mining.

Proposition 8.2 (Apriori property) Let $\mathcal{S}, \mathcal{S}^{\prime} \in \mathcal{M}$ be two patterns such that $\mathcal{S}^{\prime}$ is a descendant of $\mathcal{S}$ in a pattern enumeration tree. Then, $r_{\mathcal{S}^{\prime}} \leq r_{\mathcal{S}}$, where $r_{\mathcal{S}}$ and $r_{\mathcal{S}^{\prime}}$ are the marginal counts of patterns $\mathcal{S}$ and $\mathcal{S}^{\prime}$ in $2 \times 2$ contingency tables computed from a dataset $\mathcal{D}=\left\{\left(x_{i}, y_{i}\right)\right\}_{i=1}^{n}$.

Proof By definition of pattern enumeration tree, if $\mathcal{S}^{\prime}$ is a descendant of $\mathcal{S}$ then $\mathcal{S} \subseteq \mathcal{S}^{\prime}$. Therefore, $\mathcal{S}^{\prime} \subseteq x$ implies $\mathcal{S} \subseteq x$ or, equivalently, $g_{\mathcal{S}^{\prime}}(x)=1$ implies $g_{\mathcal{S}}(x)=1$. Since $r_{\mathcal{S}}=\sum_{i=1}^{n} g_{\mathcal{S}}(x)$ and $r_{\mathcal{S}^{\prime}}=\sum_{i=1}^{n} g_{\mathcal{S}^{\prime}}(x)$ the result follows.

The apriori property is the formalization of an intuitive fact: If patterns are arranged in a tree guaranteeing that descendant nodes correspond to increasingly complex super-patterns, as we descend along the tree, patterns will be less frequently observed in a dataset $\mathcal{D}$. Despite its simplicity, the apriori property will play a central role in the development of a pruning criterion to avoid the need to explicitly enumerate all patterns in $\mathcal{M}$.

As shown in Line 2 of Algorithm 8.2, the routine tarone_spm begins by initialising the estimate $\hat{\delta}_{\text {tar }}$ to 1 , the largest value $\delta_{\text {tar }}$ could take, and the set of testable patterns at level $\hat{\delta}_{\text {tar }}$ to the empty set, $\widehat{\mathcal{M}}_{\text {test }}\left(\hat{\delta}_{\text {tar }}\right) \leftarrow \emptyset$. In order to compute $\delta_{\text {tar }}=$ $\max \left\{\delta|\delta| \mathcal{M}_{\text {test }}(\delta) \mid \leq \alpha\right\}$, candidate patterns $\mathcal{S} \in \mathcal{M}$ will be explored recursively by traversing the corresponding enumeration tree in a depth-first manner. The estimate $\hat{\delta}_{\text {tar }}$ and the corresponding set of testable patterns $\widehat{\mathcal{M}}_{\text {test }}\left(\hat{\delta}_{\text {tar }}\right)$ will be incrementally adjusted as patterns are enumerated in such a way that, at the end of the process, 
$\hat{\delta}_{\text {tar }}=\delta_{\text {tar }}$ and $\widehat{\mathcal{M}}_{\text {test }}\left(\hat{\delta}_{\text {tar }}\right)=\mathcal{M}_{\text {test }}\left(\delta_{\text {tar }}\right)$ holds. This enumeration procedure starts in Line 3 at the root of the tree, which by convention represents the empty pattern $\mathcal{S}=\emptyset .{ }^{9}$ Every time a pattern $\mathcal{S} \in \mathcal{M}$ is visited during the enumeration process, the following sequence of steps is performed.

First, in Line 7 the minimum attainable $P$-value $p_{\mathcal{S} \text {, min }}$ of the pattern $\mathcal{S}$ is evaluated based on the samples in the dataset $\mathcal{D}$. This will be detailed in Section 8.3.1. In the next line, the algorithm checks whether the pattern is testable at the current level $\hat{\delta}_{\text {tar }}$ (i.e., $p_{\mathcal{S}, \min } \leq \hat{\delta}_{\text {tar }}$ ) or not. If $\mathcal{S}$ is testable, it will be added to the estimate of the set of testable patterns $\widehat{\mathcal{M}}_{\text {test }}\left(\hat{\delta}_{\text {tar }}\right)$ in Line 9. Tarone's upper bound on the FWER will be evaluated next, using the current estimate $\widehat{\mathcal{M}}_{\text {test }}\left(\hat{\delta}_{\text {tar }}\right)$ of the set of testable patterns at level $\hat{\delta}_{\text {tar. }}$. In Line 11 , this value is subsequently used by the algorithm to check if the FWER condition $\hat{\delta}_{\text {tar }}\left|\mathcal{M}_{\text {test }}\left(\hat{\delta}_{\text {tar }}\right)\right|=\widehat{\operatorname{FWER}}\left(\hat{\delta}_{\text {tar }}\right) \leq \alpha$ is violated at level $\hat{\delta}_{\text {tar }}$. Note that, since the enumeration process is not yet completed, $\widehat{\mathcal{M}}_{\text {test }}\left(\hat{\delta}_{\text {tar }}\right) \subseteq \mathcal{M}_{\text {test }}\left(\hat{\delta}_{\text {tar }}\right)$ holds. As a consequence, the FWER approximation $\widetilde{F W E R}\left(\hat{\delta}_{\text {tar }}\right)$ evaluated in Line 10 satisfies $\widetilde{\operatorname{FWER}}\left(\hat{\delta}_{\text {tar }}\right)=\hat{\delta}_{\text {tar }}\left|\widehat{\mathcal{M}}_{\text {test }}\left(\hat{\delta}_{\text {tar }}\right)\right| \leq \hat{\delta}_{\text {tar }}\left|\mathcal{M}_{\text {test }}\left(\hat{\delta}_{\text {tar }}\right)\right|=\widehat{\operatorname{FWER}}\left(\hat{\delta}_{\text {tar }}\right)$. If $\widetilde{\text { FWER }}\left(\hat{\delta}_{\text {tar }}\right) \geq \alpha$, then it follows that $\widehat{\operatorname{FWER}}\left(\hat{\delta}_{\text {tar }}\right) \geq \alpha$ and we can immediately conclude that adjusted significance threshold $\hat{\delta}_{\text {tar }}$ is too large and violates the FWER condition for target FWER $\alpha$. Therefore, in that case, the current estimate of the adjusted significance threshold $\hat{\delta}_{\text {tar }}$ is decreased in Line 12 . This causes some patterns currently in $\widehat{\mathcal{M}}_{\text {test }}\left(\hat{\delta}_{\text {tar }}\right)$ to no longer be testable (Line 13), thereby reducing $\widehat{\text { FWER }}\left(\hat{\delta}_{\text {tar }}\right)$, which is re-evaluated in Line 14 . This process is repeated, incrementally reducing $\hat{\delta}_{\text {tar }}$, until the FWER condition is satisfied again, i.e., $\widetilde{F W E R}\left(\hat{\delta}_{\mathrm{tar}}\right) \leq \alpha$. Finally, the enumeration process continues recursively by visiting the children of the pattern $\mathcal{S}$ currently being processed (Lines 16-17). However, prior to that a pruning condition is evaluated in Line 15. This step, discussed in detail in Section 8.3.2, is essential to the computational feasibility of the algorithm. If the pruning condition applies, no descendant of pattern $\mathcal{S}$ will be testable and, hence, they do not need to be enumerated, drastically reducing computational complexity. As the algorithm enumerates patterns, $\hat{\delta}_{\text {tar }}$ progressively decreases, making more and more patterns become untestable and the pruning condition in Line 15 to be more stringent. Eventually, the algorithm converges when all patterns $\mathcal{S} \in \mathcal{M}$ that have not been pruned from the search space have been visited. At that point, $\hat{\delta}_{\operatorname{tar}}=\delta_{\mathrm{tar}}$ and $\widehat{\mathcal{M}}_{\text {test }}\left(\hat{\delta}_{\text {tar }}\right)=\mathcal{M}_{\text {test }}\left(\hat{\delta}_{\text {tar }}\right)$, allowing the exact value of $\delta_{\text {tar }}$ to be returned in Line 5 .

The framework described in Algorithms 8.1 and 8.2 can be readily applied to multiple instances of significant pattern mining and choices of test statistic to assess the significance of patterns. Two key steps of the algorithm that remain to be discussed are how to efficiently evaluate the minimum attainable $P$-value $p_{\mathcal{S} \text {, min }}$ and how to propose a valid pruning condition that results in a large reduction of the number of candidate patterns. In the remainder of this section, we discuss each of these issues for the two particular choices of the test statistic that were discussed in Section 8.2.2: Pearson's $\chi^{2}$ test and Fisher's exact test.

9 We define the empty pattern to occur in every input sample, i.e., $g_{\emptyset}(x)=1$ for all $x \in \mathcal{X}$. The empty pattern will thus never be statistically significant; its only purpose is to act as the starting point of the recursive enumeration. 


\subsubsection{Evaluating Tarone's Minimum Attainable $P$-value}

As described in Section 8.2.3, for a test statistic based on a $2 \times 2$ contingency table that treats margins $n_{1}$ and $r_{\mathcal{S}}$ as fixed quantities, such as Pearson's $\chi^{2}$ test and Fisher's exact test, the minimum attainable $P$-value $p_{\mathcal{S} \text {, min }}$ can be defined as:

$$
p_{\mathcal{S}, \min }=\min \left\{p_{\mathcal{S}}\left(a_{\mathcal{S}}^{\prime} \mid n, n_{1}, r_{\mathcal{S}}\right) \mid a_{\mathcal{S}}^{\prime} \in \llbracket a_{\mathcal{S}, \min }, a_{\mathcal{S}, \max } \rrbracket\right\},
$$

where $p_{\mathcal{S}}\left(a_{\mathcal{S}}^{\prime} \mid n, n_{1}, r_{\mathcal{S}}\right)$ is the $P$-value corresponding to a $2 \times 2$ contingency table with cell count $a_{\mathcal{S}}^{\prime}$, margins $n_{1}$ and $r_{\mathcal{S}}$, and sample size $n$.

Evaluating the minimum attainable $P$-value $p_{\mathcal{S} \text {, min }}$ of a pattern $\mathcal{S}$ based on the $n$ samples in the input dataset $\mathcal{D}$ is a key operation in Algorithm 8.2. In particular, the minimum attainable $P$-value needs to be evaluated for every single pattern $\mathcal{S} \in \mathcal{M}$ that is not eliminated by the pruning condition. In a typical execution of Algorithm 8.2, this can amount to billions of evaluations of $p_{\mathcal{S} \text {, min }}$, making the computation of $p_{\mathcal{S} \text {, min }}$ a critical point to maintain computational efficiency. Obtaining $p_{\mathcal{S} \text {, min }}$ by evaluating $p_{\mathcal{S}}\left(a_{\mathcal{S}}^{\prime} \mid n, n_{1}, r_{\mathcal{S}}\right)$ for each $a_{\mathcal{S}}^{\prime} \in \llbracket a_{\mathcal{S}, \min }, a_{\mathcal{S}, \max } \rrbracket$, as suggested by a naive application of Equation 8.12, might require $O(n) P$-value computations for each evaluation of $p_{\mathcal{S} \text {, min, }}$ an unacceptable computational overhead. In this section, we describe how $p_{\mathcal{S} \text {, min }}$ can be obtained with $O(1)$ complexity for Pearson's $\chi^{2}$ test and Fisher's exact test.

First, note that $p_{\mathcal{S}, \min }$ is a function of the number $r_{\mathcal{S}}$ of occurrences of pattern $\mathcal{S}$ in $\mathcal{D}$, the number $n_{1}$ of samples in $\mathcal{D}$ that belong to the positive class and the total sample size $n$. Since, for a given dataset $\mathcal{D}$, only $r_{\mathcal{S}}$ varies from one pattern to another, we will simply write $p_{\mathcal{S}, \min }\left(r_{\mathcal{S}}\right)$ to avoid notation clutter, leaving the dependence of $p_{\mathcal{S} \text {, min }}$ on $n_{1}$ and $n$ implicit.

In order to compute $p_{\mathcal{S}, \min }\left(r_{\mathcal{S}}\right)$, the minimizer $a_{\mathcal{S}}^{*}$ of $p_{\mathcal{S}}\left(a_{\mathcal{S}}^{\prime} \mid n, n_{1}, r_{\mathcal{S}}\right)$ in $\llbracket a_{\mathcal{S}, \min }$, $a_{\mathcal{S} \text {, max }} \rrbracket$ needs to be obtained. Due to the way the $P$-values are defined for Pearson's $\chi^{2}$ test (Equation 8.6) and Fisher's exact test (Equation 8.8), the minimizer $a_{\mathcal{S}}^{*}$ must be in the boundary of $\llbracket a_{\mathcal{S}, \min }, a_{\mathcal{S} \text {, max }} \rrbracket$, i.e., either $a_{\mathcal{S}}^{*}=a_{\mathcal{S}, \min }$ or $a_{\mathcal{S}}^{*}=a_{\mathcal{S} \text {, max }}$. All that remains to be shown is which of the two cases holds for each value of $r_{\mathcal{S}}$, leading to a closed-form of $p_{\mathcal{S}, \min }\left(r_{\mathcal{S}}\right)$ for each of the two test statistics.

Proposition 8.3 (Minimum attainable $P$-value for Pearson's $\chi^{2}$ test) Define $n_{a}=\min \left(n_{1}, n-n_{1}\right)$ and $n_{b}=\max \left(n_{1}, n-n_{1}\right)$. Then, the minimum attainable P-value for Pearson's $\chi^{2}$ test is given by:

$$
p_{\mathcal{S}, \min }\left(r_{\mathcal{S}}\right)=\left\{\begin{array}{lll}
1-F_{\chi_{1}^{2}}\left((n-1) \frac{n_{b}}{n_{a}} \frac{r_{\mathcal{S}}}{n-r_{\mathcal{S}}}\right), & \text { if } & 0 \leq r_{\mathcal{S}}<n_{a}, \\
1-F_{\chi_{1}^{2}}\left((n-1) \frac{n_{a}}{n_{b}} \frac{n-r_{\mathcal{S}}}{r_{\mathcal{S}}}\right), & \text { if } & n_{a} \leq r_{\mathcal{S}}<\frac{n}{2} \\
1-F_{\chi_{1}^{2}}\left((n-1) \frac{n_{a}}{n_{b}} \frac{r_{\mathcal{S}}}{n-r_{\mathcal{S}}}\right), & \text { if } & \frac{n}{2} \leq r_{\mathcal{S}}<n_{b} \\
1-F_{\chi_{1}^{2}}\left((n-1) \frac{n_{b}}{n_{a}} \frac{n-r_{\mathcal{S}}}{r_{\mathcal{S}}}\right), & \text { if } & n_{b} \leq r_{\mathcal{S}} \leq n .
\end{array}\right.
$$

Proof Let $T_{\max }\left(r_{\mathcal{S}}\right)$ be the maximum value of Pearson's $\chi^{2}$ test statistic for a $2 \times 2$ contingency table with sample size $n$ and margins $r_{\mathcal{S}}$ and $n_{1}$. As discussed above,

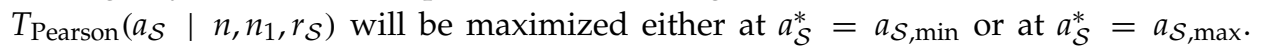


Hence:

$$
T_{\max }\left(r_{\mathcal{S}}\right)=\frac{\max \left(\left(a_{\mathcal{S}, \text { min }}-r_{\mathcal{S}} \frac{n_{1}}{n}\right)^{2},\left(a_{\mathcal{S}, \text { max }}-r_{\mathcal{S}} \frac{n_{1}}{n}\right)^{2}\right)}{\frac{r_{\mathcal{S}}}{n} \frac{n-r_{\mathcal{S}}}{n} \frac{n-n_{1}}{n-1} n_{1}} .
$$

Suppose $0 \leq r_{\mathcal{S}}<n_{a}$. Then, $a_{\mathcal{S}, \min }=0$ and $a_{\mathcal{S}, \max }=r_{\mathcal{S}}$, leading to:

$$
T_{\max }\left(r_{\mathcal{S}}\right)=(n-1) \frac{r_{\mathcal{S}}}{n-r_{\mathcal{S}}} \frac{\max ^{2}\left(n_{1}, n-n_{1}\right)}{n_{1}\left(n-n_{1}\right)}=(n-1) \frac{r_{\mathcal{S}}}{n-r_{\mathcal{S}}} \frac{n_{b}}{n_{a}} .
$$

Analogously, if $n_{b} \leq r_{\mathcal{S}} \leq n$, then $a_{\mathcal{S}, \min }=r_{\mathcal{S}}-\left(n-n_{1}\right)$ and $a_{\mathcal{S}, \max }=n_{1}$. Thus:

$$
T_{\max }\left(r_{\mathcal{S}}\right)=(n-1) \frac{n-r_{\mathcal{S}}}{r_{\mathcal{S}}} \frac{\max ^{2}\left(n_{1}, n-n_{1}\right)}{n_{1}\left(n-n_{1}\right)}=(n-1) \frac{n-r_{\mathcal{S}}}{r_{\mathcal{S}}} \frac{n_{b}}{n_{a}} .
$$

Finally, suppose $n_{a} \leq r_{\mathcal{S}}<n_{b}$. This case can be studied separately depending on whether $n_{1} \leq n-n_{1}$ or $n_{1}>n-n_{1}$.

Let $n_{1} \leq n-n_{1}$, then $a_{\mathcal{S}, \min }=0$ and $a_{\mathcal{S}, \max }=n_{1}$. This leads to:

$$
\begin{aligned}
T_{\max }\left(r_{\mathcal{S}}\right) & =(n-1) \frac{n_{1}}{n-n_{1}} \frac{\max ^{2}\left(r_{\mathcal{S}}, n-r_{\mathcal{S}}\right)}{r_{\mathcal{S}}\left(n-r_{\mathcal{S}}\right)}=(n-1) \frac{n_{a}}{n_{b}} \frac{\max ^{2}\left(r_{\mathcal{S}}, n-r_{\mathcal{S}}\right)}{r_{\mathcal{S}}\left(n-r_{\mathcal{S}}\right)} \\
& =\left\{\begin{array}{lll}
(n-1) \frac{n_{a}}{n_{b}} \frac{n-r_{\mathcal{S}}}{r_{\mathcal{S}}}, & \text { if } \quad n_{a} \leq r_{\mathcal{S}}<\frac{n}{2} \\
(n-1) \frac{n_{a}}{n_{b}} \frac{r_{\mathcal{S}}}{n-r_{\mathcal{S}}}, & \text { if } \quad \frac{n}{2} \leq r_{\mathcal{S}}<n_{b} .
\end{array}\right.
\end{aligned}
$$

If $n_{1}>n-n_{1}$, then $a_{\mathcal{S}, \min }=r_{\mathcal{S}}-\left(n-n_{1}\right)$ and $a_{\mathcal{S}, \max }=r_{\mathcal{S}}$. Therefore:

$$
\begin{aligned}
T_{\max }\left(r_{\mathcal{S}}\right) & =(n-1) \frac{n-n_{1}}{n_{1}} \frac{\max ^{2}\left(r_{\mathcal{S}}, n-r_{\mathcal{S}}\right)}{r_{\mathcal{S}}\left(n-r_{\mathcal{S}}\right)}=(n-1) \frac{n_{a}}{n_{b}} \frac{\max ^{2}\left(r_{\mathcal{S}}, n-r_{\mathcal{S}}\right)}{r_{\mathcal{S}}\left(n-r_{\mathcal{S}}\right)} \\
& = \begin{cases}(n-1) \frac{n_{a}}{n_{b}} \frac{n-r_{\mathcal{S}}}{r_{\mathcal{S}}}, & \text { if } \quad n_{a} \leq r_{\mathcal{S}}<\frac{n}{2}, \\
(n-1) \frac{n_{a}}{n_{b}} \frac{r_{\mathcal{S}}}{n-r_{\mathcal{S}}}, & \text { if } \quad \frac{n}{2} \leq r_{\mathcal{S}}<n_{b} .\end{cases}
\end{aligned}
$$

Since $p_{\text {Pearson }}\left(a_{\mathcal{S}} \mid n, n_{1}, r_{\mathcal{S}}\right)=1-F_{\chi_{1}^{2}}\left(T_{\text {Pearson }}\left(a_{\mathcal{S}} \mid n, n_{1}, r_{\mathcal{S}}\right)\right)$, this concludes the proof.

Proposition 8.4 (Minimum attainable $P$-value for Fisher's exact test) Define $n_{a}=\min \left(n_{1}, n-n_{1}\right)$ and $n_{b}=\max \left(n_{1}, n-n_{1}\right)$. Then, the minimum attainable P-value for Fisher's exact test is given by:

$$
p_{\mathcal{S}, \min }\left(r_{\mathcal{S}}\right)=\left\{\begin{array}{lll}
\left(\begin{array}{c}
n_{a} \\
r_{\mathcal{S}}
\end{array}\right) /\left(\begin{array}{c}
n \\
r_{\mathcal{S}}
\end{array}\right), & \text { if } & 0 \leq r_{\mathcal{S}}<n_{a}, \\
\left(\begin{array}{c}
n_{b} \\
n-r_{\mathcal{S}}
\end{array}\right) /\left(\begin{array}{c}
n \\
r_{\mathcal{S}}
\end{array}\right), & \text { if } & n_{a} \leq r_{\mathcal{S}}<\frac{n}{2} \\
\left(\begin{array}{c}
n \\
r_{\mathcal{S}}
\end{array}\right) /\left(\begin{array}{c}
n \\
r_{\mathcal{S}}
\end{array}\right), & \text { if } & \frac{n}{2} \leq r_{\mathcal{S}}<n_{b}, \\
\left(\begin{array}{c}
n_{a} \\
n-r_{\mathcal{S}}
\end{array}\right) /\left(\begin{array}{c}
n \\
r_{\mathcal{S}}
\end{array}\right), & \text { if } & n_{b} \leq r_{\mathcal{S}} \leq n .
\end{array}\right.
$$

Proof Analogously to the proof of Proposition 8.3, $p_{\text {fisher }}\left(a_{\mathcal{S}} \mid n, n_{1}, r_{\mathcal{S}}\right)$ is minimized

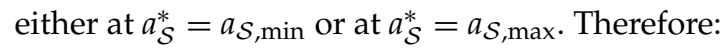

$$
\begin{aligned}
p_{\mathcal{S}, \min }\left(r_{\mathcal{S}}\right) & =\min \left(\operatorname{Hypergeom}\left(a_{\mathcal{S}, \min } \mid n, n_{1}, r_{\mathcal{S}}\right), \operatorname{Hypergeom}\left(a_{\mathcal{S}, \max } \mid n, n_{1}, r_{\mathcal{S}}\right)\right) \\
& =\frac{\min \left(\left(\begin{array}{c}
n_{1} \\
a_{\mathcal{S}, \min }
\end{array}\right)\left(\begin{array}{c}
n-n_{1} \\
r_{\mathcal{S}}-a_{\mathcal{S}, \min }
\end{array}\right)\left(\begin{array}{c}
n_{1} \\
a_{\mathcal{S}, \max }
\end{array}\right)\left(\begin{array}{c}
n-n_{1} \\
r_{\mathcal{S}}-a_{\mathcal{S}, \max }
\end{array}\right)\right)}{\left(\begin{array}{c}
n \\
r_{\mathcal{S}}
\end{array}\right)}
\end{aligned}
$$


The problem will be decomposed in three cases, as done for the derivation of the closed-form expression of the minimum attainable $P$-value for Pearson's $\chi^{2}$ test.

Let $0 \leq r_{\mathcal{S}}<n_{a}$, leading to $a_{\mathcal{S}, \min }=0$ and $a_{\mathcal{S}, \max }=r_{\mathcal{S}}$. Then:

$$
p_{\mathcal{S}, \min }\left(r_{\mathcal{S}}\right)=\frac{\min \left(\left(\begin{array}{c}
n-n_{1} \\
r_{\mathcal{S}}
\end{array}\right),\left(\begin{array}{c}
n_{1} \\
r_{\mathcal{S}}
\end{array}\right)\right)}{\left(\begin{array}{c}
n \\
r_{\mathcal{S}}
\end{array}\right)}=\frac{\left(\begin{array}{c}
n_{a} \\
r_{\mathcal{S}}
\end{array}\right)}{\left(\begin{array}{c}
n \\
r_{\mathcal{S}}
\end{array}\right)} .
$$

Similarly, if $n_{b} \leq r_{\mathcal{S}} \leq n$ then $a_{\mathcal{S}, \min }=r_{\mathcal{S}}-\left(n-n_{1}\right)$ and $a_{\mathcal{S}, \max }=n_{1}$. Thus:

$$
\begin{aligned}
p_{\mathcal{S}, \min }\left(r_{\mathcal{S}}\right) & =\frac{\min \left(\left(\begin{array}{c}
n_{1} \\
r_{\mathcal{S}}-\left(n-n_{1}\right)
\end{array}\right),\left(\begin{array}{c}
n-n_{1} \\
r_{\mathcal{S}}-n_{1}
\end{array}\right)\right)}{\left(\begin{array}{c}
n \\
r_{\mathcal{S}}
\end{array}\right)}=\frac{\min \left(\left(\begin{array}{c}
n_{1} \\
n-r_{\mathcal{S}}
\end{array}\right),\left(\begin{array}{c}
n-n_{1} \\
n-r_{\mathcal{S}}
\end{array}\right)\right)}{\left(\begin{array}{c}
n \\
r_{\mathcal{S}}
\end{array}\right)} \\
& =\frac{\left(\begin{array}{c}
n_{a} \\
n-r_{\mathcal{S}}
\end{array}\right)}{\left(\begin{array}{c}
n \\
r_{\mathcal{S}}
\end{array}\right)},
\end{aligned}
$$

where the second step follows from $\left(\begin{array}{l}n \\ k\end{array}\right)=\left(\begin{array}{c}n \\ n-k\end{array}\right)$.

Let $n_{a} \leq r_{\mathcal{S}}<n_{b}$ and $n_{1} \leq n-n_{1}$, so that $a_{\mathcal{S}, \min }=0$ and $a_{\mathcal{S}, \max }=n_{1}$. Hence:

$$
\begin{aligned}
p_{\mathcal{S}, \min }\left(r_{\mathcal{S}}\right) & =\frac{\min \left(\left(\begin{array}{c}
n-n_{1} \\
r_{\mathcal{S}}
\end{array}\right),\left(\begin{array}{c}
n-n_{1} \\
n-r_{\mathcal{S}}
\end{array}\right)\right)}{\left(\begin{array}{c}
n \\
r_{\mathcal{S}}
\end{array}\right)} \\
& = \begin{cases}\frac{\left(\begin{array}{c}
n_{b} \\
n-r_{\mathcal{S}}
\end{array}\right)}{\left(\begin{array}{l}
n \\
r_{\mathcal{S}}
\end{array}\right)}, & \text { if } \quad n_{a} \leq r_{\mathcal{S}}<\frac{n}{2}, \\
\frac{\left(\begin{array}{c}
n_{\mathcal{S}} \\
r_{\mathcal{S}}
\end{array}\right.}{\left(\begin{array}{c}
n \\
r_{\mathcal{S}}
\end{array}\right)}, & \text { if } \quad \frac{n}{2} \leq r_{\mathcal{S}}<n_{b} .\end{cases}
\end{aligned}
$$

Alternatively, if $n_{a} \leq r_{\mathcal{S}}<n_{b}$ and $n_{1}>n-n_{1}$, then $a_{\mathcal{S}, \min }=r_{\mathcal{S}}-\left(n-n_{1}\right)$ and $a_{\mathcal{S}, \max }=r_{\mathcal{S}}$, leading to:

$$
\begin{aligned}
p_{\mathcal{S}, \min }\left(r_{\mathcal{S}}\right) & =\frac{\min \left(\left(\begin{array}{c}
n_{1} \\
n-r_{\mathcal{S}}
\end{array}\right),\left(\begin{array}{c}
n_{1} \\
r_{\mathcal{S}}
\end{array}\right)\right)}{\left(\begin{array}{c}
n \\
r_{\mathcal{S}}
\end{array}\right)} \\
& = \begin{cases}\frac{\left(\begin{array}{c}
n_{b} \\
n-r_{\mathcal{S}}
\end{array}\right)}{\left(\begin{array}{c}
n \\
r_{\mathcal{S}}
\end{array}\right)}, & \text { if } \quad n_{a} \leq r_{\mathcal{S}}<\frac{n}{2}, \\
\left(\begin{array}{l}
n_{b} \\
r_{\mathcal{S}}
\end{array}\right) & \text { if } \quad \frac{n}{2} \leq r_{\mathcal{S}}<n_{b}, \\
\left(\begin{array}{l}
n \\
r_{\mathcal{S}}
\end{array}\right)\end{cases}
\end{aligned}
$$

where again the identity $\left(\begin{array}{l}n \\ k\end{array}\right)=\left(\begin{array}{c}n \\ n-k\end{array}\right)$ was used. This concludes the proof.

Figure 8.4 illustrates the behavior of $p_{\mathcal{S} \text {, min }}$ for Pearson's $\chi^{2}$ test (blue) and Fisher's exact test (orange) as the number $r_{\mathcal{S}}$ of occurrences of pattern $\mathcal{S}$ in a dataset $\mathcal{D}$ varies. In Figure 8.4 (a), the sample size is $n=60$ and the number of samples in the positive class is $n_{1}=15$, whereas Figure 8.4 (b) shows an example with a balanced class ratio, i.e., $n=60$ and $n_{1}=30$. First, $p_{\mathcal{S}, \min }$ shows a qualitatively identical behaviour for both test statistics, as expected. For fixed $n_{1}$ and $n, p_{\mathcal{S} \text {, min }}$ as a function of $r_{\mathcal{S}}$ is symmetric around $r_{\mathcal{S}}=n / 2$ and has minima at $r_{\mathcal{S}}=n_{a}=\min \left(n_{1}, n-n_{1}\right)$ and $r_{\mathcal{S}}=n_{b}=\max \left(n_{1}, n-n_{1}\right)$, as evidenced by the closed-form expressions derived in Propositions 8.3 and 8.4. The key insight to be derived from Figure 8.4 is that $p_{\mathcal{S}, \min }$ is large, indicating lower potential to result in a statistically significant association, whenever $r_{\mathcal{S}}$ is small or $r_{\mathcal{S}}$ is large. 


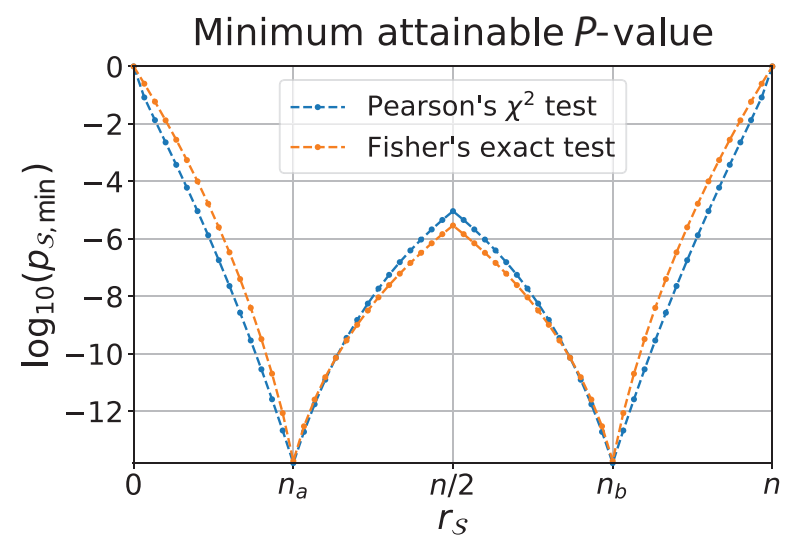

(a)

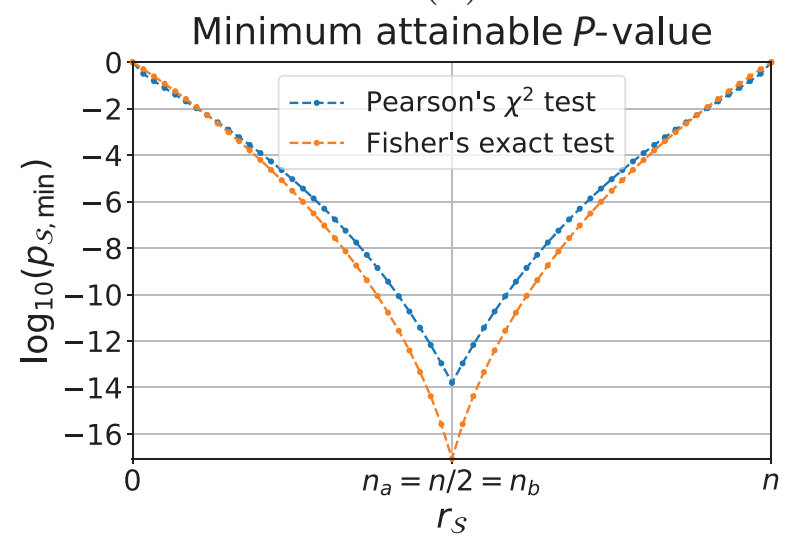

(b)

Figure 8.4: Minimum attainable $P$-value $p_{\mathcal{S} \text {, } \min }$ as a function of $r_{\mathcal{S}}$, the number of occurrences of pattern $\mathcal{S}$ in a dataset $\mathcal{D}$, for Pearson's $\chi^{2}$ test (blue) and Fisher's exact test (orange). The number of samples in the positive class $n_{1}$ and the sample size $n$ are $n_{1}=15$, $n=60$ in (a) and $n_{1}=30, n=60$ in (b), respectively.

This is further illustrated in Figure 8.5, which indicates how this relates to the concept of testability at a certain adjusted significance threshold $\delta$. As a direct consequence of the functional form of $p_{\mathcal{S} \text {, min }}$, for fixed margin $n_{1}$, sample size $n$ and adjusted significance threshold $\delta$, there exists a value $r_{\mathcal{S}, \min }(\delta)$ such that patterns $\mathcal{S}$ with $r_{\mathcal{S}}<r_{\mathcal{S}, \min }(\delta)$ or $r_{\mathcal{S}}>n-r_{\mathcal{S}, \min }(\delta)$ are untestable at level $\delta$, while those with $\llbracket r_{\mathcal{S}, \min }(\delta), n-r_{\mathcal{S}, \min }(\delta) \rrbracket$ are testable. ${ }^{10}$ This result formalizes the intuition that patterns $\mathcal{S}$ with either too small $r_{\mathcal{S}}$ or too large $r_{\mathcal{S}}$, i.e. patterns $\mathcal{S}$ that are either too rare or too common in $\mathcal{D}$, cannot be

10 To be precise, if $\delta$ is sufficiently small, the range of values of $r_{\mathcal{S}}$ that lead to pattern $\mathcal{S}$ being testable can become the union of two disjoint intervals. However, this poses no practical algorithmic or statistical issues. Moreover, this situation is uncommon in practice, as it corresponds to values of $\delta$ that are too small to be of practical relevance in most applications. 


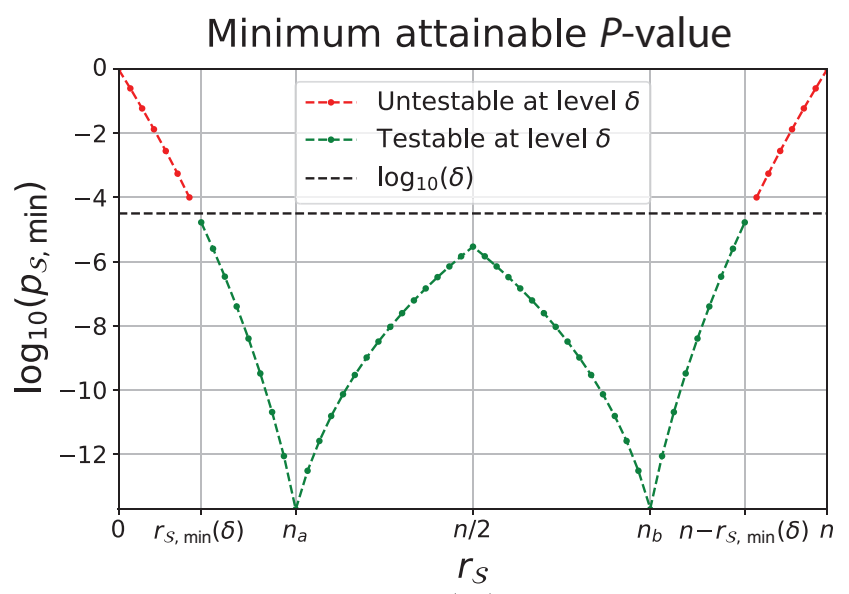

(a)

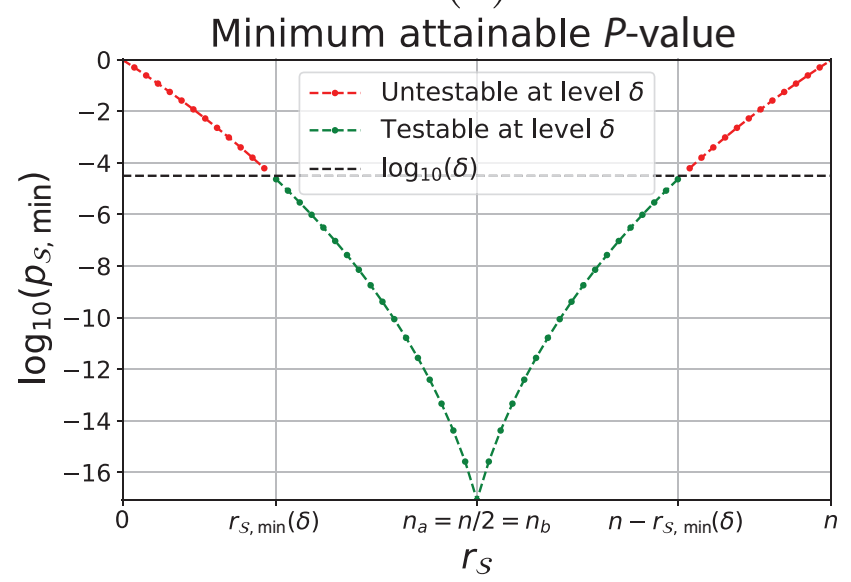

(b)

Figure 8.5: Illustration of the concept of testability at level $\delta=10^{-4.5}$ when Fisher's exact test is the test statistic of choice for two $2 \times 2$ contingency tables that differ in the class ratios: (a) unbalanced, with $n_{1}=15, n=60$ and (b) balanced, with $n_{1}=30, n=60$. Values of $r_{\mathcal{S}}$ in the range $\llbracket r_{\mathcal{S}, \min }(\delta), n-r_{\mathcal{S}, \min }(\delta) \rrbracket$ lead to pattern $\mathcal{S}$ being testable at level $\delta$ (green), while $r_{\mathcal{S}}<r_{\mathcal{S}, \min }(\delta)$ or $r_{\mathcal{S}}>n-r_{\mathcal{S}, \min }(\delta)$ imply that pattern $\mathcal{S}$ is untestable at level $\delta$ (red).

statistically significant. Tarone's method can therefore be understood as a statistically principled way to turn this intuition into a filtering criterion to reduce the number of patterns that contribute to the multiple hypothesis testing problem. Most importantly, unlike other alternative approaches, Tarone's method does not use an ad-hoc threshold to filter patterns according to their frequency. It rather learns the threshold in a datadriven manner. This allows Tarone's method to guarantee the filtering of all patterns that have no chance of resulting in a statistically significant association at level $\delta$, regardless of the actual realizations of the class labels, while keeping all patterns that could result in an association. 


\subsubsection{Designing a Pruning Condition}

Even if the minimum attainable $P$-value $p_{\mathcal{S} \text {, min }}$ can be evaluated with $O(1)$ complexity, evaluating $p_{\mathcal{S}, \min }$ for all candidate patterns $\mathcal{M}$ is typically computationally unfeasible. Algorithm 8.2 relies on the existence of a pruning condition, that is, a way to test if descendants $\mathcal{S}^{\prime} \in$ Children $(\mathcal{S})$ of a pattern $\mathcal{S}$ in the enumeration tree are testable using only information available in the $2 \times 2$ contingency table of pattern $\mathcal{S}$. In this section, we show how the specific functional form of $p_{\mathcal{S} \text {, min }}$ for Pearson's $\chi^{2}$ test and Fisher's exact test, combined with the apriori property of pattern mining (Proposition 8.2), leads to the simple yet highly effective pruning criterion summarized in the following proposition:

Proposition 8.5 (Pruning criterion for Pearson's $\chi^{2}$ test and Fisher's exact test) Let $\mathcal{S} \in \mathcal{M}$ be a pattern satisfying:

i. $p_{\mathcal{S}, \min }>\hat{\delta}_{\text {tar }}$, i.e., $\mathcal{S}$ is untestable at level $\hat{\delta}_{\text {tar }}$,

ii. $\quad r_{\mathcal{S}} \leq n_{a}$, with $n_{a}=\min \left(n_{1}, n-n_{1}\right)$.

Then, $p_{\mathcal{S}^{\prime} \text {,min }}>\hat{\delta}_{\text {tar }} \geq \delta_{\text {tar }}$ for all $\mathcal{S}^{\prime} \in \operatorname{Children}(\mathcal{S})$. Hence, all descendants of pattern $\mathcal{S}$ can be pruned from the enumeration tree.

In conclusion, pruning_condition $\left(\mathcal{S}, \hat{\delta}_{\text {tar }}\right)$ in Line 15 of Algorithm 8.2 is true if and only if $r_{\mathcal{S}} \leq n_{a}$ and $p_{\mathcal{S}, \min }>\hat{\delta}_{\text {tar }}$.

Proof First, for fixed sample size $n$ and number $n_{1}$ of samples in the positive class, the minimum attainable $P$-value $p_{\mathcal{S} \text {, min }}$ is a monotonically decreasing function of $r_{\mathcal{S}}$ in the range $r_{\mathcal{S}} \in \llbracket 0, n_{a} \rrbracket$ for both Pearson's $\chi^{2}$ test and Fisher's exact test, i.e., $r_{\mathcal{S}^{\prime}} \leq r_{\mathcal{S}} \leq n_{a}$ implies $p_{\mathcal{S} \text {, } \min }\left(r_{\mathcal{S}^{\prime}}\right) \geq p_{\mathcal{S} \text {, } \min }\left(r_{\mathcal{S}}\right)$. This property, which does not necessarily hold for all test statistics based on contingency tables, can be readily verified from the specific functional form of $p_{\mathcal{S}, \min }$ in the range $r_{\mathcal{S}} \in \llbracket 0, n_{a} \rrbracket: p_{\mathcal{S}, \min }\left(r_{\mathcal{S}}\right)=1-F_{\chi_{1}^{2}}\left((n-1) \frac{n_{b}}{n_{a}} \frac{r_{\mathcal{S}}}{n-r_{\mathcal{S}}}\right)$ for Pearson's $\chi^{2}$ test and $p_{\mathcal{S}, \min }\left(r_{\mathcal{S}}\right)=\left(\begin{array}{c}n_{a} \\ r_{\mathcal{S}}\end{array}\right) /\left(\begin{array}{c}n \\ r_{\mathcal{S}}\end{array}\right)$ for Fisher's exact test. Intuitively, this means that in the range $r_{\mathcal{S}} \in \llbracket 0, n_{a} \rrbracket$, as the pattern $\mathcal{S}$ becomes less rare, the minimum attainable $P$-value decreases, thereby increasing the potential of pattern $\mathcal{S}$ to be statistically significant. Secondly, due to the apriori property, if $\mathcal{S}^{\prime} \in \operatorname{Children}(\mathcal{S})$ then $r_{\mathcal{S}^{\prime}} \leq r_{\mathcal{S}}$.

Combining both facts, if $r_{\mathcal{S}} \leq n_{a}$ then $p_{\mathcal{S}^{\prime} \text {,min }} \geq p_{\mathcal{S} \text {, min }}$ for all descendants $\mathcal{S}^{\prime}$ of $\mathcal{S}$ in the enumeration tree. Therefore, $p_{\mathcal{S}, \min }>\hat{\delta}_{\text {tar }}$ implies that $p_{\mathcal{S}^{\prime} \text {,min }}>\hat{\delta}_{\text {tar }}$ for all $\mathcal{S}^{\prime} \in \operatorname{Children}(\mathcal{S})$. Since $\hat{\delta}_{\mathrm{tar}} \geq \delta_{\mathrm{tar}}$ at any point during the execution of Algorithm 8.2, this proves the result.

Search space pruning according to the criterion presented in Proposition 8.5 can only occur for patterns $\mathcal{S} \in \mathcal{M}$ satisfying $r_{\mathcal{S}} \leq n_{a}$. In practice, a large proportion of all candidate patterns in the search space $\mathcal{M}$ are sufficiently rare for this condition to apply. When this happens, Proposition 8.5 simply states that descendants $\mathcal{S}^{\prime} \in$ Children $(\mathcal{S})$ of an untestable pattern $\mathcal{S}$ will also be untestable. As illustrated in Figure 8.6, this can lead to an enormous reduction in computational complexity, allowing one to compute $\delta_{\text {tar }}$ and (exactly) retrieve all testable patterns $\mathcal{M}_{\text {test }}\left(\delta_{\text {tar }}\right)$ by enumerating only a small subset of all candidate patterns in the search space. 


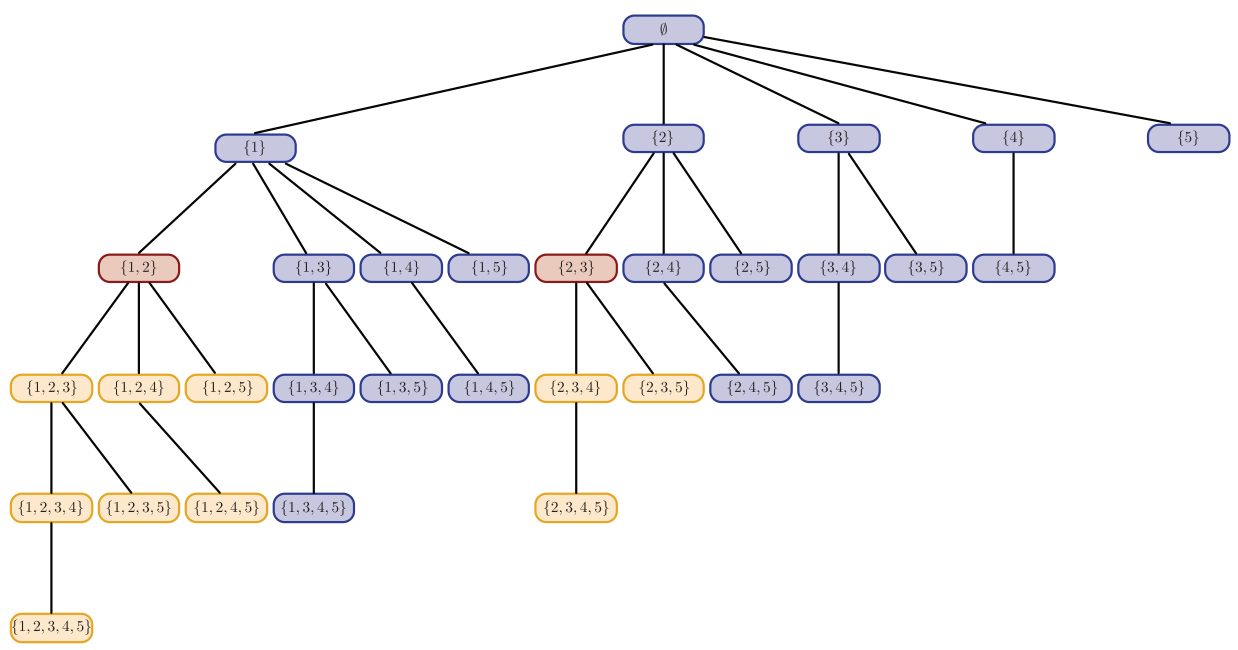

Figure 8.6: Illustration of the effect of search space pruning in a significant itemset mining problem with $p=5$ features. Suppose that patterns $\mathcal{S}_{1}$ and $\mathcal{S}_{2}$ (highlighted in red) satisfy the conditions of Proposition 8.5, i.e., $r_{\mathcal{S}_{1}} \leq n_{a}, r_{\mathcal{S}_{2}} \leq n_{a}$ and $p_{\mathcal{S}_{1}, \min }>\hat{\delta}_{\text {tar }}, p_{\mathcal{S}_{2}, \min }>\hat{\delta}_{\text {tar }}$. Then, all their descendants (highlighted in orange) can be pruned from the search space, drastically reducing the number of candidate patterns that need to be enumerated.

\subsubsection{Implementation Considerations}

We will conclude this section by discussing some key design considerations that need to be addressed when implementing a significant pattern mining algorithm.

The first and perhaps most important issue is the choice of algorithm to traverse the enumeration tree containing all patterns in the search space $\mathcal{M}$. Due to the enormous number of candidate patterns in the enumeration tree, the resulting efficiency of the traversal algorithm can greatly depend on the specific strategy and data structures used to perform the enumeration. While this topic is outside the scope of this chapter, the design of efficient algorithms to enumerate patterns has been widely studied by the data mining community, and many highly optimized methods are readily available for itemset mining (e.g., [40, 41, 42], see [43] for a review) and subgraph mining (e.g., [44, 45], see [46] for a review).

In Line 12 of Algorithm 8.2, the estimate $\hat{\delta}_{\text {tar }}$ of the adjusted significance threshold is decreased after the current value has been found to violate the FWER condition. There are multiple ways to implement this step. In theory, using either $\hat{\delta}_{\text {tar }} \leftarrow \hat{\delta}_{\text {tar }}-\Delta$ or $\hat{\delta}_{\text {tar }} \leftarrow 10^{-\Delta} \hat{\delta}_{\text {tar }}$, for sufficiently small $\Delta$, are both valid strategies. A more efficient alternative is to define the sequence of candidate values for $\hat{\delta}_{\text {tar }}$ by sorting, in descending order, the $\left\lfloor\frac{n}{2}\right\rfloor+1$ different values the minimum attainable $P$-value $p_{\mathcal{S} \text {, min }}$ can take. ${ }^{11}$ Each time $\hat{\delta}_{\text {tar }}$ needs to be decreased, it is set to the next element of the sequence. This is optimal in the sense that it provides the minimum step size necessary to decrease the FWER approximation $\widetilde{\text { FWER }}\left(\hat{\delta}_{\text {tar }}\right)$.

11 This follows from the fact that, for fixed $n_{1}$ and $n, p_{\mathcal{S}, \min }\left(r_{\mathcal{S}}\right)$ as a function of $r_{\mathcal{S}}$ is symmetric around $n / 2$. 
Another aspect worth considering is how to store in memory the set $\widehat{\mathcal{M}}_{\text {test }}\left(\hat{\delta}_{\text {tar }}\right)$ of testable patterns at level $\hat{\delta}_{\text {tar }}$ during the execution of Algorithm 8.2. Typically, this set can be rather large, containing hundreds of millions or even billions of patterns. Moreover, this could cause the execution of Line 13 in Algorithm 8.2 to become a computational bottleneck, since in principle the set $\widehat{\mathcal{M}}_{\text {test }}\left(\hat{\delta}_{\text {tar }}\right)$ of testable patterns needs to be inspected to remove all patterns which become untestable after having decreased $\hat{\delta}_{\text {tar }}$ in the previous line. The key insight to circumvent this problem is that, in order to approximate the FWER using Tarone's method, only the total number of testable patterns $\left|\widehat{\mathcal{M}}_{\text {test }}\left(\hat{\delta}_{\text {tar }}\right)\right|$ is needed, not the patterns themselves. Let $\mathcal{P}_{\text {min }}=$ $\left\{p_{\mathcal{S}, \min }\left(r_{\mathcal{S}}\right) \mid r_{\mathcal{S}} \in \llbracket 0,\left\lfloor\frac{n}{2}\right\rfloor \rrbracket\right\}$ be the set of $\left\lfloor\frac{n}{2}\right\rfloor+1$ distinct values that $p_{\mathcal{S} \text {, min }}$ can take for a given sample size $n$. Rather than explicitly storing $\widehat{\mathcal{M}}_{\text {test }}\left(\hat{\delta}_{\text {tar }}\right)$, an alternative strategy is to store the number $c\left(p_{\mathcal{S}, \text { min }}\right)$ of patterns enumerated so far that have minimum attainable $P$-value equal to $p_{\mathcal{S} \text {, min }}$, for each of the $\left\lfloor\frac{n}{2}\right\rfloor+1$ different values $p_{\mathcal{S} \text {, min }} \in \mathcal{P}_{\text {min }}$. For any given $\hat{\delta}_{\text {tar }},\left|\widehat{\mathcal{M}}_{\text {test }}\left(\hat{\delta}_{\text {tar }}\right)\right|$ can be computed as

$$
\left|\widehat{\mathcal{M}}_{\text {test }}\left(\hat{\delta}_{\text {tar }}\right)\right|=\sum_{p_{\mathcal{S}, \text { min }} \in \mathcal{P}_{\min } \mid p_{\mathcal{S}, \min } \leq \hat{\delta}_{\text {tar }}} c\left(p_{\mathcal{S}, \text { min }}\right),
$$

where the summation includes at most $\left\lfloor\frac{n}{2}\right\rfloor+1$ counts. This eliminates the need to store the set $\widehat{\mathcal{M}}_{\text {test }}\left(\hat{\delta}_{\text {tar }}\right)$ of testable patterns in memory and allows to execute Line 13 of Algorithm 8.2 with $O(1)$ complexity. Once $\delta_{\text {tar }}$ has been obtained, in Line 2 of Algorithm 8.1 , the set of testable patterns $\mathcal{M}_{\text {test }}\left(\delta_{\text {tar }}\right)$ is needed in order to find those patterns that are statistically significant. To do so, the enumeration process can be repeated, starting again at the root of the enumeration tree, but with fixed $\hat{\delta}_{\mathrm{tar}}=\delta_{\mathrm{tar}}$. As patterns are enumerated, the $P$-values $p_{\mathcal{S}}$ of patterns testable at level $\delta_{\text {tar }}$ are computed, and those which are deemed statistically significant are written to an output file. This is summarized in Algorithm 8.3. While this approach requires enumerating patterns twice, therefore approximately doubling runtime, it greatly reduces memory usage. Hence, it is the preferred implementation choice in most situations.

Finally, a strategy that might be useful in some cases is to precompute the $n$ values of $p_{\mathcal{S}, \min }\left(r_{\mathcal{S}}\right)$ and store them as a look-up table. While this requires $O(n)$ additional

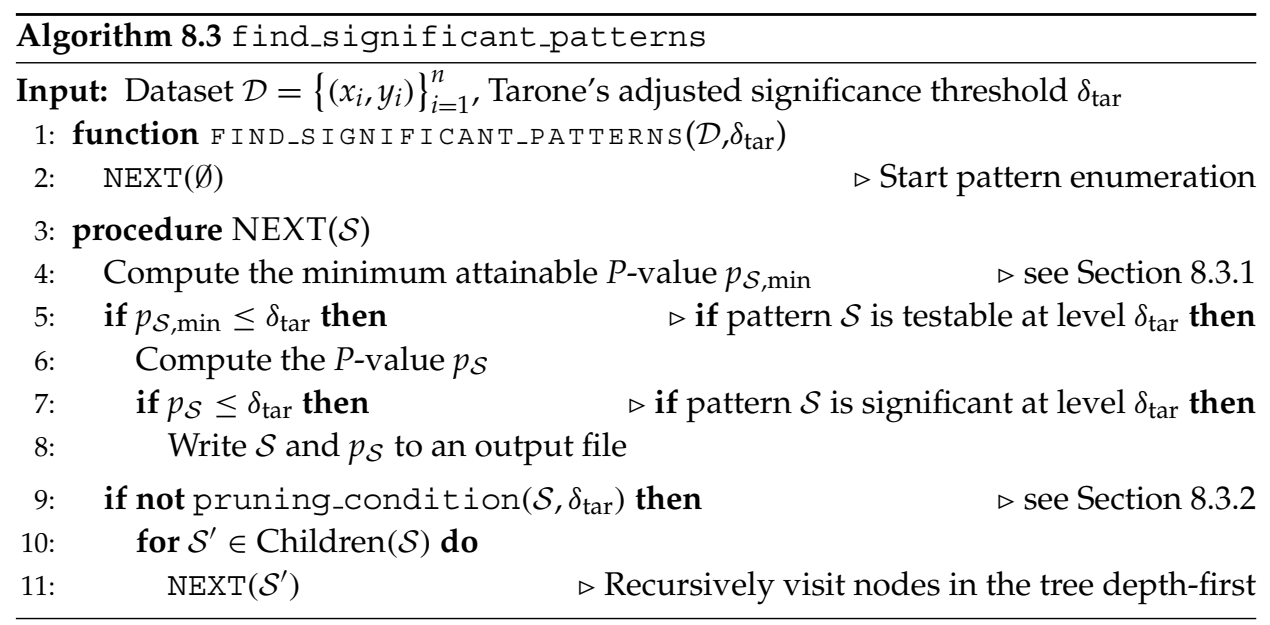


memory, it will further speed-up the evaluation of the minimum attainable $P$-values, which is one of the most repeated operations in Algorithm 8.2. As a consequence, in some situations, it might be a desirable trade-off of slightly increased memory usage for reduced runtime.

\subsection{Accounting for the Redundancy Between Patterns}

One of the defining characteristics of significant pattern mining is the daunting size of the search space of candidate patterns $\mathcal{M}$. As it has been extensively discussed in this chapter, this leads to two fundamental challenges: (1) the statistical challenge of dealing with an extreme instance of the multiple hypothesis testing problem, and (2) the computational challenge of efficiently exploring this vast search space $\mathcal{M}$. In Section 8.2.3, Tarone's improved Bonferroni correction for discrete data was introduced as a way to achieve FWER control while maintaining sufficient statistical power to discover truly associated patterns. Next, in Section 8.3, it was shown how Tarone's method can be combined with pattern mining techniques. This led to an algorithm that can explore all candidate patterns in $\mathcal{M}$ in a computationally efficient manner and that exhibits a considerable amount of statistical power despite guaranteeing FWER control. However, this approach still has key limitations, providing opportunities to develop new algorithms and to further improve the state-of-the-art in significant pattern mining.

In particular, another defining characteristic of significant pattern mining, which has been overlooked in this chapter so far, is the fact that the search space of candidate patterns $\mathcal{M}$ is not only extremely large, but also harbors non-trivial redundancies among the many patterns $\mathcal{M}$ contains.

One of the main sources of redundancy are subset/superset relationships between patterns. If a pattern $\mathcal{S}^{\prime}$ contains another pattern $\mathcal{S}$, the random variables $G_{\mathcal{S}}(X)$ and $G_{\mathcal{S}^{\prime}}(X)$ that indicate the occurrence of patterns $\mathcal{S}$ and $\mathcal{S}^{\prime}$ in an input sample $X$ satisfy $G_{\mathcal{S}}(X)=0 \Rightarrow G_{\mathcal{S}^{\prime}}(X)=0$ or, equivalently, $G_{\mathcal{S}^{\prime}}(X)=1 \Rightarrow G_{\mathcal{S}}(X)=1$. Therefore, $G_{\mathcal{S}}(X)$ and $G_{\mathcal{S}^{\prime}}(X)$ are statistically dependent, ${ }^{12}$ being mutually redundant to some degree. The strength of this dependency will depend, in general, on how frequently the "difference pattern" $\mathcal{S}^{\prime} \backslash \mathcal{S}$ occurs in an input sample X. The more common it is, the more frequently $\mathcal{S}$ and $\mathcal{S}^{\prime}$ will co-occur, leading to a stronger association between $G_{\mathcal{S}}(X)$ and $G_{\mathcal{S}^{\prime}}(X)$ and, therefore, a larger statistical redundancy between $\mathcal{S}$ and $\mathcal{S}^{\prime}$. This phenomenon is illustrated in Figure 8.7, which represents a toy significant itemset mining problem with $p=10$ features and $n=12$ samples. Given a high-order feature interaction $\mathcal{S}=\{2,9,10\}$, suppose that another high-order feature interaction $\mathcal{S}^{\prime}=$ $\mathcal{S} \cup\{7\}$ is formed by adding an additional feature to $\mathcal{S}$. Then, knowing the value of $g_{\mathcal{S}}\left(x_{i}\right)$ alone is sufficient to know the value of $g_{\mathcal{S}^{\prime}}\left(x_{i}\right)$ for half of the samples $\left\{x_{i}\right\}_{i=1}^{12}$ in the dataset. In fact, this holds true for any $\mathcal{S}^{\prime} \subset \mathcal{S}$, regardless of the additional features $\mathcal{S}^{\prime} \backslash \mathcal{S}$ being added to the high-order feature interaction. Consequently, $G_{\mathcal{S}}(X)$ is statistically dependent of $G_{\mathcal{S}^{\prime}}(X)$, for any $\mathcal{S}^{\prime} \subset \mathcal{S}$.

12 The apriori property of pattern mining, discussed in Proposition 8.2, can also be used to show that $G_{\mathcal{S}}(X)$ and $G_{\mathcal{S}^{\prime}}(X)$ are statistically dependent for $\mathcal{S}^{\prime} \subset \mathcal{S}$. 


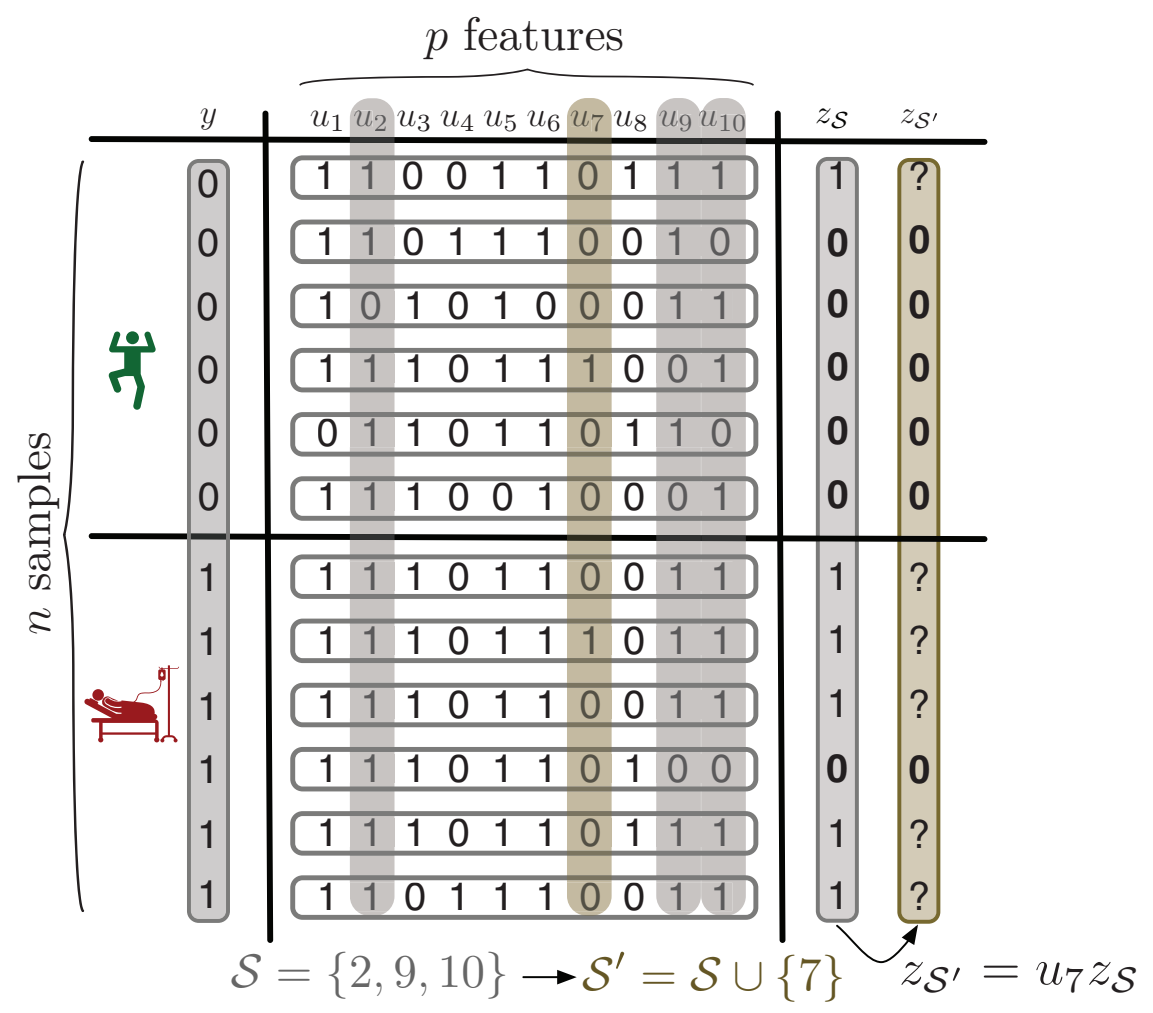

Figure 8.7: Illustration of how redundancy between patterns arises in significant itemset mining as a consequence of inclusion relationships $\mathcal{S}^{\prime} \subset \mathcal{S}$ between candidate patterns $\mathcal{S}, \mathcal{S}^{\prime} \in \mathcal{M}$.

Subset/superset relationships are not the only potential source of statistical redundancy between any two candidate patterns $\mathcal{S}_{1}, \mathcal{S}_{2} \in \mathcal{M}$. More generally, as long as the two patterns share some substructures, i.e., $\mathcal{S}_{1} \cap \mathcal{S}_{2} \neq \emptyset$, the random variables $G_{\mathcal{S}_{1}}(X)$ and $G_{\mathcal{S}_{2}}(X)$ might be statistically associated. The strength of this association and, therefore, the degree to which patterns $\mathcal{S}_{1}$ and $\mathcal{S}_{2}$ are statistically redundant, depends on the probability that the shared substructure $\mathcal{S}_{1} \cap \mathcal{S}_{2}$ occurs in an input sample $X$, relative to the probability that the pattern-specific substructures $\mathcal{S}_{1} \backslash \mathcal{S}_{2}$ and $\mathcal{S}_{2} \backslash \mathcal{S}_{1}$ occur. For example, if $\mathcal{S}_{1} \backslash \mathcal{S}_{2}$ and $\mathcal{S}_{2} \backslash \mathcal{S}_{1}$ are both common substructures that occur in almost every input sample $X$, the probability that $\mathcal{S}_{1}$ and $\mathcal{S}_{2}$ occur in an input sample $X$ will be dominated by the probability that $\mathcal{S}_{1} \cap \mathcal{S}_{2}$ occurs in $X$. Therefore, in this situation $\mathcal{S}_{1}$ and $\mathcal{S}_{2}$ will be considerably redundant. On the contrary, if $\mathcal{S}_{1} \cap \mathcal{S}_{2}$ is a common sub-structure, the probability that $\mathcal{S}_{1}$ occurs in an input sample $X$ will be mostly determined by how frequently $\mathcal{S}_{1} \backslash \mathcal{S}_{2}$ occurs, while the probability that $\mathcal{S}_{2}$ occurs in an input sample $X$ will mostly depend on how frequently $\mathcal{S}_{2} \backslash \mathcal{S}_{1}$ occurs. In this case, $G_{\mathcal{S}_{1}}(X)$ and $G_{\mathcal{S}_{2}}(X)$ will be approximately independent, leading to patterns $\mathcal{S}_{1}$ and $\mathcal{S}_{2}$ being barely redundant. 


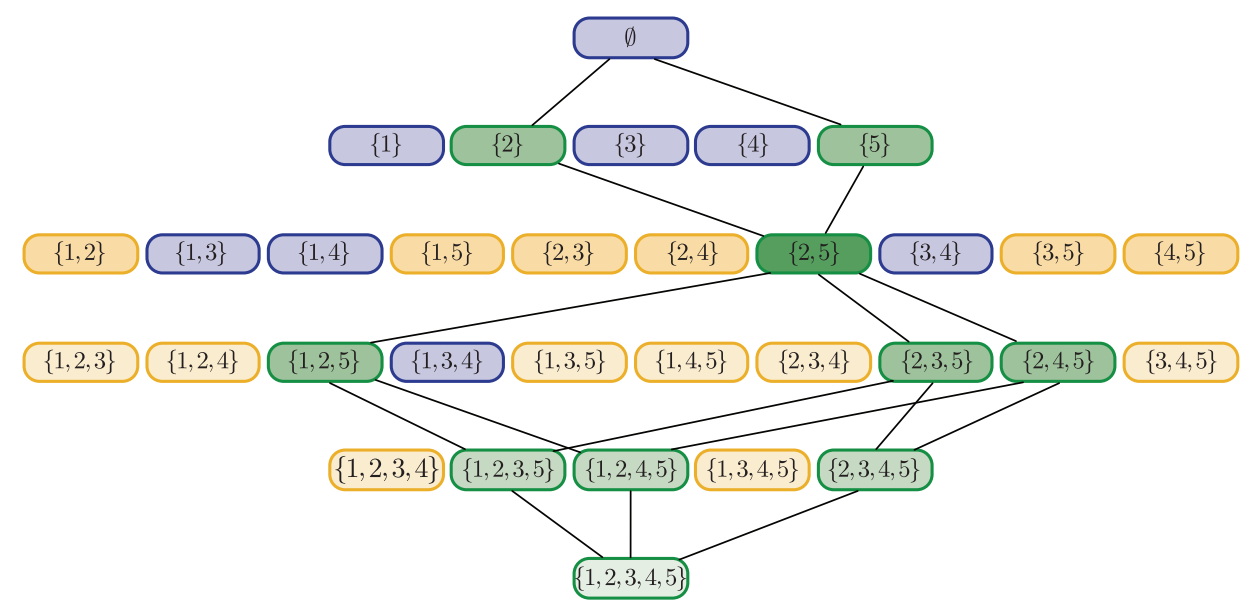

Figure 8.8: Illustration of how the sharing of pattern substructures and subset/superset relationships between patterns leads to any given pattern $\mathcal{S} \in \mathcal{M}$ being related to exponentially many other patterns in the search space $\mathcal{M}$. In this significant itemset mining example, a high-order feature interaction $\mathcal{S}=\{2,5\}$, highlighted in dark green, is related by subset/superset relationships to $9 / 32$ high-order feature interactions (green) and shares features with other 14/32 high-order feature interactions (orange). The intensity of a node's colour is proportional to the relatedness of the corresponding high-order feature interaction $\mathcal{S}^{\prime}$ with $\mathcal{S}=\{2,5\}$.

Due to the combinatorial nature of the search space $\mathcal{M}$, these statistical redundancies that arise due to the sharing of pattern substructures and subset/superset relationships can lead to any given pattern $\mathcal{S}$ being statistically associated with exponentially many other patterns $\mathcal{S}^{\prime}$. This effect is illustrated in Figure 8.8 for a significant itemset mining problem with $p=5$ features. In this example, a given feature interaction $\mathcal{S}=\{2,5\}$ (dark green) is directly related by subset/superset relationships to 9 out of 32 patterns (green) and shares some substructures (in this case, input features) with other 14 out of 32 patterns (orange). In short, in this example, pattern $\mathcal{S}=\{2,5\}$ is potentially redundant with more than half of the entire search space $\mathcal{M}$.

The existence of this complex web of inter-dependencies between patterns in the search space $\mathcal{M}$ has profound implications. If the random variables $G_{\mathcal{S}_{1}}(X)$ and $G_{\mathcal{S}_{2}}(X)$ are statistically dependent, the corresponding $P$-values $p_{\mathcal{S}_{1}}$ and $p_{\mathcal{S}_{2}}$ quantifying the statistical association of $G_{\mathcal{S}_{1}}(X)$ and $G_{\mathcal{S}_{2}}(X)$ with the class labels $Y$ might be statistically dependent as well. Suppose that patterns $\mathcal{S}_{1}, \mathcal{S}_{2} \in \mathcal{M}$ are not associated with the class labels $Y$, i.e., $\mathcal{S}_{1}, \mathcal{S}_{2} \in \mathcal{M}_{\text {null }}$ as defined in Section 8.2.3. Then, if $p_{\mathcal{S}_{1}}$ and $p_{\mathcal{S}_{2}}$ were strongly positively correlated, false positives for pattern $\mathcal{S}_{1}$, i.e., $\left[p_{\mathcal{S}_{1}} \leq \delta\right.$ ], will tend to co-occur with false positives for pattern $\mathcal{S}_{2}$, i.e., $\left[p_{\mathcal{S}_{2}} \leq \delta\right.$ ]. The implications of this observation can be traced back to the derivations of the FWER upper bounds used by the Bonferroni correction and Tarone's method. Both approaches make use of the basic fact that, as a consequence of the axioms of probability, $\operatorname{Pr}\left(\left[p_{\mathcal{S}_{1}} \leq\right.\right.$ $\left.\delta] \cup\left[p_{\mathcal{S}_{2}} \leq \delta\right]\right) \leq \operatorname{Pr}\left(p_{\mathcal{S}_{1}} \leq \delta\right)+\operatorname{Pr}\left(p_{\mathcal{S}_{2}} \leq \delta\right)$. However, if $p_{\mathcal{S}_{1}}$ and $p_{\mathcal{S}_{2}}$ had a strong positive correlation, $\operatorname{Pr}\left(\left[p_{\mathcal{S}_{1}} \leq \delta\right] \cup\left[p_{\mathcal{S}_{2}} \leq \delta\right]\right) \approx \operatorname{Pr}\left(p_{\mathcal{S}_{1}} \leq \delta\right) \approx \operatorname{Pr}\left(p_{\mathcal{S}_{2}} \leq \delta\right)$ will hold. More generally, if the set of $P$-values $\left\{p_{\mathcal{S}} \mid \mathcal{S} \in \mathcal{M}\right\}$ exhibits exponentially many 
statistical inter-dependencies, possible extending beyond simple pairwise associations between $P$-values, then $\operatorname{Pr}\left(\bigcup_{\mathcal{S} \in \mathcal{M}_{\text {null }}(\delta)}\left[p_{\mathcal{S}} \leq \delta\right]\right) \ll \sum_{\mathcal{S} \in \mathcal{M}_{\text {null }}(\delta)} \operatorname{Pr}\left(p_{\mathcal{S}} \leq \delta\right)$ and $\operatorname{Pr}\left(\bigcup_{\mathcal{S} \in \mathcal{M}_{\text {test }}(\delta)}\left[p_{\mathcal{S}} \leq \delta\right]\right) \ll \sum_{\mathcal{S} \in \mathcal{M}_{\text {test }}(\delta)} \operatorname{Pr}\left(p_{\mathcal{S}} \leq \delta\right)$. Hence, both the Bonferroni correction and Tarone's method tend to overestimate the real FWER.

In summary, by ignoring the existence of redundancies between patterns, statistical power will be lost as a consequence of overestimating the FWER. This opens the door to the development of novel approaches that are able to model these statistical dependencies between patterns, obtaining a more accurate approximation of the FWER and ultimately leading to a gain of statistical power.

The rest of this section is organized as follows. In Section 8.4.1, we present the Westfall-Young permutation-testing procedure as a way to empirically estimate the FWER under the global null hypothesis that no pattern is associated with the class labels. Next, in Section 8.4.2, we introduce an extension of the algorithm presented in Section 8.3 that incorporates permutation-testing to improve statistical power.

\subsubsection{Empirically Approximating the FWER Using Random Permutations}

Due to the difficulty to exactly evaluate the FWER at a given adjusted significance threshold $\delta$, both the Bonferroni correction and Tarone's method approximate $\operatorname{FWER}(\delta)=\operatorname{Pr}(\operatorname{FP}(\delta)>0)$ with an upper bound $\widehat{\operatorname{FWER}}(\delta)$. Tarone's method exploits the concept of testability to obtain an upper bound on the true FWER that is considerably more accurate, in significant pattern mining, than the Bonferroni correction. Nonetheless, both methods implicitly assume that all patterns $\mathcal{S} \in \mathcal{M}$ are statistically independent. As discussed above, this is rarely the case in significant pattern mining. As a consequence, Tarone's method still tends to overestimate the true FWER, leading to a potential loss of statistical power. An alternative approach to approximate $\operatorname{FWER}(\delta)$ is to use resampling techniques to obtain an empirical estimate $\widehat{\operatorname{FWER}}(\delta)$.

For this purpose, one of the most commonly used resampling schemes consists of applying random permutations to the class labels [47]. Let $\mathcal{D}=\left\{\left(x_{i}, y_{i}\right)\right\}_{i=1}^{n}$ be an input dataset with $n$ i.i.d. samples $x \in \mathcal{X}$ and class labels $y \in\{0,1\}$. Suppose that $\pi: \llbracket 1, n \rrbracket \rightarrow \llbracket 1, n \rrbracket$ is a random permutation, i.e. a permutation of the set $\llbracket 1, n \rrbracket$ selected uniformly at random from the set of all $n$ ! permutations of $\llbracket 1, n \rrbracket$. Define the resampled dataset $\widetilde{\mathcal{D}}=\left\{\left(x_{i}, y_{\pi(i)}\right)\right\}_{i=1}^{n}$ in such a way that the $i^{\text {th }}$ input sample $x_{i}$ is paired with the class label of sample $\pi(i)$, for each $i=1, \ldots, n$. The effect of obtaining the class labels in the resampled dataset $\widetilde{\mathcal{D}}$ by randomly permuting the original labels in $\mathcal{D}$ is to assign a random class label to each input sample while keeping the class ratio $n_{1} / n$ unchanged. As a consequence, any statistical dependency between patterns and labels which might have existed in the original dataset $\mathcal{D}$ is effectively eliminated by the permutation process. While the ground-truth regarding which patterns $\mathcal{S} \in \mathcal{M}$ are statistically associated with the class labels in the original dataset $\mathcal{D}$ is unknown, in the resampled dataset $\widetilde{\mathcal{D}}$, no pattern $\mathcal{S} \in \mathcal{M}$ can possibly be associated with the class labels. 


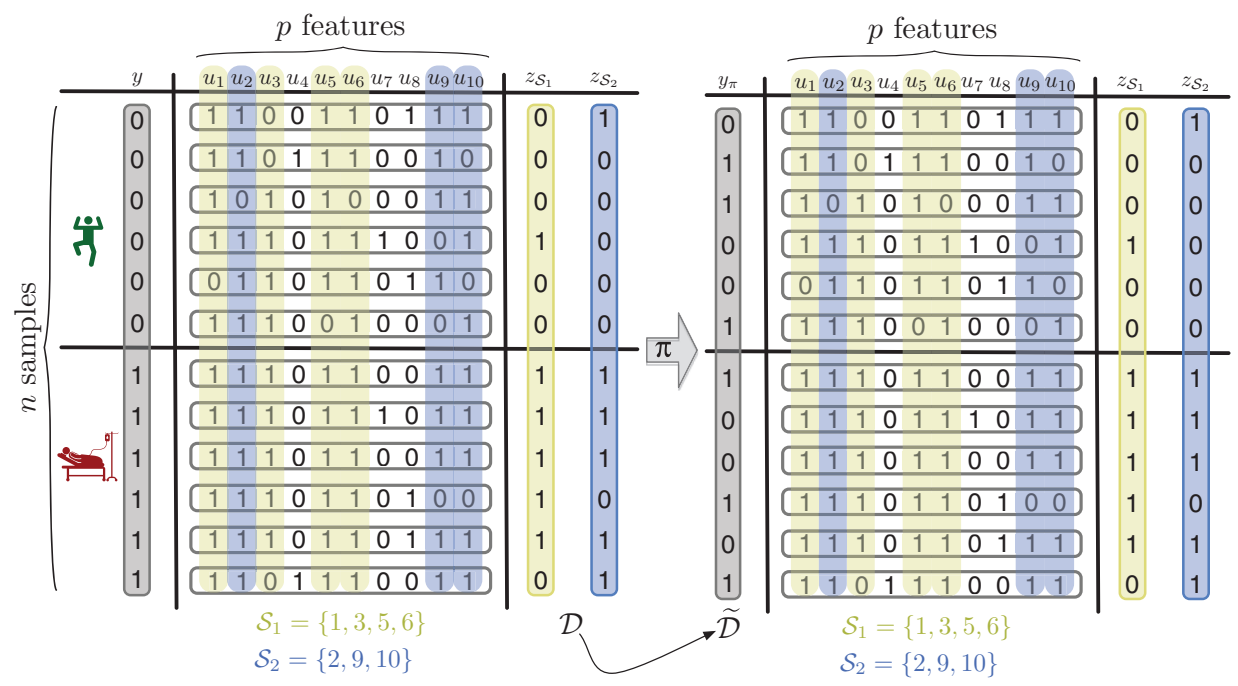

Figure 8.9: A random permutation of the class labels $\pi: \llbracket 1, n \rrbracket \rightarrow \llbracket 1, n \rrbracket$ can be used to derive a resampled dataset $\widetilde{\mathcal{D}}$ from an input dataset $\mathcal{D}$. In this significant itemset mining example, a dataset $\mathcal{D}$ with $n=12$ samples and $p=10$ features, shown on the left, has been resampled to obtain a new dataset $\widetilde{\mathcal{D}}$, shown on the right. While both datasets share the same samples $\left\{x_{i}\right\}_{i=1}^{n}$ and class ratio $n_{1} / n$, the mapping of class labels and samples is different. A consequence of this is that the occurrence of patterns $\mathcal{S}_{1}$ and $\mathcal{S}_{2}$, which is enriched in samples belonging to class $y=1$ in the original dataset $\mathcal{D}$, no longer show any association with the class labels in the resampled dataset $\widetilde{\mathcal{D}}$.

In other words, the global null hypothesis $\mathcal{M}_{\text {null }}=\mathcal{M}$ holds for $\widetilde{\mathcal{D}}$. An illustration of the permutation process is shown in Figure 8.9 for a significant itemset mining dataset.

The fact that a resampled dataset $\widetilde{\mathcal{D}}$ obtained in this manner is known to contain no associations can be exploited to obtain an empirical estimate of the FWER under the global null hypothesis that no pattern $\mathcal{S} \in \mathcal{M}$ is associated with the class labels. ${ }^{13}$ Suppose that the resampling process described above is repeated a number $j_{p}$ of times, leading to a set $\left\{\widetilde{\mathcal{D}}^{(k)}\right\}_{k=1}^{j_{p}}$ of $j_{p}$ resampled datasets. Leaving the matter of computational feasibility temporarily aside, suppose that for each dataset $\widetilde{\mathcal{D}}^{(k)}$, the $P$-values $p_{\mathcal{S}}^{(k)}$ for all patterns $\mathcal{S} \in \mathcal{M}$ were obtained in order to find the $P$-value corresponding to the most significant pattern, i.e. in order to compute $p_{\min }^{(k)}=\min \left\{p_{\mathcal{S}}^{(k)} \mid \mathcal{S} \in \mathcal{M}\right\}$. By construction, $p_{\text {min }}^{(k)} \leq p_{\mathcal{S}}^{(k)}$ for all patterns $\mathcal{S} \in \mathcal{M}$. Hence, if $p_{\min }^{(k)}>\delta$, no pattern $\mathcal{S} \in \mathcal{M}$ will be deemed significant in the $k^{\text {th }}$ resampled dataset $\widetilde{\mathcal{D}}^{(k)}$, leading to no false positives being detected for this resampled dataset $\left(\operatorname{FP}^{(k)}(\delta)=0\right)$. On the contrary, if

13 Controlling the FWER under the global null hypothesis is often denoted as weak control of the FWER. If the subset pivotality condition [47] can be shown to hold, it is possible to prove that permutation-testing also controls the FWER in the strong sense, i.e. when any subset of hypothesis is allowed to be non-null. However, permutation-testing has been extensively applied to problems for which the subset pivotality condition cannot be proven, as is the case of significant pattern mining, nevertheless leading to successful results. 
$p_{\text {min }}^{(k)} \leq \delta$, there is at least one pattern $\mathcal{S} \in \mathcal{M}$ being deemed significant for the $k$-th resampled dataset. Since $\widetilde{\mathcal{D}}^{(k)}$ is known to contain no associations, this implies that $\operatorname{FP}^{(k)}(\delta)>0$. Therefore, an empirical estimator of the FWER can be obtained as:

$$
\widehat{\operatorname{FWER}}(\delta)=\frac{1}{j_{p}} \sum_{k=1}^{j_{p}} \mathbb{1}\left[p_{\min }^{(k)} \leq \delta\right],
$$

where $\mathbb{1}[\bullet]$ evaluates to 1 if its input argument is true and to 0 otherwise. Intuitively, the estimator $\widehat{\operatorname{FWER}}(\delta)$ of the FWER at adjusted significance threshold $\delta$ is simply given by the proportion of the $j_{p}$ resampled datasets that contain at least one false positive. If the number of permutations $j_{p}$ is sufficiently large (e.g., $j_{p} \approx 10,000$ ), $\widehat{\operatorname{FWER}}(\delta)$ will be a rather accurate estimate of the true value $\operatorname{FWER}(\delta)$. An adjusted significance threshold can then be proposed based on this estimator as:

$$
\delta_{\text {perm }}=\max \left\{\delta \mid \frac{1}{j_{p}} \sum_{k=1}^{j_{p}} \mathbb{1}\left[p_{\min }^{(k)} \leq \delta\right] \leq \alpha\right\} .
$$

The empirical estimate of the FWER obtained via permutation testing implicitly accounts for the dependence structure that might exist between patterns. While this can lead to a considerable improvement in statistical power with respect to Tarone's method, a naive application of this procedure to significant pattern mining is entirely unfeasible. First, evaluating $p_{\text {min }}^{(k)}$ for a single resampled dataset $\widetilde{\mathcal{D}}^{(k)}$ is a challenging problem on its own. In principle, evaluating $p_{\min }^{(k)}$ naively would require computing the $P$-values $p_{\mathcal{S}}^{(k)}$ for all patterns $\mathcal{S} \in \mathcal{M}$, which is typically unfeasible due to the size of the search space $\mathcal{M}$. Moreover, in permutation testing, this operation needs to be repeated between $j_{p}=1,000$ and $j_{p}=10,000$ times if a sufficiently accurate estimate of the FWER is to be obtained.

In the next section, we will discuss how specific properties of the FWER estimator in Equation 8.26 can be exploited to allow the application of permutation-testing in significant pattern mining.

\subsubsection{Permutation Testing in Significant Pattern Mining}

The idea of using permutation testing to improve statistical power in significant pattern mining was pioneered by [18]. In their work, the authors explicitly tackle the problem of efficiently computing $p_{\text {min }}^{(k)}$ for a single resampled dataset $\widetilde{\mathcal{D}}^{(k)}$. In order to avoid computing the $P$-values $p_{\mathcal{S}}^{(k)}$ for all patterns $\mathcal{S} \in \mathcal{M}$ in the search space, their method relies again on the concept of minimum attainable $P$-value. Suppose that $\hat{p}_{\text {min }}^{(k)}$ is an estimate of $p_{\min }^{(k)}$ obtained after having enumerated only a subset of the search space $\mathcal{M}$. If the minimum attainable $P$-value $p_{\mathcal{S} \text {, min }}$ of a pattern $\mathcal{S}$ satisfies $p_{\mathcal{S}, \text { min }}>\hat{p}_{\text {min }}^{(k)}$, then $\min \left(\hat{p}_{\min }^{(k)} p_{\mathcal{S}}^{(k)}\right)=\hat{p}_{\min }^{(k)}$. In other words, if the minimum attainable $P$-value $p_{\mathcal{S} \text {, min }}$ of a pattern $\mathcal{S}$ is larger than the smallest $P$-value $\hat{p}_{\min }^{(k)}$ among all patterns enumerated so far, pattern $\mathcal{S}$ has no chance of having a $P$-value smaller than $\hat{p}_{\text {min }}^{(k)}$. Hence, $p_{\mathcal{S}}^{(k)}$ does not need to be computed. Most importantly, this idea can be combined 


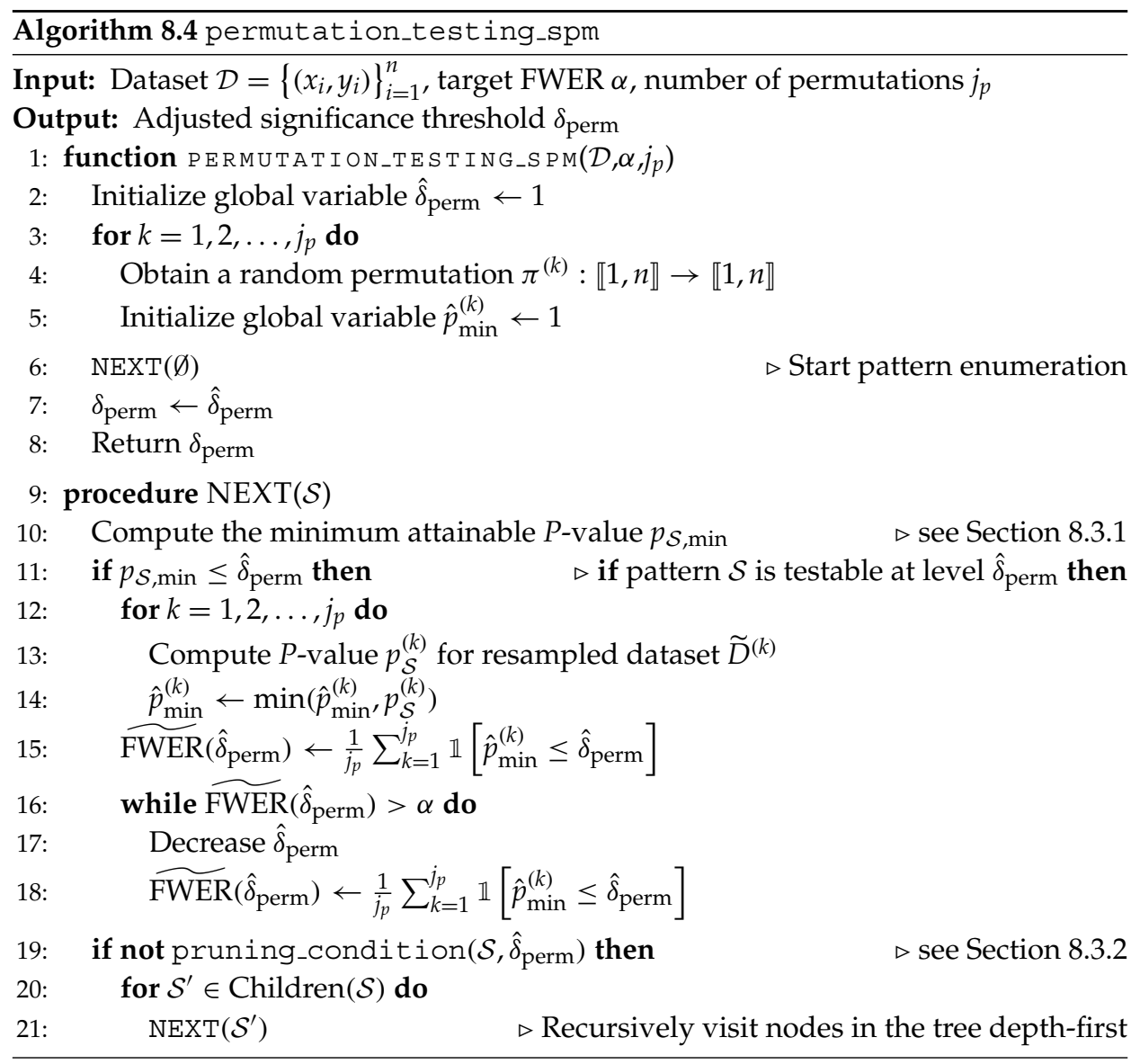

with the powerful concept of search space pruning described in Section 8.3: If $p_{\mathcal{S}, \min }>$ $\hat{p}_{\min }^{(k)}$ and $r_{\mathcal{S}} \leq \min \left(n_{1}, n-n_{1}\right)$, no descendant $\mathcal{S}^{\prime} \in \operatorname{Children}(\mathcal{S})$ of $\mathcal{S}$ in the enumeration tree can possibly satisfy $p_{\mathcal{S}^{\prime}}^{(k)} \leq \hat{p}_{\min }^{(k)}$, naturally leading to a valid search space pruning criterion analogous to the one employed by Algorithm 8.2.

While the algorithm proposed in [18] is able to obtain the exact value of $p_{\min }^{(k)}$ while enumerating only a small subset of patterns in the search space $\mathcal{M}$, it is still limited by the fact that this operation needs to be repeated around $j_{p} \approx 10,000$ times. In particular, the whole pattern enumeration process has to be repeated independently for each permutation, leading to a computational overhead that limits the applicability of the method to datasets of small-to-moderate size. Building upon the work in [18], an alternative approach to apply permutation testing in significant pattern mining was proposed in [19]. Unlike the previous method, this approach processes all resampled datasets simultaneously, requiring to enumerate patterns only a single time. In practice, this leads to a drastic reduction in runtime and memory usage that allows scaling-up the method to considerably larger datasets.

Pseudocode describing the approach in [19] is shown in Algorithm 8.4. The skeleton of the method closely parallels Algorithm 8.2. The search space $\mathcal{M}$ of all candidate 
patterns is explored in the same way: Recursively traversing a pattern enumeration tree that satisfies $\mathcal{S}^{\prime} \in \operatorname{Children}(\mathcal{S}) \Rightarrow \mathcal{S} \subseteq \mathcal{S}^{\prime}$ depth-first. The algorithm begins by initializing the estimate $\hat{\delta}_{\text {perm }}$ of the adjusted significance threshold to 1 (Line 2 ). Next, in Lines 3-5, for each of the $j_{p}$ resampled datasets, the algorithm precomputes the random permutation of the class labels and initializes the estimate $\hat{p}_{\min }^{(k)}$ of the most significant $P$-value to 1 . After the initialization phase, the algorithm proceeds to start the pattern enumeration procedure at the root of the tree (Line 6). For each pattern $\mathcal{S}$ visited during the traversal of the enumeration tree, Algorithm 8.4 first computes the minimum attainable $P$-value $p_{\mathcal{S} \text {, } \min }$ in Line 10 . The algorithm then proceeds differently depending on the testability of pattern $\mathcal{S}$.

If pattern $\mathcal{S}$ is testable at level $\hat{\delta}_{\text {perm }}$ then, for each of the $j_{p}$ resampled datasets $\widetilde{D}^{(k)}$, the algorithm computes the $P$-value $p_{\mathcal{S}}^{(k)}$ and updates the estimate $\hat{p}_{\text {min }}^{(k)}$ of the most significant $P$-value for the $k$-th resampled dataset (Lines 12-14). Next, in Line 15, an estimate $\widehat{\text { FWER }}\left(\hat{\delta}_{\text {perm }}\right)$ of the FWER at level $\hat{\delta}_{\text {perm }}$ is obtained using the estimates $\hat{p}_{\text {min }}^{(k)}$ of the most significant $P$-value for each resampled dataset. Since the enumeration process is not yet completed, $\hat{p}_{\min }^{(k)} \geq p_{\min }^{(k)}$ leading to $\frac{1}{j_{p}} \sum_{k=1}^{j_{p}} \mathbb{1}\left[\hat{p}_{\min }^{(k)} \leq \hat{\delta}_{\text {perm }}\right]=$ $\widetilde{\operatorname{FWER}}\left(\hat{\delta}_{\text {perm }}\right)$ being a lower bound of $\widehat{\operatorname{FWER}}\left(\hat{\delta}_{\text {perm }}\right)=\frac{1}{j_{p}} \sum_{k=1}^{j_{p}} \mathbb{1}\left[p_{\min }^{(k)} \leq \hat{\delta}_{\text {perm }}\right]$. Thus, if we have $\widetilde{F W E R}\left(\hat{\delta}_{\text {perm }}\right) \geq \alpha$, then $\widehat{\operatorname{FWER}}\left(\hat{\delta}_{\text {perm }}\right) \geq \alpha$ as well, implying that the FWER condition is violated. In Lines 16-18, Algorithm 8.4 checks this condition and, if found to be violated, decreases he estimate $\hat{\delta}_{\text {perm }}$ of the adjusted significance threshold until the FWER condition is satisfied again.

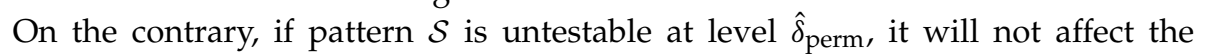
values of the FWER estimator $\widetilde{\operatorname{FWER}}(\delta)=\frac{1}{j_{p}} \sum_{k=1}^{j_{p}} \mathbb{1}\left[\hat{p}_{\text {min }}^{(k)} \leq \delta\right]$ for any $\delta \leq \hat{\delta}_{\text {perm }}$. Consequently, the computation of the $P$-values $p_{\mathcal{S}}^{(k)}$ for $k=1, \ldots, j_{p}$ can be skipped, as well as the update of the FWER estimator FWER $\left(\hat{\delta}_{\text {perm }}\right)$. The fact that untestable patterns cannot modify the value of $\widetilde{\operatorname{FWER}}(\delta)$ follows from the definition of testability.

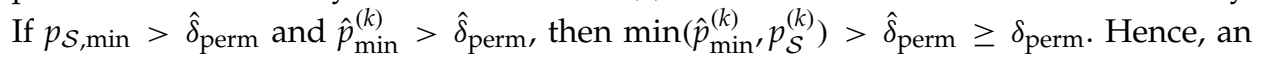
untestable pattern $\mathcal{S}$ is irrelevant as far as the FWER is concerned, also for permutation testing.

Search space pruning is fundamental to the computational feasibility of Algorithm 8.4, as is for other significant pattern mining algorithms. As shown in Section 8.3.2, when Pearson's $\chi^{2}$ test or Fisher's exact test are used, descendants $\mathcal{S}^{\prime}$ of an untestable pattern $\mathcal{S}$ will also be untestable provided that $r_{\mathcal{S}} \leq \min \left(n_{1}, n-n_{1}\right)$. Reiterating the argument above, this implies that those descendants cannot affect the value of $\widetilde{\mathrm{FWER}}(\delta)$ and, thus, can be pruned from the search space. In other words, the pruning condition of Algorithm 8.4 is identical to that of Algorithm 8.2. Finally, Lines 20-21 continue the traversal of the tree recursively, visiting the children of patterns for which the pruning condition does not apply.

As was the case for Algorithm 8.2, $\hat{\delta}_{\text {perm }}$ progressively decreases as patterns are enumerated, leading to less patterns being testable and the pruning condition becoming more stringent. Eventually, the algorithm ends after all patterns have either been pruned or visited. Due to the way the algorithm proceeds, once that occurs, $\hat{\delta}_{\text {perm }}=$ $\delta_{\text {perm, }}$ allowing the value to be returned in Line 8 . Once the adjusted significance 
threshold $\delta_{\text {perm }}$ has been obtained, Algorithm 8.3 can be used to retrieve all patterns $\mathcal{S}$ that are statistically significant at level $\delta_{\text {perm }}$.

Compared to Algorithm 8.2, the approach proposed by [19] and described in Algorithm 8.4 will exhibit more statistical power, due the use of permutation-testing to obtain a better approximation to the FWER. However, it is a more computationally demanding approach, as $j_{p} P$-values need to be computed for each pattern deemed testable during the pattern enumeration procedure. In short, Algorithm 8.2 allows to trade-off computational complexity for statistical power, an option that might be desirable in applications where signals are too weak to be detected by Algorithm 8.2.

\subsection{Accounting for a Categorical Covariate}

The need to incorporate into the model covariate factors that might have a confounding effect is an ubiquitous problem in computational biology and clinical data analysis. By neglecting to account for such covariates, an algorithm might find many spurious patterns whose association with the class labels is entirely mediated by confounding.

Let $G_{\mathcal{S}}(X)$ denote the binary random variable indicating the occurrence of pattern $\mathcal{S}$ in an input sample $X, Y$ the binary class label and $C$ be a random variable corresponding to a covariate factor taking values in a domain $\mathcal{C}$. Mathematically, the aforementioned situation occurs when:

1. $G_{\mathcal{S}}(X)$ and $Y$ are marginally statistically associated, i.e., $G_{\mathcal{S}}(X) \not \Perp \Perp N Y$. As discussed in Section 8.2.2, this is the case if and only if $\exists(x, y) \in\{0,1\}^{2}$ such that $\operatorname{Pr}\left(G_{\mathcal{S}}(X)=g_{\mathcal{S}}(x), Y=y\right) \neq \operatorname{Pr}\left(G_{\mathcal{S}}(X)=g_{\mathcal{S}}(x)\right) \operatorname{Pr}(Y=y)$.

2. $G_{\mathcal{S}}(X)$ and $Y$ are conditionally independent given $C$, i.e. $G_{\mathcal{S}}(X) \not 1 Y \mid C$. This occurs if and only if $\operatorname{Pr}\left(G_{\mathcal{S}}(X)=g_{\mathcal{S}}(x), Y=y \mid C=c\right)=\operatorname{Pr}\left(G_{\mathcal{S}}(X)=g_{\mathcal{S}}(x) \mid\right.$ $C=c) \operatorname{Pr}(Y=y \mid C=c) \forall c \in \mathcal{C}$.

Intuitively, condition (1) above implies that $G_{\mathcal{S}}(X)$ and $Y$ appear to be statistically associated in the absence of information about $C$, while condition (2) implies that, once the value $c$ taken by the covariate $C$ is known, $G_{\mathcal{S}}(X)$ carries no further information about $Y$ and, therefore, can be discarded. In most applications, patterns $\mathcal{S} \in \mathcal{M}$ satisfying conditions (1) and (2) are spurious findings that should not be retrieved by a mining algorithm.

These patterns will be of little use to the practitioner, as they provide no additional information about the class membership of an input sample beyond the information that is already contained in the covariate. In many applications, the covariates are quantities that can be measured more easily than the input samples $x \in \mathcal{X}$. For example, while $x$ might represent a set of gene expression profiles or be a representation of a patient's genotype based on a set of single nucleotide polymorphisms, the covariate $C$ often represents simple information such as age, gender, or genetic ancestry. Therefore, from a practical point of view, if a marginally associated pattern $\mathcal{S} \in \mathcal{M}$ is redundant with such a covariate, it might be preferable to simply make use of the covariate when trying to predict the class label $Y$. 
Moreover, not only are patterns satisfying conditions (1) and (2) of little practical use but, in many occasions, they might represent misleading associations. A particularly common example are spurious associations between genotype and phenotype which arise in genome-wide association studies due to population structure [48]. Often, a phenotype might be strongly associated with the genetic ancestry of an individual, which obviously is itself associated with an individual's genotype. Therefore, if population structure is unaccounted for in a genome-wide association study containing individuals with diverse genetic ancestries, a large number of apparently significant patterns might be retrieved. However, a practitioner might later find that, in fact, most of these patterns simply correspond to genotypic motifs that differ among individuals with different genetic ancestries and contain no additional information about the phenotype.

The effect of confounding is illustrated in Figure 8.10. In this example, the same significant itemset mining dataset depicted in Figure 8.1 is shown. Patterns $\mathcal{S}_{1}$ and $\mathcal{S}_{2}$ are both marginally associated with the class labels, being clearly enriched among samples of class $y=1$. However, Figure 8.10 incorporates a factor not present in the original example: a categorical covariate $C$ with $k=2$ categories, representing the genetic ancestry of a sample. The inclusion of $C$ dramatically changes the interpretation of the associations that patterns $\mathcal{S}_{1}$ and $\mathcal{S}_{2}$ represent. While the occurrence of pattern $\mathcal{S}_{1}$ still carries additional information not present in the covariate, pattern $\mathcal{S}_{2}$ can be seen to be entirely redundant with the covariate $C$. Therefore, following our discussion above, pattern $\mathcal{S}_{2}$ might be considered a spurious association that should ideally not be retrieved.

Most significant pattern mining algorithms, including Algorithm 8.2 in Section 8.3 and Algorithm 8.4 in Section 8.4, are unable to account for covariates. Hence, they are prone to discover many spurious patterns due to confounding, limiting their applicability in computational biology and clinical data analysis. In order to solve this problem, a significant pattern mining approach able to account for the effect of covariates needs to be used. All methods that have been discussed in this chapter so far aim at finding all patterns $\mathcal{S} \in \mathcal{M}$ that are (marginally) statistically associated with the class labels, i.e., look for the set of patterns $\left\{\mathcal{S} \in \mathcal{M} \mid G_{\mathcal{S}}(X) \not \mathbb{L} Y\right\}$. However, an approach able to correct for the effect of a covariate $C$ would aim to find the set of patterns $\left\{\mathcal{S} \in \mathcal{M}\left|G_{\mathcal{S}}(X) \not \mathbb{} Y\right| C\right\}$ instead. In particular, a pattern $\mathcal{S} \in \mathcal{M}$ satisfying conditions (1) and (2) above would not be retrieved by the latter formulation yet it would be deemed significant by the former.

Recently, a novel significant pattern mining algorithm able to account for the effect of a categorical covariate $C$ has been proposed [20]. In a follow-up study [49], the techniques proposed in [20] have been used to develop a method able to aggregate weak effects in genome-wide association studies while correcting for confounding effects due to population structure. Unlike existing approaches, such as burden tests [50], the approach in [49] uses significant pattern mining to test all genomic regions, regardless of starting position and size. Empirically, this leads to a gain of statistical power over burden tests, whose statistical performance is shown to be sensitive to misspecification of the size of the genomic regions to be tested.

The approach in [20] follows closely Algorithm 8.2, described in Section 8.3. However, in order to incorporate the covariate $C$ into the model, it replaces Pearson's $\chi^{2}$ 


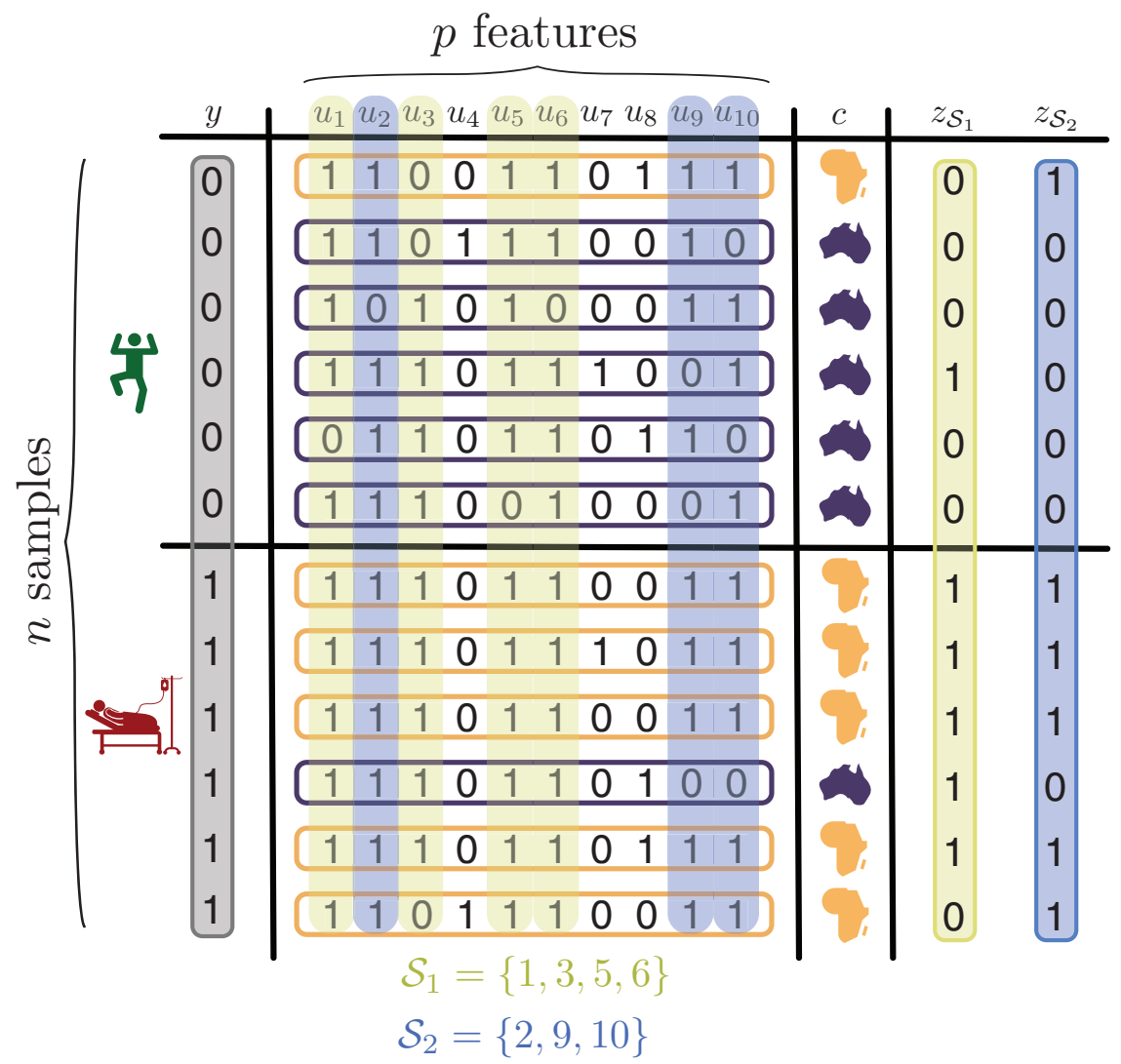

Figure 8.10: A simple illustration of the effect of confounding in a significant itemset mining dataset. A categorical covariate $C$ with $k=2$ categories (orange and purple) has been included. Two patterns, $\mathcal{S}_{1}$ and $\mathcal{S}_{2}$, are marginally associated with the class labels $Y$. However, only pattern $\mathcal{S}_{1}$ remains associated with $Y$ given the covariate $C$. On the contrary, the high-order feature interaction indexed by pattern $\mathcal{S}_{2}$ is active if and only if the sample is of African ancestry (orange). Thus, pattern $\mathcal{S}_{2}$ carries no information about $Y$ given that the value of $C$ is known, i.e., $\mathcal{S}_{2}$ is conditionally independent of $Y$ given $C$.

test or Fisher's exact test by the Cochran-Mantel-Haenszel (CMH) test [51]. Unlike the former two, the $\mathrm{CMH}$ test allows asessing the conditional association of two binary random variables $G_{\mathcal{S}}(X)$ and $Y$ given a categorical random variable $C$ with $k$ categories, making it ideal for this task. However, replacing the test statistic has implications for Tarone's method and its integration into the pattern mining algorithm. In particular, it is necessary to:

1. Prove that a minimum attainable $P$-value exists for the $\mathrm{CMH}$ test and derive an efficient expression to compute it.

2. Propose a novel search space pruning criterion that applies to the $\mathrm{CMH}$ test. 
The remainder of this section is organized as follows. In Section 8.5.1, we introduce the Cochran-Mantel-Haenszel (CMH) test in detail. Next, in Section 8.5.2, we discuss the derivation of a minimum attainable $P$-value for the $\mathrm{CMH}$ test. Finally, Section 8.5.3 introduces a valid pruning condition for the $\mathrm{CMH}$ test.

\subsubsection{Conditional Association Testing in Significant Pattern Mining}

In Section 8.2.2, $2 \times 2$ contingency tables were introduced as a way to represent the joint distribution of two binary random variables or its empirical approximation based on counts derived from $n$ i.i.d. samples. Based on these counts, two test statistics to assess the statistical association between two binary random variables were proposed: Pearson's $\chi^{2}$ test and Fisher's exact test. These were proposed in order to test the (marginal) association between the binary random variable $G_{\mathcal{S}}(X)$ representing the occurrence of a pattern $\mathcal{S} \in \mathcal{M}$ in an input sample $X$ and the class labels $Y$, based on an input dataset $\mathcal{D}=\left\{\left(x_{i}, y_{i}\right)\right\}_{i=1}^{n}$.

In this section, however, we are given a dataset $\mathcal{D}=\left\{\left(x_{i}, y_{i}, c_{i}\right)\right\}_{i=1}^{n}$ with $n$ i.i.d. samples $x \in \mathcal{X}$ belonging to one of two classes $y \in\{0,1\}$. Additionally, now each of the $n$ samples is also tagged with a categorical covariate $c \in\{1,2, \ldots, k\}$, where $k$ is the number of distinct categories that random variable $C$ can take. Unlike in the previous scenario, the new goal is to test the conditional association between $G_{\mathcal{S}}(X)$ and $Y$ given $C$. This is precisely what the Cochran-Mantel-Haenszel $(\mathrm{CMH})$ test was designed for.

Intuitively, the $\mathrm{CMH}$ test can be seen as a way to tackle this problem by reducing it to a set of $k$ instances of Pearson's $\chi^{2}$ test and then combining the $k$ resulting statistics appropriately. By definition, $G_{\mathcal{S}}(X)$ and $Y$ are conditionally independent given $C$ and, therefore, not conditionally associated given $C$, if $\operatorname{Pr}\left(G_{\mathcal{S}}(X)=g_{\mathcal{S}}(x), Y=y \mid C=\right.$ $c)=\operatorname{Pr}\left(G_{\mathcal{S}}(X)=g_{\mathcal{S}}(x) \mid C=c\right) \operatorname{Pr}(Y=y \mid C=c) \forall c \in\{1,2, \ldots, k\}$. For each $c=1, \ldots, k$, define $\mathcal{D}(c)=\left\{\left(x_{i}, y_{i}\right) \in \mathcal{D} \mid c_{i}=c\right\}$ to be the set of input samples in $\mathcal{D}$ for which the categorical covariate takes value $c$. The (unknown) joint distribution $\operatorname{Pr}\left(G_{\mathcal{S}}(X)=g_{\mathcal{S}}(x), Y=y \mid C=c\right)$ can be empirically approximated using counts derived from $\mathcal{D}(c)$ :

\begin{tabular}{|c|c|c|c|}
\hline Variables & $g_{\mathcal{S}}(x)=1$ & $g_{\mathcal{S}}(x)=0$ & Row totals \\
\hline$y=1$ & $a_{\mathcal{S}, c}$ & $b_{\mathcal{S}, c}$ & $n_{1, c}$ \\
\hline$y=0$ & $d_{\mathcal{S}, c}$ & $c_{\mathcal{S}, c}$ & $n_{0, c}$ \\
\hline Col. totals & $r_{\mathcal{S}, c}$ & $q_{\mathcal{S}, c}$ & $n_{c}$ \\
\hline
\end{tabular}

The interpretation of these counts is similar to the unconditional case described in Section 8.2.2. For instance, $a_{\mathcal{S}, c}$ is the number of samples in $\mathcal{D}(c)$ belonging to class $y=$ 1 for which pattern $\mathcal{S}$ occurs or, equivalently, the number of samples in $\mathcal{D}$ belonging to class $y=1$ for which pattern $\mathcal{S}$ occurs and the covariate takes value $c$. Consequently, an empirical approximation to $\operatorname{Pr}\left(G_{\mathcal{S}}(X)=1, Y=1 \mid C=c\right.$ ) could be obtained as $a_{\mathcal{S}, c} / n_{c}$ for each $c \in\{1,2, \ldots, k\}$. The remaining counts can be described analogously.

As a consequence of Proposition 8.1 in Section 8.2.2, if $G_{\mathcal{S}}(X)$ is conditionally independent of $Y$ given $C$, the random variable $A_{\mathcal{S}, c}$ given margins $n_{1, c}$ and $r_{\mathcal{S}, c}$ and sample size $n_{\mathcal{c}}$ follows a hypergeometric distribution with parameters $n_{c}, n_{1, c}$ and $r_{\mathcal{S}, c}$ 
for all $c \in\{1,2, \ldots, k\}$. Besides, under the assumption that all $n$ samples in $\mathcal{D}$ are obtained as i.i.d. draws, it follows that $A_{\mathcal{S}, c}$ is statistically independent of $A_{\mathcal{S}, c^{\prime}}$ for any $c \neq c^{\prime}$, since $\mathcal{D}(c) \cap \mathcal{D}\left(c^{\prime}\right)=\emptyset$. Paralleling the derivation of Pearson's $\chi^{2}$ test described in Section 8.2.2, the following $Z$-score can be proposed as a way to additively aggregate the individual $Z$-scores of the $k 2 \times 2$ contingency tables:

$$
Z_{\mathrm{cmh}}\left(\mathbf{a}_{\mathcal{S}} \mid \mathbf{n}, \mathbf{n}_{\mathbf{1}}, \mathbf{r}_{\mathcal{S}}\right)=\frac{\sum_{c=1}^{k} a_{\mathcal{S}, c}-\mathbb{E}\left[a_{\mathcal{S}, c} \mid R_{\mathcal{S}, c}=r_{\mathcal{S}, c}, N_{1, c}=n_{1, c}, H_{0}\right]}{\sqrt{\sum_{c=1}^{k} \operatorname{Var}\left[a_{\mathcal{S}, c} \mid R_{\mathcal{S}, c}=r_{\mathcal{S}, c}, N_{1, c}=n_{1, c}, H_{0}\right]}}
$$

where the term in the denominator follows from the fact that the variance of a sum of independent random variables equals the sum of the variances of each random variable participating in the sum. To simplify the notation, we introduced the vectors $\mathbf{a}_{\mathcal{S}}=\left(a_{\mathcal{S}, 1}, \ldots, a_{\mathcal{S}, k}\right), \mathbf{n}=\left(n_{1}, \ldots, n_{k}\right), \mathbf{n}_{\mathbf{1}}=\left(n_{1,1}, \ldots, n_{1, k}\right)$ and $\mathbf{r}_{\mathcal{S}}=\left(r_{\mathcal{S}, 1}, \ldots, r_{\mathcal{S}, k}\right)$, which contain the values of $a_{\mathcal{S}, c}, n_{c}, n_{1, c}$ and $r_{\mathcal{S}, c}$ for all $k 2 \times 2$ contingency tables. The final expression for the $\mathrm{CMH}$ test can be obtained by squaring this Z-score and plugging in the values of $\mathbb{E}\left[a_{\mathcal{S}, c} \mid R_{\mathcal{S}, c}=r_{\mathcal{S}, c}, N_{1, c}=n_{1, c}, H_{0}\right]$ and $\operatorname{Var}\left[a_{\mathcal{S}, c} \mid R_{\mathcal{S}, c}=\right.$ $\left.r_{\mathcal{S}, c}, N_{1, c}=n_{1, c}, H_{0}\right]$ as the mean and variance of a hypergeometric distribution with parameters $n_{c}, n_{1, c}$ and $r_{\mathcal{S}, c}$ :

$$
T_{\mathrm{cmh}}\left(\mathbf{a}_{\mathcal{S}} \mid \mathbf{n}, \mathbf{n}_{\mathbf{1}}, \mathbf{r}_{\mathcal{S}}\right)=\frac{\left(\sum_{c=1}^{k} a_{\mathcal{S}, c}-r_{\mathcal{S}, c} \frac{n_{1, c}}{n_{c}}\right)^{2}}{\sum_{c=1}^{k} \frac{r_{\mathcal{S}, c}}{n_{c}} \frac{n_{c}-r_{\mathcal{S}, c}}{n_{c}} \frac{n_{c}-n_{1, c}}{n_{c}-1} n_{1, c}}
$$

$T_{\mathrm{cmh}}\left(\mathbf{a}_{\mathcal{S}} \mid \mathbf{n}, \mathbf{n}_{1}, \mathbf{r}_{\mathcal{S}}\right)$ therefore aggregates evidence against the null hypothesis $H_{0}$ that $G_{\mathcal{S}}(X)$ is conditionally independent of $Y$ given $C$ across all $k 2 \times 2$ contingency tables. Large values of the test statistic are less likely to occur if the null hypothesis $H_{0}$ holds.

An approximation to the null distribution of the $\mathrm{CMH}$ test statistic can be obtained using the same arguments employed to derive an approximation to the null distribution of Pearson's $\chi^{2}$ test statistic. For a sufficiently large sample size $n$, the null distribution of $Z_{\mathrm{cmh}}\left(\mathbf{a}_{\mathcal{S}} \mid \mathbf{n}, \mathbf{n}_{\mathbf{1}}, \mathbf{r}_{\mathcal{S}}\right)$ will be approximately a standard Gaussian. Thus, the null distribution of $T_{\mathrm{cmh}}\left(\mathbf{a}_{\mathcal{S}} \mid \mathbf{n}, \mathbf{n}_{1}, \mathbf{r}_{\mathcal{S}}\right)$ can be approximated as a $\chi_{1}^{2}$ distribution and the corresponding two-tailed $P$-value can be obtained as:

$$
p_{\mathrm{cmh}}\left(\mathbf{a}_{\mathcal{S}} \mid \mathbf{n}, \mathbf{n}_{\mathbf{1}}, \mathbf{r}_{\mathcal{S}}\right)=1-F_{\chi_{1}^{2}}\left(T_{\mathrm{cmh}}\left(\mathbf{a}_{\mathcal{S}} \mid \mathbf{n}, \mathbf{n}_{\mathbf{1}}, \mathbf{r}_{\mathcal{S}}\right)\right),
$$

where $F_{\chi_{1}^{2}}(\bullet)$ is the cumulative density function of a $\chi_{1}^{2}$ distribution.

In Figure 8.11, we illustrate the result of applying the $\mathrm{CMH}$ test to assess the statistical significance of pattern $\mathcal{S}_{2}$ in the dataset previously shown in Figure 8.10. The $2 \times 2$ contingency table built using all samples in the dataset $\mathcal{D}$, shown at the top of the figure (light blue), clearly suggests a (marginal) association between $G_{\mathcal{S}_{2}}(X)$ and $Y$. Indeed, if Pearson's $\chi^{2}$ test is used to compute a $P$-value, one obtains $p_{\text {Pearson }}\left(a_{\mathcal{S}_{2}} \mid\right.$ $\left.n, n_{1}, r_{\mathcal{S}_{2}}\right)=0.021$, a rather significant result taking into account that the sample size is only $n=12$. However, if this contingency table is split into $k=2$ distinct tables according to the value of the categorical covariate, leading to the orange and purple contingency tables shown at the bottom of the figure, this association can be seen to disappear. In particular, those tables are so extreme that only one outcome for $a_{\mathcal{S}_{2, c}}$ is possible in each case, i.e., $a_{\mathcal{S}_{2}, c, \min }=a_{\mathcal{S}_{2}, c, \max }$ holds for both. Consequently, the CMH test leads to an entirely non-significant $P$-value $p_{\mathrm{cmh}}\left(\mathbf{a}_{\mathcal{S}_{2}} \mid \mathbf{n}, \mathbf{n}_{1}, \mathbf{r}_{\mathcal{S}_{2}}\right)=1$, successfully 


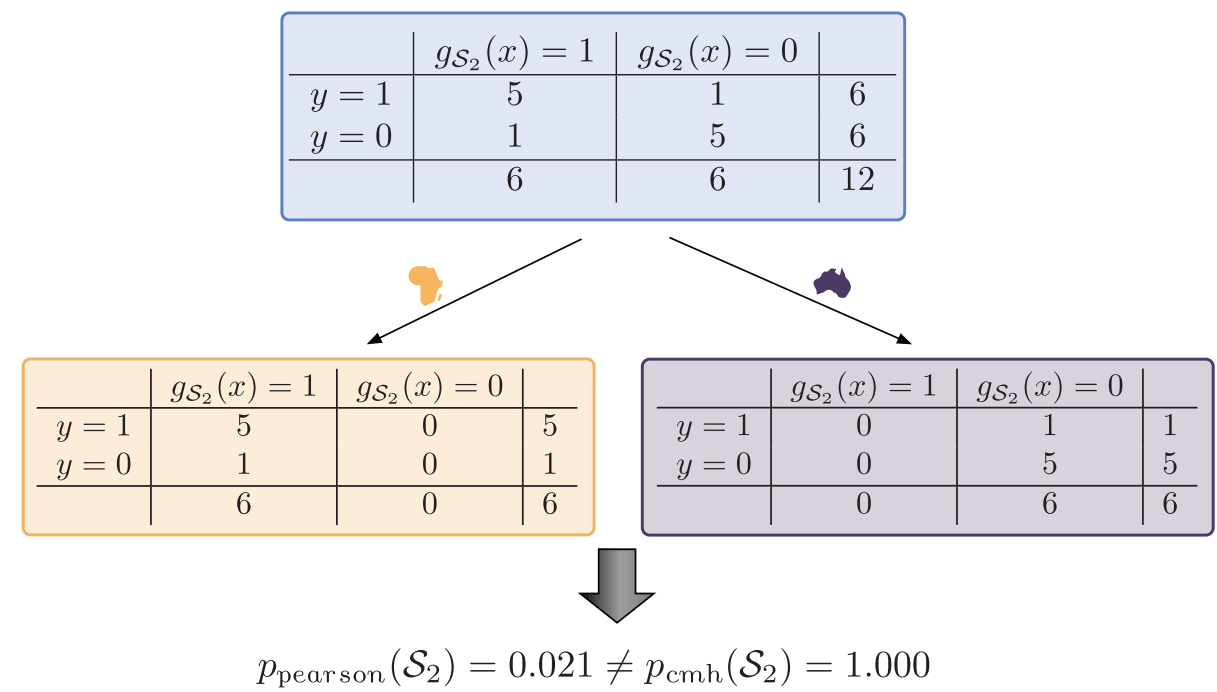

Figure 8.11: Result of using the $\mathrm{CMH}$ test to quantify the statistical association between class labels $Y$ and the occurrence of pattern $\mathcal{S}_{2}$ in the input samples, given the categorical covariate $C$. The light blue contingency table shown at the top of the figure is built using all samples in the dataset $\mathcal{D}$, while the two contingency tables shown at the bottom of the figure are obtained from the stratified datasets $\mathcal{D}(1)$ and $\mathcal{D}(2)$. In this case, category $c=1$ has been associated with samples of African ancestry (orange) and category $c=2$ with samples of Australian ancestry (purple).

correcting the confounding effect of the covariate. In contrast, it can be readily verified that if this analysis is repeated for pattern $\mathcal{S}_{1}$, which is not affected by confounding in the example of Figure 8.10, the $\mathrm{CMH}$ test still returns a rather significant $P$-value, $p_{\mathrm{cmh}}\left(\mathbf{a}_{\mathcal{S}_{1}} \mid \mathbf{n}, \mathbf{n}_{1}, \mathbf{r}_{\mathcal{S}_{1}}\right)=0.029$.

\subsubsection{Deriving the Minimum Attainable $P$-value for the CMH Test}

As Pearson's $\chi^{2}$ test and Fisher's exact test, the CMH test is based on discrete data and, as a consequence, there is a finite number of distinct values it can attain. As discussed in Section 8.2.3, this property is essential for the application of Tarone's method, as it makes it possible to obtain a minimum attainable $P$-value strictly larger than zero. This leads to the following proposition:

Proposition 8.6 (Minimum attainable $P$-value for the Cochran-Mantel-Haenszel $(\mathrm{CMH})$ test $)$ Define $\mathbf{a}_{\mathcal{S}, \min }=\left(a_{\mathcal{S}, 1, \min }, a_{\mathcal{S}, 2, \min } \ldots, a_{\mathcal{S}, k, \min }\right)$ and $\mathbf{a}_{\mathcal{S}, \max }=\left(a_{\mathcal{S}, 1, \max }, a_{\mathcal{S}, 2, \max } \ldots, a_{\mathcal{S}, k, \max }\right)$, where $a_{\mathcal{S}, c, \min }=\max \left(0, r_{\mathcal{S}, c}-\left(n_{c}-n_{1, c}\right)\right)$ and $a_{\mathcal{S}, c, \max }=\min \left(n_{1, c}, r_{\mathcal{S}, c}\right)$ for each $c=1,2, \ldots, k$. Then, the minimum attainable P-value for the CMH test is given by:

$$
p_{\mathcal{S}, \min }=1-F_{\chi_{1}^{2}}\left(\max \left(T_{c m h}\left(\mathbf{a}_{\mathcal{S}, \min } \mid \mathbf{n}, \mathbf{n}_{\mathbf{1}}, \mathbf{r}_{\mathcal{S}}\right), T_{c m h}\left(\mathbf{a}_{\mathcal{S}, \max } \mid \mathbf{n}, \mathbf{n}_{\mathbf{1}}, \mathbf{r}_{\mathcal{S}}\right)\right)\right)
$$


In particular, this implies that $p_{\mathcal{S} \text {, min }}$ can be evaluated in $O(k)$ time, where $k$ is the number of categories for the categorical covariate $C$.

Proof Equation 8.29 can be rewritten as:

$$
T_{\mathrm{cmh}}\left(\mathbf{a}_{\mathcal{S}} \mid \mathbf{n}, \mathbf{n}_{\mathbf{1}}, \mathbf{r}_{\mathcal{S}}\right)=\frac{\left(a_{\mathcal{S}, \text { tot }}-\sum_{c=1}^{k} r_{\mathcal{S}, c} \frac{n_{1, c}}{n_{c}}\right)^{2}}{\sum_{c=1}^{k} \frac{r_{\mathcal{S}, c}}{n_{c}} \frac{n_{c}-r_{\mathcal{S}, c}}{n_{\mathcal{c}}} \frac{n_{c}-n_{1, c}}{n_{c}-1} n_{1, c}}
$$

where $a_{\mathcal{S} \text {,tot }}=\sum_{c=1}^{k} a_{\mathcal{S}, c}$ has been introduced. As described in Section 8.2.3, given fixed margins $n_{1, c}, r_{\mathcal{S}, c}$ and sample size $n_{c}$, each count $a_{\mathcal{S}, c}$ can only take values in the set $a_{\mathcal{S}, c} \in \llbracket a_{\mathcal{S}, c, \min }, a_{\mathcal{S}, c, \max } \rrbracket$, where $a_{\mathcal{S}, c, \min }=\max \left(0, r_{\mathcal{S}, c}-\left(n_{c}-n_{1, c}\right)\right)$ and $a_{\mathcal{S}, c, \min }=$ $\min \left(n_{1, c}, r_{\mathcal{S}, c}\right)$. Thus, $a_{\mathcal{S}, \text { tot }} \in \llbracket a_{\mathcal{S}, \text { tot,min }}, a_{\mathcal{S}, \text { tot,max }} \rrbracket$, where $a_{\mathcal{S}, \text { tot,min }}=\sum_{c=1}^{k} a_{\mathcal{S}, c, \text { min }}$ and $a_{\mathcal{S}, \text { tot, } \max }=\sum_{c=1}^{k} a_{\mathcal{S}, c, \max }$. According to Equation 8.32, $T_{\mathrm{cmh}}\left(\mathbf{a}_{\mathcal{S}} \mid \mathbf{n}, \mathbf{n}_{\mathbf{1}}, \mathbf{r}_{\mathcal{S}}\right)$ will be maximized and, hence, $p_{\mathrm{cmh}}\left(\mathbf{a}_{\mathcal{S}} \mid \mathbf{n}, \mathbf{n}_{1}, \mathbf{r}_{\mathcal{S}}\right)$ minimized, either when $a_{\mathcal{S} \text {, tot }}=a_{\mathcal{S}, \text { tot,min }}$ or $a_{\mathcal{S} \text {,tot }}=a_{\mathcal{S}, \text { tot,max }}$. Equivalently, this occurs when $\mathbf{a}_{\mathcal{S}}=\mathbf{a}_{\mathcal{S}, \min }$ or $\mathbf{a}_{\mathcal{S}}=\mathbf{a}_{\mathcal{S}, \max }$ concluding the proof.

\subsubsection{A Search Space Pruning Condition for the CMH Test}

In Section 8.3.2, a search space pruning condition valid for Pearson's $\chi^{2}$ test and Fisher's exact test was derived. The fundamental principle of that pruning criterion is that, for fixed $n_{1}$ and $n$, the minimum attainable $P$-value $p_{\mathcal{S} \text {, min }}$ for these test statistics is a monotonically decreasing function of $r_{\mathcal{S}}$ in the range $r_{\mathcal{S}} \in \llbracket 0, \min \left(n_{1}, n-n_{1}\right) \rrbracket$. This implies that, if a pattern $\mathcal{S}$ is untestable at level $\delta$ and satisfies $r_{\mathcal{S}} \leq \min \left(n_{1}, n-n_{1}\right)$, all its descendants $\mathcal{S}^{\prime} \in$ Children $(\mathcal{S})$ in the pattern enumeration tree will be untestable at level $\delta$ as well and can be pruned from the search space. In this section, we will propose an equivalent pruning condition for the $\mathrm{CMH}$ test.

The first key observation is that, for fixed $\mathbf{n}$ and $\mathbf{n}_{\mathbf{1}}$, the minimum attainable $P$ value for the $\mathrm{CMH}$ test is a multivariate function of $k$ variables, $r_{\mathcal{S}, 1}, r_{\mathcal{S}, 2}, \ldots, r_{\mathcal{S}, k}$. We will denote this by $p_{\mathcal{S}, \min }\left(\mathbf{r}_{\mathcal{S}}\right)$, where the dependence of $p_{\mathcal{S}, \min }$ on $\mathbf{n}$ and $\mathbf{n}_{\mathbf{1}}$ is kept implicit to avoid cluttering the notation. By applying the apriori property of pattern mining (see Proposition 8.2), it can be shown that $\mathcal{S}^{\prime} \in \operatorname{Children}(\mathcal{S}) \Rightarrow r_{\mathcal{S}^{\prime}, c} \leq$ $r_{\mathcal{S}, c} \forall c=1,2, \ldots, k$. Using identical arguments as in Section 8.3.2, a majority of patterns $\mathcal{S} \in \mathcal{M}$ in the search space of candidate patterns will be relatively rare, satisfying $r_{\mathcal{S}, c} \leq \min \left(n_{1, c}, n_{c}-n_{1, c}\right)$ for all $c=1,2, \ldots, k$. This naturally leads to the fundamental question of all $c=1,2, \ldots, k$. This naturally leads to the fundamental question of whether $\mathbf{r}_{\mathcal{S}^{\prime}} \leq \mathbf{r}_{\mathcal{S}} \Rightarrow p_{\mathcal{S}, \min }\left(\mathbf{r}_{\mathcal{S}^{\prime}}\right) \geq p_{\mathcal{S}, \min }\left(\mathbf{r}_{\mathcal{S}}\right)$ given that $r_{\mathcal{S}, c} \leq \min \left(n_{1, c}, n_{c}-n_{1, c}\right) \forall c=$ $1,2, \ldots, k{ }^{14}$ If the answer to this question was affirmative, a pruning condition entirely analogous to the one used for Pearson's $\chi^{2}$ test and Fisher's exact test would also be valid for the $\mathrm{CMH}$ test.

Unfortunately, it is easy to come up with non-pathological counterexamples which show that this property does not hold in general. As an example, Figure 8.12 depicts the minimum attainable $P$-value $p_{\mathcal{S} \text {, min }}$ for the $\mathrm{CMH}$ test in a problem with $k=2$

14 The notation $\mathbf{r}_{\mathcal{S}^{\prime}} \leq \mathbf{r}_{\mathcal{S}}$ was introduced as shorthand for $r_{\mathcal{S}^{\prime}, c} \leq r_{\mathcal{S}, c} \forall c=1,2, \ldots, k$ 


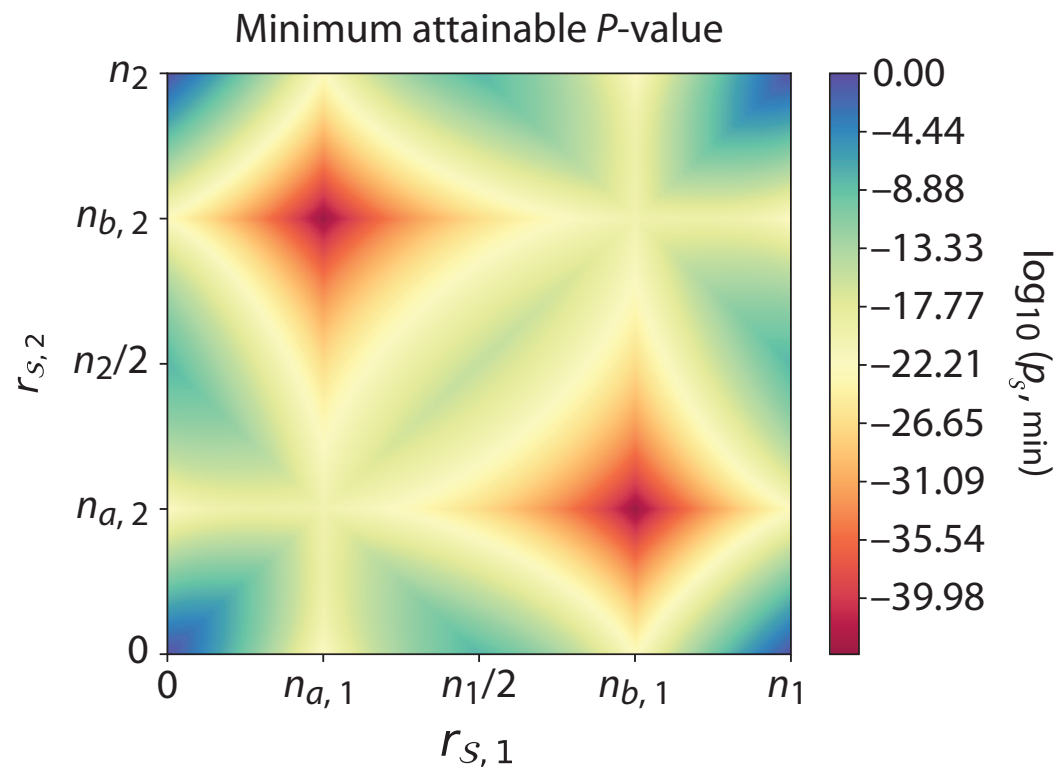

(a)

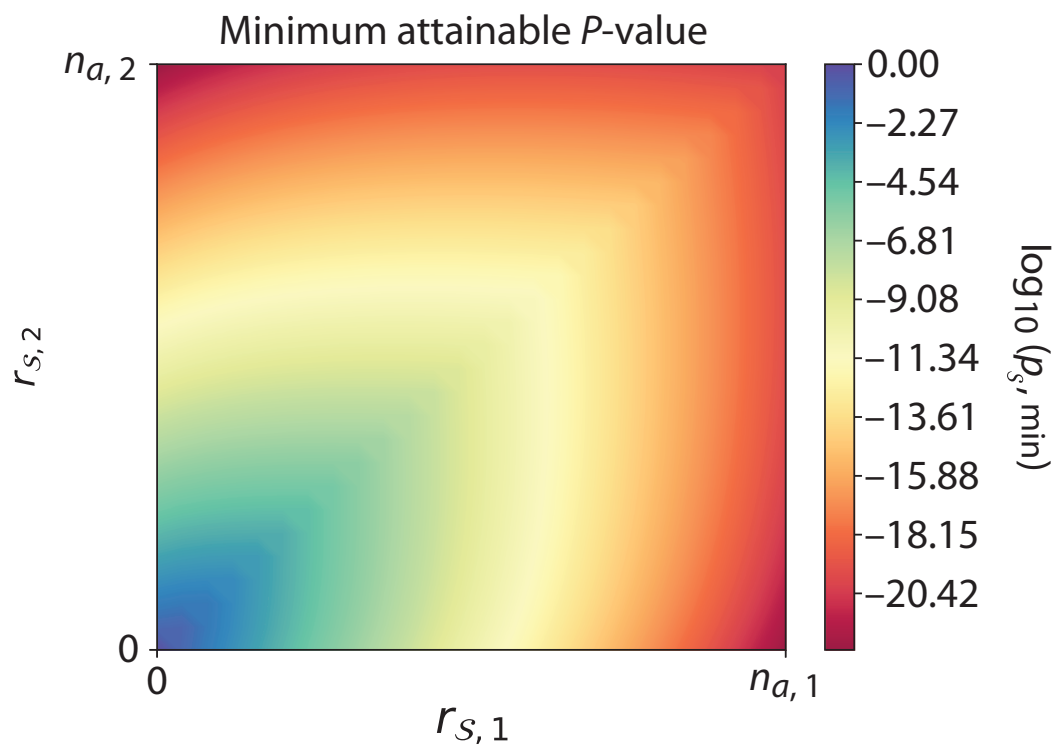

(b)

Figure 8.12: Visualization of the minimum attainable $P$-value $p_{\mathcal{S}, \min }$ for the $\mathrm{CMH}$ test in a problem with $k=2$ categories for the covariate. In this example, $n_{1}=n_{2}=100$ and

$n_{1,1}=25, n_{1,2}=75$. Thus, category $c=1$ has a class ratio of $1 / 4$ while category $c=2$ has a class ratio of 3/4. (a) Minimum attainable $P$-value $p_{\mathcal{S}, \min }\left(\mathbf{r}_{\mathcal{S}}\right)$ over the entire domain $\llbracket 0, n_{1} \rrbracket \times \llbracket 0, n_{2} \rrbracket$. (b) Minimum attainable $P$-value $p_{\mathcal{S}, \min }\left(\mathbf{r}_{\mathcal{S}}\right)$ over the region $\llbracket 0, n_{a, 1} \rrbracket \times \llbracket 0, n_{a, 2} \rrbracket$, where $n_{a, 1}=\min \left(n_{1,1}, n_{1}-n_{1,1}\right), n_{a, 2}=\min \left(n_{1,2}, n_{2}-n_{1,2}\right)$, $n_{b, 1}=\max \left(n_{1,1}, n_{1}-n_{1,1}\right)$ and $n_{b, 2}=\max \left(n_{1,2}, n_{2}-n_{1,2}\right)$. 
categories for the covariate. In particular, Figure 8.12 (b) illustrates the behavior of $p_{\mathcal{S}, \min }$ as a function of $r_{\mathcal{S}, 1}$ and $r_{\mathcal{S}, 2}$ over the region $\llbracket 0, \min \left(n_{1,1}, n_{1}-n_{1,1}\right) \rrbracket \times$ $\llbracket 0, \min \left(n_{1,2}, n_{2}-n_{1,2}\right) \rrbracket$. While the function is approximately monotonic when $r_{\mathcal{S}, 1}$ and $r_{\mathcal{S}, 2}$ are both sufficiently far from zero, $p_{\mathcal{S}, \min }\left(\mathbf{r}_{\mathcal{S}}\right)$ is not monotonically decreasing when one of its arguments is small enough, as can be appreciated from the level curves. This has profound implications for the development of a valid pruning criterion, as in principle there is no simple way to make a statement about the minimum attainable $P$-value $p_{\mathcal{S}^{\prime} \text {,min }}$ of a pattern $\mathcal{S}^{\prime} \in$ Children $(\mathcal{S})$ based on $p_{\mathcal{S} \text {, min }}$ and $\mathbf{r}_{\mathcal{S}}$ alone.

In order to solve this problem, the authors in [20] propose using a monotonically decreasing lower bound on the minimum attainable $P$-value as a surrogate in the pruning criterion. Borrowing their terminology, we refer to this surrogate as the lower envelope of the minimum attainable $P$-value.

Definition 8.7. Let $\mathcal{S} \in \mathcal{M}$ be a pattern satisfying $r_{\mathcal{S}, c} \leq \min \left(n_{1, c}, n_{c}-n_{1, c}\right)$ $\forall c=1,2, \ldots, k$. The lower envelope of the minimum attainable $P$-value $p_{\mathcal{S} \text {, min }}$ is defined as:

$$
\tilde{p}_{\mathcal{S}, \min }=\min _{\mathcal{S}^{\prime} \supseteq \mathcal{S}} p_{\mathcal{S}, \min }
$$

Note that, as a consequence of the apriori property of pattern mining, $\tilde{p}_{\mathcal{S} \text {, min }}$ can be equivalently defined as:

$$
\tilde{p}_{\mathcal{S}, \min }\left(\mathbf{r}_{\mathcal{S}}\right)=\min _{\mathbf{r}_{\mathcal{S}^{\prime}} \leq \mathbf{r}_{\mathcal{S}}} p_{\mathcal{S}^{\prime}, \min }\left(\mathbf{r}_{\mathcal{S}^{\prime}}\right),
$$

where the dependence of the minimum attainable $P$-value $p_{\mathcal{S} \text {, min }}$ and its lower envelope $\tilde{p}_{\mathcal{S}, \min }$ on $\mathbf{r}_{\mathcal{S}}$ has been made explicit.

Intuitively, $\tilde{p}_{\mathcal{S}, \min }\left(\mathbf{r}_{\mathcal{S}}\right)$ is defined as the tightest lower bound on the minimum attainable $P$-value $p_{\mathcal{S} \text {, min }}$, hence the term "lower envelope," that satisfies $\mathbf{r}_{\mathcal{S}^{\prime}} \leq \mathbf{r}_{\mathcal{S}} \Rightarrow$ $\tilde{p}_{\mathcal{S}, \min }\left(\mathbf{r}_{\mathcal{S}^{\prime}}\right) \geq \tilde{p}_{\mathcal{S}, \min }\left(\mathbf{r}_{\mathcal{S}}\right)$. This notion is illustrated in Figure 8.13 with a conceptual example.

The lower envelope $\tilde{p}_{\mathcal{S} \text {, min }}$ is a lower bound of the minimum attainable $P$-value $p_{\mathcal{S} \text {, min }}$ by construction. Also, the fact that $\tilde{p}_{\mathcal{S}, \min }\left(\mathbf{r}_{\mathcal{S}}\right)$ is monotonically decreasing on $\mathbf{r}_{\mathcal{S}}$, i.e., that $\mathbf{r}_{\mathcal{S}^{\prime}} \leq \mathbf{r}_{\mathcal{S}} \Rightarrow \tilde{p}_{\mathcal{S}, \min }\left(\mathbf{r}_{\mathcal{S}^{\prime}}\right) \geq \tilde{p}_{\mathcal{S}, \min }\left(\mathbf{r}_{\mathcal{S}^{\prime}}\right) \geq \tilde{p}_{\mathcal{S}, \min }\left(\mathbf{r}_{\mathcal{S}}\right)$, is a direct consequence of the way $\tilde{p}_{\mathcal{S} \text {, min }}$ is defined. If $\mathbf{r}_{\mathcal{S}^{\prime}} \leq \mathbf{r}_{\mathcal{S}}$, then $\tilde{p}_{\mathcal{S}, \min }\left(\mathbf{r}_{\mathcal{S}^{\prime}}\right)=\min _{\mathbf{r}_{\mathcal{S}^{\prime \prime}} \leq \mathbf{r}_{\mathcal{S}^{\prime}}} p_{\mathcal{S}^{\prime \prime}, \min }\left(\mathbf{r}_{\mathcal{S}^{\prime \prime}}\right) \leq$ $\min _{\mathbf{r}_{\mathcal{S}^{\prime \prime}} \leq \mathbf{r}_{\mathcal{S}}} p_{\mathcal{S}^{\prime \prime}, \min }\left(\mathbf{r}_{\mathcal{S}^{\prime \prime}}\right)=p_{\mathcal{S}^{\prime \prime}, \min }\left(\mathbf{r}_{\mathcal{S}^{\prime \prime}}\right)=\tilde{p}_{\mathcal{S}, \min }\left(\mathbf{r}_{\mathcal{S}}\right)$ since $\mathbf{r}_{\mathcal{S}^{\prime \prime}} \leq \mathbf{r}_{\mathcal{S}^{\prime}} \subseteq \mathbf{r}_{\mathcal{S}^{\prime \prime}} \leq \mathbf{r}_{\mathcal{S}}$. These two properties of $\tilde{p}_{\mathcal{S}}$, min allow proposing the following search space pruning criterion for the $\mathrm{CMH}$ test.

Proposition 8.8 (Pruning criterion for the Cochran-Mantel-Haenszel (CMH) test) Let $\mathcal{S} \in \mathcal{M}$ be a pattern satisfying:

1. $\tilde{p}_{\mathcal{S}, \min }>\hat{\delta}_{t a r}$, i.e., the lower envelope of the minimum attainable P-value is larger than $\hat{\delta}_{\text {tar }}$

2. $r_{\mathcal{S}, c} \leq n_{a, c} \forall c \in\{1, \ldots, k\}$, where $n_{a, c}=\min \left(n_{1, c}, n_{c}-n_{1, c}\right)$ 


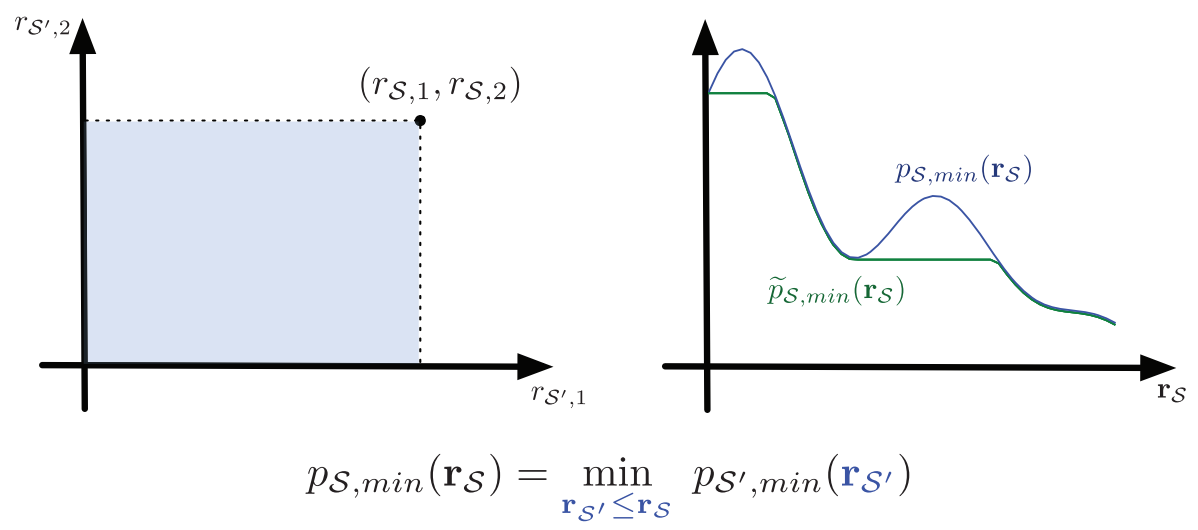

Figure 8.13: Illustration of the lower envelope $\tilde{p}_{\mathcal{S}, \text { min }}$ of the minimum attainable $P$-value $p_{\mathcal{S}, \min }$. To evaluate $\tilde{p}_{\mathcal{S}, \min }\left(\mathbf{r}_{\mathcal{S}}\right)$, the minimum of $p_{\mathcal{S}^{\prime}, \min }\left(\mathbf{r}_{\mathcal{S}^{\prime}}\right)$ over the region $\mathbf{r}_{\mathcal{S}^{\prime}} \leq \mathbf{r}_{\mathcal{S}}$ needs to be computed. In this example, this corresponds to minimizing $p_{\mathcal{S}^{\prime}, \min }\left(\mathbf{r}_{\mathcal{S}^{\prime}}\right)$ over the region shaded in blue on the left of the figure. As a result of this definition, $\tilde{p}_{\mathcal{S}, \min }\left(\mathbf{r}_{\mathcal{S}}\right)$ is the tightest lower bound on $p_{\mathcal{S}, \min }\left(\mathbf{r}_{\mathcal{S}}\right)$ that satisfies $\mathbf{r}_{\mathcal{S}^{\prime}} \leq \mathbf{r}_{\mathcal{S}} \Rightarrow \tilde{p}_{\mathcal{S}, \min }\left(\mathbf{r}_{\mathcal{S}^{\prime}}\right) \geq \tilde{p}_{\mathcal{S}, \min }\left(\mathbf{r}_{\mathcal{S}}\right)$. This is illustrated, along a one-dimensional slice, on the right of the figure.

Then, $p_{\mathcal{S}^{\prime}, \text { min }}>\hat{\delta}_{\text {tar }} \geq \delta_{\text {tar }}$ for all $\mathcal{S}^{\prime} \in \operatorname{Children}(\mathcal{S})$. Hence, all descendants of pattern $\mathcal{S}$ can be pruned from the enumeration tree. In conclusion, when using the CMH test, pruning_condition $\left(\mathcal{S}, \hat{\delta}_{\text {tar }}\right)$ in Line 15 of Algorithm 8.2 evaluates to true if $r_{\mathcal{S}, c} \leq$ $n_{a, c} \forall c \in\{1, \ldots, k\}$ and $\tilde{p}_{\mathcal{S}, \min }>\hat{\delta}_{\text {tar }}$.

Proof If $\mathcal{S}^{\prime} \in$ Children $(\mathcal{S}), \mathbf{r}_{\mathcal{S}^{\prime}} \leq \mathbf{r}_{\mathcal{S}}$ by the apriori property of pattern mining. Hence, as a consequence of the monotonicity of $\tilde{p}_{\mathcal{S}, \min }\left(\mathbf{r}_{\mathcal{S}}\right), \widetilde{p}_{\mathcal{S}^{\prime}, \min }\left(\mathbf{r}_{\mathcal{S}^{\prime}}\right) \geq \tilde{p}_{\mathcal{S}, \min }\left(\mathbf{r}_{\mathcal{S}}\right)$. Since the lower envelope $\tilde{p}_{\mathcal{S} \text {, min }}$ is a lower bound on the minimum attainable P-value $p_{\mathcal{S} \text {, min }}$ and on the minimum attainable P-value $p_{\mathcal{S} \text {,min }}$ and $\tilde{p}_{\mathcal{S} \text {, min }}>\hat{\delta}_{\text {tar }}$ by assumption (i), it follows that $p_{\mathcal{S}^{\prime}, \min }>\hat{\delta}_{\text {tar }}$. Finally, the fact that $\hat{\delta}_{\text {tar }} \geq \delta_{\text {tar }}$ at any point during the execution of Algorithm 8.2 concludes the proof.

The resulting pruning condition for the $\mathrm{CMH}$ test is mostly analogous to the pruning criterion for Pearson's $\chi^{2}$ test and Fisher's exact test in Proposition 8.5. However, the minimum attainable $P$-value $p_{\mathcal{S} \text {, min }}$ in condition (i), $p_{\mathcal{S} \text {, min }}>\hat{\delta}_{\text {tar }}$, is substituted by the lower envelope $\tilde{p}_{\mathcal{S} \text {, min }}$. This allows circumventing the difficulties which arise

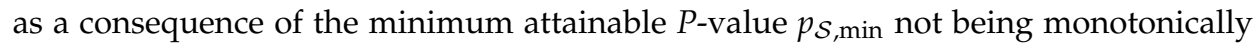
decreasing for the $\mathrm{CMH}$ test. Note that while the concept of lower envelope of the minimum attainable $P$-value was introduced in [20] in the context of the $\mathrm{CMH}$ test, the same idea could be used for other discrete tests statistics with a non-monotonic minimum attainable $P$-value function. This might help develop new applications of significant pattern mining by using domain-specific test statistics.

Nevertheless, an important aspect that remains to be considered is how to efficiently evaluate $\tilde{p}_{\mathcal{S}, \min }$. A straightforward application of Definition 8.7 implies that a combinatorial optimization problem needs to be solve each time $\tilde{p}_{\mathcal{S} \text {, min }}$ is to be computed. Since the pruning condition is evaluated for every single pattern $\mathcal{S} \in \mathcal{M}$ that is enumerated along the execution the algorithm, this would entail an unfeasible computational overhead. In [20] the authors propose a procedure, specific to the $\mathrm{CMH}$ 
test, that allows computing $\tilde{p}_{\mathcal{S} \text {, min }}$ with $O(k \log k)$ operations, where $k$ is the number of categories for the covariate. Consequently, $\tilde{p}_{\mathcal{S} \text {, min }}$ can be evaluated almost as fast as $p_{\mathcal{S} \text {, min }}$ for the $\mathrm{CMH}$ test, making the entire idea practical. Nonetheless, deriving a more general procedure to efficiently evaluate $\tilde{p}_{\mathcal{S}, \min }$, regardless of the test statistic of choice, is still an open problem.

An alternative approach to account for the effect of a confounding categorical covariate in significant pattern mining was developed by [52]. The main difference with respect to the method described in this section lies in the choice of test statistic. Rather than making use of the $\mathrm{CMH}$ test, the approach in [52] uses an exact formulation of logistic regression. By exploiting the discrete nature of the data, this formulation allows to obtain exact $P$-values for the logistic regression model, avoiding the need for large sample approximations. For additional details, we refer the reader to the original article [52].

\subsection{Summary and Outlook}

Data analysis in computational biology and healthcare is a challenging task. In order to discover relevant biomarkers from omics and clinical data, the association of a large number of candidate markers with a phenotypic trait of interest needs to be assessed. The small sample size of most available datasets, relative to the number of features, has deterred the search for combinatorial feature interactions and other high-order patterns as potential biomarkers. In this chapter, significant pattern mining has been introduced as a family of machine learning algorithms to systematically explore such high-order structures and assess their statistical association. These techniques are a useful complement for the univariate analyses and linear models typically used for biomarker discovery, which cannot discover such non-linear signals in the data.

At the core of significant pattern mining is the concept of testability [38], introduced by R. E. Tarone in 1990. This is the fact that, when testing statistical associations in discrete data, there exists a minimum attainable $P$-value strictly larger than zero. Consequently, it can be shown that many candidate patterns cannot achieve significance or cause a false positive, leading to an elegant way to solve the statistical and computational challenges that significant pattern mining entails.

Recent advances in the field have focused on solving some of the core limitations of the significant pattern mining algorithm that first advocated the use of Tarone's method. One crucial aspect, not exploited by this algorithm, is the inherent redundancy between candidate patterns. Several novel methods have been proposed to model these dependencies between patterns, allowing to obtain a less conservative significance threshold and gain statistical power. Another fundamental limitation of the original approach is its inability to correct for observed confounding factors such as age, gender, or population structure. These are ubiquitous in biomedical data analysis applications and, if unaccounted for, could give rise to a large number of spurious discoveries. Recently developed significant pattern mining algorithms extend the original approach to incorporate such covariate factors into the model without sacrificing statistical power nor computational efficiency, effectively overcoming this limitation. 
Table 8.1: A summary of available significant pattern mining tools.

\begin{tabular}{|lcl|}
\hline Algorithm & Reference & Highlights \\
\hline LAMP, LAMP2 .01 & {$[15,16]$} & $\begin{array}{l}\text { First use of Tarone's method in significant } \\
\text { itemset mining } \\
\text { LAMPLINK }\end{array}$ \\
MP-LAMP & {$[53]$} & $\begin{array}{l}\text { GWAS-oriented user interface for high-order } \\
\text { epistasis search }\end{array}$ \\
\hline WY-light & {$[54]$} & $\begin{array}{l}\text { Distributed significant itemset mining } \\
\text { algorithm for supercomputers }\end{array}$ \\
FACS $^{4}$ & {$[19]$} & $\begin{array}{l}\text { Permutation-testing based significant } \\
\text { itemset and subgraph mining } \\
\text { FAIS, FastCMH }\end{array}$ \\
& {$[20]$} & $\begin{array}{l}\text { Corrects for a categorical covariate in } \\
\text { significant itemset mining } \\
\text { Region-wise GWAS, with optional } \\
\text { correction for a categorical covariate }\end{array}$ \\
\hline
\end{tabular}

Notes:

${ }^{1}$ Available from http://a-terada.github.io/lamp.

2 Available from http:/ /a-terada.github.io/lamplink.

3 Available from http:/ / github.com/tsudalab/mp-lamp.

${ }^{4}$ Available from http://significant-patterns.org.

\subsubsection{Software}

There exist software tools implementing many of the approaches discussed in this chapter, allowing practitioners to use significant pattern mining to analyze their data (see Table 8.1).

Interested users can obtain both an implementation of LAMP [15], the first pattern mining algorithm using Tarone's method, as well as of LAMP 2.0 [16], a followup approach that improves its computational efficiency. Recently, the same authors have also implemented LAMPLINK [53], a toolbox that considerably extends the input/output format of these algorithms to greatly facilitate their use in genomewide association studies (GWASs). MP-LAMP [54], a parallel implementation of the LAMP 2.0 algorithm, suitable for execution in distributed environments such as computing clusters, is also at the users' disposal. Similarly, users can download implementations of: (1) WY-light and FACS, the significant pattern mining algorithms described in [19] and [20] and (2) FAIS and FastCMH, methods that use significant pattern mining to aggregate weak effects within any genomic region in genome-wide association studies [55, 49]. All these software packages and their main defining characteristics are summarized in Table 8.1. The algorithm names in this table contain hyperlinks to their respective software repositories.

\subsubsection{Outlook}

The development of methods to correct for the multiple hypothesis testing problem in pattern mining, without resorting to arbitrary limits on pattern size, is a relatively 
recent field of study. As a consequence, many open problems and future research directions remain to be explored.

In this chapter, family-wise error rate (FWER) control has been the central tool to account and correct for the multiple hypothesis testing problem. However, statisticians have produced a wealth of alternative criterions for this purpose. For instance, false discovery rate (FDR) control [56] has been established as a popular alternative to FWER control in the biomedical field. Unlike FWER control, which aims at keeping the probability of producing any false positives low, FDR control aims at upper bounding the expected proportion of false discoveries among all reported findings. An FDR-controlling procedure is effectively allowed to produce false positives with high probability, as long as it also yields sufficiently many true positives. This makes FDR control considerably less stringent than FWER control, leading to a gain of statistical power that is useful in applications for which signals are weak, such as computational biology and healthcare, at the price of increasing the expected number of false positives. Most FDR-controlling procedures require assumptions about the joint distribution of all test statistics corresponding to null hypotheses, often in the form of independence or weak dependence assumptions. Due to the complex inter-dependencies between candidate patterns, these assumptions are hard to justify in the context of significant pattern mining, explaining the fact that most existing methods have focused on FWER control. Extending state-of-the-art significant pattern mining algorithms to perform FDR control is an exciting avenue for future research. Another recently proposed alternative to FWER control is selective inference [57], which aims at developing procedures to properly quantify statistical associations after having performed model selection. Recent work [58] has begun exploring how to apply principles of selective inference to pattern mining, opening yet another interesting research direction.

Discrete data is at the core of all significant pattern mining algorithms discussed in this chapter. While this is sufficient to cover many applications of interest, it would be desirable to develop alternative methods to find high-order feature interactions in datasets with continuous features. In some occasions, a real-valued feature can be binarized without losing too much information, allowing the use of existing significant pattern mining algorithms to analyse the data after a discretization step. However, the need to binarize the data might be undesirable in some settings. Developing significant pattern mining tools that can handle continuous data is a particularly challenging problem as Tarone's concept of testability would, in principle, no longer apply. Solving this limitation remains a key topic for future work.

Finally, significant pattern mining methods are flexible in the way the search space of candidate patterns is defined. While most approaches discussed in this chapter aim at performing an exhaustive search, prior knowledge can be used to drastically reduce the number of candidate high-order structures under study. This would not only lead to improved computational efficiency and statistical power but would also make it easier for practitioners to interpret the results of the analysis. Deriving novel application-specific pattern mining algorithms, combining domain knowledge with the basic principles of general-purpose significant pattern mining methods, is a promising research direction for data analysis in computational biology and medicine. 


\subsection{Exercises}

8.1 Let $G$ and $Y$ be two random variables taking values in the finite alphabets $\mathcal{G}$ and $\mathcal{Y}$, respectively. Further assume that all marginal probabilities of $G$ and $Y$ are nonzero, that is, $\operatorname{Pr}(G=g)>0$ for all $g \in \mathcal{G}$ and $\operatorname{Pr}(Y=y)>0$ for all $y \in \mathcal{Y}$. Assuming that one of the three statements below is true, prove that the other two statements must hold as well.

(a) $\operatorname{Pr}(G=g, Y=y)=\operatorname{Pr}(G=g) \operatorname{Pr}(Y=y)$ for all $(g, y) \in \mathcal{G} \times \mathcal{Y}$.

(b) $\operatorname{Pr}(G=g \mid Y=y)=\operatorname{Pr}(G=g)$ for all $(g, y) \in \mathcal{G} \times \mathcal{Y}$.

(c) $\operatorname{Pr}(Y=y \mid G=g)=\operatorname{Pr}(Y=y)$ for all $(g, y) \in \mathcal{G} \times \mathcal{Y}$.

8.2 Let $G$ and $Y$ be two binary random variables. Suppose that there exists $(g, y) \in\{0,1\}^{2}$ such that $\operatorname{Pr}(G=g, Y=y)=\operatorname{Pr}(G=g) \operatorname{Pr}(Y=y)$. Prove that this implies that:

(a) $\operatorname{Pr}(G=1-g, Y=y)=\operatorname{Pr}(G=1-g) \operatorname{Pr}(Y=y)$.

(b) $\operatorname{Pr}(G=g, Y=1-y)=\operatorname{Pr}(G=g) \operatorname{Pr}(Y=1-y)$.

(c) $\operatorname{Pr}(G=1-g, Y=1-y)=\operatorname{Pr}(G=1-g) \operatorname{Pr}(Y=1-y)$.

8.3 Let $G$ and $Y$ be two binary random variables. Suppose that there exists $(g, y) \in\{0,1\}^{2}$ such that $\operatorname{Pr}(G=g \mid Y=y)=\operatorname{Pr}(G=g)$. Prove that this implies that $G$ and $Y$ are statistically independent random variables.

8.4 Consider a binary random variable $Y$ that satisfies $\operatorname{Pr}(Y=1)=p$. We say that $Y$ follows a Bernoulli distribution with success probability $p$, denoted by $\operatorname{Pr}(Y=y)=\operatorname{Bernoulli}(y \mid p)=p^{y}(1-p)^{1-y}$. Suppose that $n$ independent realizations of $Y$ are collected, leading to a sequence of $n$ i.i.d.

Bernoulli-distributed random variables $\left\{Y_{i}\right\}_{i=1}^{n}$, all of which have the same success probability $p$. Finally, define $Z=\sum_{i=1}^{n} Y_{i}$ to be an integer-valued random variable that describes the total number of "successes," i.e., realizations for which $Y_{i}=1$, out of all $n$ draws.

The goal of this exercise is to prove that $Z$ follows a binomial distribution with parameters $n$ and $p$, that is, to show that

$$
\operatorname{Pr}(Z=z \mid n, p)=\left(\begin{array}{l}
n \\
z
\end{array}\right) p^{z}(1-p)^{n-z}
$$

for any integer $z$ between 0 and $n$. The proof will make use of four intermediate steps:

(a) Show that the joint probability distribution of $\left\{Y_{i}\right\}_{i=1}^{n}$ can be expressed as

$$
\operatorname{Pr}\left(Y_{1}=y_{1}, \ldots, Y_{n}=y_{n}\right)=p^{\sum_{i=1}^{n} y_{i}}(1-p)^{n-\sum_{i=1}^{n} y_{i}}
$$

(b) Show that $\operatorname{Pr}(Z=z \mid n, p)$ and $\operatorname{Pr}\left(Y_{1}=y_{1}, \ldots, Y_{n}=y_{n}\right)$ are related by

$$
\operatorname{Pr}(Z=z \mid n, p)=\operatorname{Pr}\left(Y_{1}=y_{1}(z), \ldots, Y_{n}=y_{n}(z)\right)\left|\mathcal{Y}^{n}(z)\right|
$$


where $\mathcal{Y}^{n}(z)=\left\{\mathbf{y}=\left(y_{1}, \ldots, y_{n}\right) \mid z=\sum_{i=1}^{n} y_{i}\right\}$ is the set of all realizations $\mathbf{y}=\left(y_{1}, \ldots, y_{n}\right)$ of $\left\{Y_{i}\right\}_{i=1}^{n}$ that have exactly $z$ out of $n$ successes and $\mathbf{y}(z)=\left(y_{1}(z), \ldots, y_{n}(z)\right)$ is any realization in $\mathcal{Y}^{n}(z)$.

(c) Derive a closed-form expression for $\operatorname{Pr}\left(Y_{1}=y_{1}(z), \ldots, Y_{n}=y_{n}(z)\right)$.

(d) Derive a closed-form expression for $\left|\mathcal{Y}^{n}(z)\right|$.

Finally, show that the result readily follows from (a)-(d).

8.5 A group of researchers wishes to carry out a large-scale experiment to empirically validate a crucial, long-standing hypothesis in their field. The researchers are uncertain of the exact conditions in which the experiment should be performed. However, owing to the impact that a positive finding would have, they decide to exhaustively repeat the experiment $D=100$ times, using slightly different conditions on each occasion. They compute a $P$-value for each such repetition of the experiment and consider that a positive finding has occurred if the $P$-value is smaller than $\alpha=0.05$. To the researchers' excitement, for one specific set of experimental conditions, the $P$-value was indeed smaller than $\alpha=0.05$. Therefore, they conclude that these were the right conditions in which the experiment should be carried out, and prepare a publication in which they describe what they now deem to be the correct experimental setup, as well as the corresponding findings. Unfortunately, in the next years after their article was published, no other research group managed to replicate their results.

(a) Suppose that the set of $D=100 P$-values the researchers obtained are jointly statistically independent. Derive a closed-form expression for the exact FWER of their procedure as a function of $\alpha$, assuming the hypothesis they wish to validate were not true for any of the $D=100$ experimental conditions they considered.

(b) What is the corresponding FWER at $\alpha=0.05$ ? Should the failure to replicate their results come as a surprise?

(c) How would the results change if they had only attempted to test $D=15$ different experimental conditions?

(d) Based on the expression derived in (a), obtain the exact corrected significance threshold $\delta$ which would guarantee that the FWER is equal to $\alpha$. What is the corresponding value of $\delta$ for $\alpha=0.05$ ?

(e) What would be the corrected significance threshold obtained using the Bonferroni correction?

(f) Suppose now that the $D=100$ experiments were performed in five different laboratories, rendering the $P$-values for experiments carried out in the same laboratory no longer statistically independent. Can we guarantee that the corrected significance threshold obtained in (d) will control the FWER at level $\alpha$ ? What about the significance threshold obtained in (e)?

8.6 This exercise will explore informally the behavior of Tarone's method in the large sample size regime. 
(a) Show that the minimum attainable $P$-value function for Fisher's exact test $p_{\mathcal{S}, \min }\left(r_{\mathcal{S}}\right)$ can be approximated by $\left(\frac{n_{a}}{n}\right)^{r} \mathcal{S}$ provided that $n_{a}$ is large and that $r_{\mathcal{S}}<n_{a}$ is small.

The following approximations might be useful:

- $\log n ! \approx n \log n-n$ for sufficiently large $n$ (Stirling's approximation).

- $(y-x) \log (y-x) \approx y \log y-(1+\log y) x$ for $x \ll y$ (first order Taylor series of $(y-x) \log (y-x)$ around $x=0)$.

(b) Assuming that $\operatorname{Pr}\left(G_{\mathcal{S}}(X)=1\right)=p_{g}$ is constant and that $n_{a}$ and $n$ grow at the same rate, what does this imply for the behavior of the minimum attainable $P$-value as the sample size $n$ tends to infinity?

Note: Solutions are available to instructors at www.cambridge.org/bionetworks.

\subsection{Acknowledgments and Funding}

We thank Damian Roqueiro and Katharina Heinrich for helpful comments, discussions and helping proofread this manuscript. This work was funded in part by the SNSF Starting Grant "Significant Pattern Mining" (KB, FLL) and the Marie Curie ITN MLPM2012, Grant No. 316861 (KB, FLL).

\section{References}

[1] Listgarten J, Lippert C, Kadie C. M, et al. Improved linear mixed models for genome-wide association studies. Nature Methods, 2012;9(6):525-526.

[2] Tibshirani R. Regression shrinkage and selection via the lasso. Journal of the Royal Statistical Society. Series B (Statistical Methodology), 1996;58(1): 267-288.

[3] H. Zou and T. Hastie, Regularization and variable selection via the elastic net. Journal of the Royal Statistical Society: Series B (Statistical Methodology), 2005;67(2):301-320.

[4] Bondell HD, Reich BJ. Simultaneous regression shrinkage, variable selection, and supervised clustering of predictors with Oscar. Biometrics, 2008;64(1):115-123.

[5] Li C, Li H. Network-constrained regularization and variable selection for analysis of genomic data. Bioinformatics, 2008;24(9):1175-1182.

[6] Kim S, Sohn KA, Xing EP. A multivariate regression approach to association analysis of a quantitative trait network. Bioinformatics, 2009;25(12):i204-i212. DOI: http://dx.doi.org/10.1093/bioinformatics/btp218.

[7] Azencott CA, Grimm D, Sugiyama M, Kawahara Y, Borgwardt K. Efficient network-guided multi-locus association mapping with graph cuts.

Bioinformatics, 2013;29(13):i171-i179. 
[8] Welter D. MacArthur J, Morales J. et al. The NHGRI GWAS catalog: A curated resource of snp-trait associations. Nucleic Acids Research, 2014;42(D1):D1001-D1006.

[9] Visscher PM, Brown MA, McCarthy MI, Yang J. Five years of GWAS discovery. The American Journal of Human Genetics, 2012;90(1):7-24.

[10] Manolio TA, Collins FS, Cox N J, et al. Finding the missing heritability of complex diseases. Nature, 2009;461(7265):747-753.

[11] Zuk O, Hechter E, Sunyaev SR, Lander ES. The mystery of missing heritability: Genetic interactions create phantom heritability. Proceedings of the National Academy of Sciences, 2012;109(4):1193-1198.

[12] Hemani G, Shakhbazov K, Westra HJ, et al. Detection and replication of epistasis influencing transcription in humans. Nature, 2014;508(7495):249-253.

[13] Forsberg SK, Bloom JS, Sadhu MJ, Kruglyak L, Carlborg O. Accounting for genetic interactions improves modeling of individual quantitative trait phenotypes in yeast. Nature Genetics, 2017;49(4):497-503.

[14] MacKay DJ. Information Theory, Inference and Learning Algorithms. Cambridge University Press, 2003.

[15] Terada A, Okada-Hatakeyama M, Tsuda K, Sese J. Statistical significance of combinatorial regulations. Proceedings of the National Academy of Sciences, 2013;110(32):12996-13001.

[16] Minato SI, Uno T, Tsuda K, Terada A, Sese J. A fast method of statistical assessment for combinatorial hypotheses based on frequent itemset enumeration. in Joint European Conference on Machine Learning and Knowledge Discovery in Databases. Springer;2014; pp. 422-436.

[17] Sugiyama M, Llinares-Lopez F, Kasenburg N, Borgwardt K. Significant subgraph mining with multiple testing correction. In Proceedings of the 2015 SIAM International Conference on Data Mining 4, SIAM;2015, pp. 37-45.

[18] Terada A, Tsuda K, Sese J. Fast westfall-young permutation procedure for combinatorial regulation discovery. In 2013 IEEE International Conference on Bioinformatics and Biomedicine, 12. IEEE BIBM;2013, pp. 153-158.

[19] Llinares-Lopez F, Sugiyama M, Papaxanthos L, Borgwardt K. Fast and memory-efficient significant pattern mining via permutation testing. In Proceedings of the 21st ACM SIGKDD International Conference on Knowledge Discovery and Data Mining. ACM;2015, pp. 725-734.

[20] Papaxanthos L, Llinares-Lopez F, Bodenham D, Borgwardt K. Finding significant combinations of features in the presence of categorical covariates. In Jordan MI, LeCun Y, Solla SA, eds., Advances in Neural Information Processing Systems, MIT Press;2016, pp. 2271-2279.

[21] Todeschini R, Consonni V. Handbook of Molecular Descriptors. John Wiley \& Sons;2008.

[22] Bullmore E, Sporns O. Complex brain networks: graph theoretical analysis of structural and functional systems. Nature Reviews Neuroscience, 2009;10(3): 186-198. 
[23] van den Heuvel MP, Mandl RC, Stam CJ, Kahn RS, Pol, HEH. Aberrant frontal and temporal complex network structure in schizophrenia: A graph theoretical analysis. Journal of Neuroscience; 2010;30(47):15915-15926.

[24] Bertsekas DP, Tsitsiklis JN. Introduction to Probability. Athena Scientific; 2002.

[25] Pearson K. X. On the criterion that a given system of deviations from the probable in the case of a correlated system of variables is such that it can be reasonably supposed to have arisen from random sampling. The London, Edinburgh, and Dublin Philosophical Magazine and Journal of Science, 1900;50(302):157-175.

[26] Fisher RA. On the interpretation of $\chi^{2}$ from contingency tables, and the calculation of p. Journal of the Royal Statistical Society, 1922;85(1):87-94.

[27] Shaffer JP. Multiple hypothesis testing. Annual Review of Psychology, 1995;46(1):561-584.

[28] Dmitrienko A, Tamhane AC, Bretz F. Multiple testing problems in pharmaceutical statistics. Chapman and Hall/CRC, 2009.

[29] Noble WS. How does multiple testing correction work? Nature Biotechnology, 2009;27(12);1135-1137.

[30] Bonferroni CE. Teoria statistica delle classi e calcolo delle probabilita. Libreria Internazionale Seeber; 1936.

[31] Dunn OJ. Estimation of the medians for dependent variables. The Annals of Mathematical Statistics, 1959;30(1):192-197.

[32] Bay SD, Pazzani MJ. Detecting group differences: Mining contrast sets. Data Mining and Knowledge Discovery, 2001;5(3):213-246.

[33] Hamalainen W. Statapriori: An efficient algorithm for searching statistically significant association rules. Knowledge and Information Systems, 2010;23(3):373-399.

[34] Zimmermann A, Nijssen S. Supervised Pattern Mining and Applications to Classification. Springer;2014, pp. 425-442. DOI: / / doi.org/10.1007/978-3-319-07821-2 17.

[35] Webb GI. Discovering significant rules. In Proceedings of the 12th ACM SIGKDD International Conference on Knowledge Discovery and Data Mining. ACM;2006, pp. 434-443.

[36] —. Discovering significant patterns. Machine Learning, 2007;68(1):1-33.

[37] _ Layered critical values: A powerful direct-adjustment approach to discovering significant patterns. Machine Learning, 2008;71(2-3): 307-323.

[38] Tarone RE. A modified Bonferroni method for discrete data. Biometrics, 1990;46(2):515-522.

[39] Aggarwal CC, Han J. Frequent Pattern Mining. Springer;2014.

[40] Zaki MJ, Parthasarathy S, Ogihara M, Li W. New algorithms for fast discovery of association rules. In Proceedings of the Third International Conference on 
Knowledge Discovery and Data Mining. ACM;1997, pp. 283-286. Available at www.aaai.org/Library/KDD/1997/kdd97-060.php.

[41] Han J, Pei J, Yin Y. Mining frequent patterns without candidate generation. In Proceedings of the 2000 ACM SIGMOD International Conference on Management of Data. ACM;2000, pp. 1-12. DOI://doi.acm.org/10.1145/342009.335372.

[42] Uno T, Kiyomi M, Arimura H. LCM ver. 2: Efficient mining algorithms for frequent/closed/maximal itemsets. In Proceedings of IEEE ICDM'04 Workshop FIMI'04. IEEE;2004.

[43] Borgelt C. Frequent item set mining. Wiley Interdisciplinary Reviews: Data Mining and Knowledge Discovery, 2012;2(6):437-456.

[44] Yan X, Han J. gSpan: Graph-based substructure pattern mining. In IEEE International Conference on Data Mining. IEEE;2002, pp. 721-724.

[45] Nijssen S, Kok JN. The gaston tool for frequent subgraph mining. Electronic Notes in Theoretical Computer Science, 2005;127(1):77-87.

[46] Jiang C, Coenen F, Zito M. A survey of frequent subgraph mining algorithms. The Knowledge Engineering Review, 2013;28(1):75-105.

[47] Westfall PH, Young SS. Resampling-Based Multiple Testing: Examples and Methods for $p$-Value Adjustment, Wiley Series in Probability and Statistics, vol. 279. John Wiley \& Sons;1993.

[48] Vilhjalmsson BJ, Nordborg M. The nature of confounding in genome-wide association studies. Nature Reviews Genetics, 2013;14(1):1-2.

[49] Llinares-Lopez F, Papaxanthos L, Bodenham D, et al. Genome-wide genetic heterogeneity discovery with categorical covariates. Bioinformatics, 2017;33(12):i1820i1828,.

[50] Dering C, Hemmelmann C, Pugh E, Ziegler A. Statistical analysis of rare sequence variants: An overview of collapsing methods. Genetic Epidemiology, 2011;35(S1):S12-S17.

[51] Mantel N, Haenszel W. Statistical aspects of the analysis of data from retrospective studies of disease. Journal of the National Cancer Institute, 1959;22(4):719-748.

[52] Terada A, duVerle D, Tsuda K. Significant pattern mining with confounding variables. In Bailey J, Khan L, Washio T, et al., eds., Pacific-Asia Conference on Knowledge Discovery and Data Mining. Springer;2016, pp. 277-289.

[53] Terada A, Yamada R, Tsuda K, Sese J. Lamplink: Detection of statistically significant snp combinations from GWAS data. Bioinformatics, 2016;32(22):3513-3515.

[54] Yoshizoe K, Terada A, Tsuda K. Redesigning pattern mining algorithms for supercomputers, arXiv preprint available at https:/ / arxiv.org/pdf/1510.07787 .pdf. 2015.

[55] Llinares-Lopez F, Grimm D, Bodenham D, et al. Genome-wide detection of intervals of genetic heterogeneity associated with complex traits. Bioinformatics, 2015;31(12):i240-i249. 
[56] Benjamini Y, Hochberg Y. Controlling the false discovery rate: A practical and powerful approach to multiple testing. Journal of the Royal Statistical Society. Series B (Statistical Methodology), 1995;57(1):289-300.

[57] Taylor J, Tibshirani RJ. Statistical learning and selective inference. Proceedings of the National Academy of Sciences, 2015;112(25):7629-7634.

[58] Suzumura S, Nakagawa K, Umezu Y, Tsuda K, Takeuchi I. Selective inference for sparse high-order interaction models. In Precup D, Teh WY, eds., Proceedings of the 34th International Conference on Machine Learning, vol. 70. PMLR;2017, pp. 3338-3347. 